NISTIR 8217

\title{
Shape Analysis, Lebesgue Integration and Absolute Continuity Connections
}

Javier Bernal 
NISTIR 8217

\title{
Shape Analysis, Lebesgue Integration and Absolute Continuity Connections
}

\author{
Javier Bernal \\ Applied and Computational Mathematics Division \\ Information Technology Laboratory
}

This publication is available free of charge from:

https://doi.org/10.6028/NIST.IR.8217

July 2018

INCLUDES UPDATES AS OF 06-27-2019; SEE APPENDIX

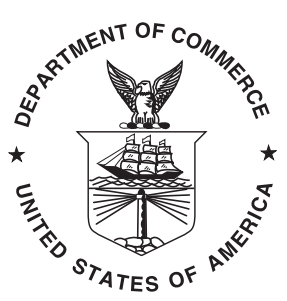

U.S. Department of Commerce Wilbur L. Ross, Jr., Secretary

National Institute of Standards and Technology Walter Copan, NIST Director and Undersecretary of Commerce for Standards and Technology 


\title{
Shape Analysis, Lebesgue Integration and Absolute Continuity Connections
}

\author{
Javier Bernal \\ National Institute of Standards and Technology, \\ Gaithersburg, MD 20899, USA
}

\begin{abstract}
As shape analysis of the form presented in Srivastava and Klassen's textbook "Functional and Shape Data Analysis" is intricately related to Lebesgue integration and absolute continuity, it is advantageous to have a good grasp of the latter two notions. Accordingly, in these notes we review basic concepts and results about Lebesgue integration and absolute continuity. In particular, we review fundamental results connecting them to each other and to the kind of shape analysis, or more generally, functional data analysis presented in the aforemetioned textbook, in the process shedding light on important aspects of all three notions. Many well-known results, especially most results about Lebesgue integration and some results about absolute continuity, are presented without proofs. However, a good number of results about absolute continuity and most results about functional data and shape analysis are presented with proofs. Actually, most missing proofs can be found in Royden's "Real Analysis" and Rudin's "Principles of Mathematical Analysis" as it is on these classic textbooks and Srivastava and Klassen's textbook that a good portion of these notes are based. However, if the proof of a result does not appear in the aforementioned textbooks, nor in some other known publication, or if all by itself it could be of value to the reader, an effort has been made to present it accordingly.
\end{abstract}




\section{Introduction}

The concepts of Lebesgue integration and absolute continuity play a major role in the theory of shape analysis or more generally in the theory of functional data analysis of the form presented in [23]. In fact, well-known connections between Lebesgue integration and absolute continuity are of great importance in the development of functional data and shape analysis of the kind in [23]. Accordingly, understanding functional data and shape analysis as presented in [23] requires understanding the basics of Lebesgue integration and absolute continuity, and the connections between them. It is the purpose of these notes to provide a way to do exactly that.

In Section 2, we review fundamental concepts and results about Lebesgue integration. Then, in Section 3, we review fundamental concepts and results about absolute continuity, some results connecting it to Lebesgue integration. Finally, in Section 4, we shed light on some important aspects of functional data and shape analysis of the type in Srivastava and Klassen's textbook [23], in the process illustrating its dependence on Lesbesgue integration, absolute continuity and the connections between them. Accordingly, without page numbers, a table of contents for these notes would be roughly as follows:

1. Introduction

2. Lebesgue Integration

Algebras of sets, Borel sets, Cantor set

Outer measure

Measurable sets, Lebesgue measure

Measurable functions, Step functions, Simple functions

The Riemann integral

The Lebesgue integral

The $L^{p}$ Spaces

3. Absolute Continuity and its Connections to Lebesgue Integration

4. Functional Data and Shape Analysis and its Connections to Lebesgue Integration and Absolute Continuity

Summary

Acknowledgements

References

Index of Terms

The material in these notes about Lebesgue integration and absolute continuity is mostly based on Royden's "Real Analysis" [16] and Rudin's "Principles of Mathematical Analysis" [18]. The fundamental ideas on functional 
data and shape analysis are mostly from Srivastava and Klassen's "Functional and Shape Data Analysis" [23]. An index of terms has been included at the end of the notes.

\section{Lebesgue Integration}

\section{Algebras of sets, Borel sets, Cantor set}

Definition 2.1: A collection $\mathcal{A}$ of subsets of a set $X$ is called an algebra on $X$ if for $A, B$ in $\mathcal{A}, A \cup B$ is in $\mathcal{A}$, and for $A$ in $\mathcal{A}, \tilde{A}=X \backslash A$ is in $\mathcal{A}$.

Observation 2.1: From De Morgan's laws if $\mathcal{A}$ is an algebra, then for $A, B$ in $\mathcal{A}, A \cap B$ is in $\mathcal{A}$.

Definition 2.2: An algebra $\mathcal{A}$ is called a $\boldsymbol{\sigma}$-algebra if the union of every countable collection of sets in $\mathcal{A}$ is in $\mathcal{A}$.

Observation 2.2: From De Morgan's laws if $\mathcal{A}$ is a $\sigma$-algebra, then the intersection of a countable collection of sets in $\mathcal{A}$ is in $\mathcal{A}$.

Definition 2.3: A set of real numbers $O$ is said to be open if for each $x \in O$ there is $\delta>0$ such that each number $y$ with $|x-y|<\delta$ belongs to $O$. A set of real numbers $F$ is said to be closed if its complement in $\mathbf{R}$ is open, i.e., $\mathbf{R} \backslash F$ is open, where $\mathbf{R}$ is the set of real numbers. The collection of Borel sets is the smallest $\sigma$-algebra on the set $\mathbf{R}$ of real numbers which contains all open sets of real numbers.

Observation 2.3: The collection of Borel sets contains in particular all closed sets, all open intervals, all countable unions of closed sets, all countable intersections of open sets, etc.

Proposition 2.1: Every open set of real numbers is the union of a countable collection of disjoint open intervals. Proof in [16].

Proposition 2.2 (Lindelöf): Given a collection $\mathcal{C}$ of open sets of real 
numbers, then there is a countable subcollection $\left\{O_{i}\right\}$ of $\mathcal{C}$ with

$$
\cup_{O \in \mathcal{C}} O=\cup_{i=1}^{\infty} O_{i}
$$

Proof in [16].

Definition 2.4: A set of real numbers $F$ is said to be compact if every open cover of $F$ contains a finite subcover, i.e., if $\mathcal{C}$ is a collection of open sets of real numbers such that $F \subseteq \cup_{O \in \mathcal{C}} O$, then there is a finite subcollection $\left\{O_{i}, i=1, \ldots, n\right\}$ of $\mathcal{C}$ with $F \subseteq \cup_{i=1}^{n} O_{i}$.

Proposition 2.3 (Heine-Borel): A set of real numbers $F$ is compact if and only if it is closed and bounded. Proof in [16] and [18].

Proposition 2.4: Given a collection $\mathcal{K}$ of closed sets of real numbers such that at least one of the sets is bounded and the intersection of every finite subcollection of $\mathcal{K}$ is nonempty, then $\cap_{F \in \mathcal{K}} F \neq \emptyset$. Proof in [16] and [18].

Definition 2.5: A number $x$ is said to be a limit point of a set of real numbers $E$ if every open set that contains $x$ contains $y \neq x, y$ in $E$.

Definition 2.6: A set of real numbers $E$ is said to be perfect if it is closed and if every number in $E$ is a limit point of $E$.

Proposition 2.5: A set is closed if and only if every limit point of the set is a point of the set. A nonempty perfect set is uncountable. Proofs in [18].

Corollary 2.1: Every interval is uncountable, thus the set of real numbers is uncountable.

Observation 2.4: Let $E_{1}$ be the union of the intervals $\left[0, \frac{1}{3}\right],\left[\frac{2}{3}, 1\right]$ that are obtained by removing the open middle third of the interval $[0,1]$. Let $E_{2}$ be the union of the intervals $\left[0, \frac{1}{9}\right],\left[\frac{2}{9}, \frac{3}{9}\right],\left[\frac{6}{9}, \frac{7}{9}\right],\left[\frac{8}{9}, 1\right]$ that are obtained by removing the open middle thirds of the intervals $\left[0, \frac{1}{3}\right]$ and $\left[\frac{2}{3}, 1\right]$. Continuing this way, a sequence of compact sets $E_{n}$ is obtained with $E_{n} \supset E_{n+1}$ for every positive integer $n$. The set $\cap_{n=1}^{\infty} E_{n}$, called the Cantor set, is compact, nonempty, perfect thus uncountable, and contains no interval. Proofs in [18]. 
Definition 2.7: The extended real numbers consist of the real numbers together with the two symbols $-\infty$ and $+\infty$. The definition of $<$ is extended by declaring that if $x$ is a real number, then $-\infty<x<\infty$. The operation $\infty-\infty$ is left undefined, the operation $0 \cdot( \pm \infty)$ is defined to be 0 , while other definitions are extended: If $x$ is a real number, then

$$
\begin{aligned}
& x+\infty=\infty, \quad x-\infty=-\infty, \quad x /+\infty=x /-\infty=0, \\
& x \cdot \infty=\infty, \quad x \cdot-\infty=-\infty \quad \text { if } x>0, \\
& x \cdot \infty=-\infty, \quad x \cdot-\infty=\infty \quad \text { if } x<0 .
\end{aligned}
$$

Finally

$\infty+\infty=\infty, \quad-\infty-\infty=-\infty, \quad \infty \cdot( \pm \infty)= \pm \infty, \quad-\infty \cdot( \pm \infty)=\mp \infty$.

\section{Outer measure}

Definition 2.8: Given a set $A$ of real numbers, the outer measure $m^{*} A$ of $A$ is the extended real number defined by

$$
m^{*} A=\inf _{A \subseteq \cup I_{n}} \sum l\left(I_{n}\right)
$$

where the $\left\{I_{n}\right\}$ are countable collections of open intervals that cover $A$, and $l\left(I_{n}\right)$ is the length of the interval $I_{n}$.

Observation 2.5: $m^{*}$ is a set function, $m^{*} \emptyset=0, m^{*} A \leq m^{*} B$ if $A \subseteq B$, and the outer measure of a set consisting of a single point is zero.

Proposition 2.6: $m^{*}(I)=l(I)$ if $I$ is an interval. Proof in [16].

Proposition 2.7: Countable subadditivity of $m^{*}: m^{*}\left(\cup A_{n}\right) \leq \sum m^{*} A_{n}$ for any countable collection $\left\{A_{n}\right\}$ of sets of real numbers. Proof in [16].

Corollary 2.2: $m^{*} A=0$ if $A$ is countable.

Observation 2.6: The Cantor set is an example of an uncountable set with outer measure zero. Proof in [18].

Proposition 2.8: $m^{*}$ is translation invariant, i.e., $m^{*}(E+y)=m^{*} E$ for any 
set $E$ of real numbers and any number $y$, where $E+y=\{x+y: x \in E\}$.

Proof: If $\left\{I_{n}\right\}$ is a countable collection of open intervals that covers $E$, then $\left\{I_{n}+y\right\}$ is a countable collection of open intervals that covers $E+y$. Since $l\left(I_{n}\right)=l\left(I_{n}+y\right)$ for each $n$, then $m^{*}(E+y) \leq m^{*} E$. Similarly, if $\left\{I_{n}\right\}$ is a countable collection of open intervals that covers $E+y$, then $\left\{I_{n}-y\right\}$ covers $E$, $l\left(I_{n}\right)=l\left(I_{n}-y\right)$, and therefore $m^{*} E \leq m^{*}(E+y)$. Thus, $m^{*} E=m^{*}(E+y)$.

Proposition 2.9: For any set $A$ of real numbers and any $\epsilon>0$, there is an open set $O$ with $A \subseteq O$ and $m^{*} O \leq m^{*} A+\epsilon\left(m^{*} O<m^{*} A+\epsilon\right.$ if $\left.m^{*} A<\infty\right)$. In addition, there is a set $G$ that is the intersection of a countable collection of open sets with $A \subseteq G$ and $m^{*} G=m^{*} A$.

Proof: From the definition of the outer measure there is a countable collection $\left\{I_{n}\right\}$ of open intervals that covers $A$ with $\sum l\left(I_{n}\right) \leq m^{*} A+\epsilon$. With $O=\cup I_{n}$, then $O$ is open, $A \subseteq O$, and $m^{*} O \leq \sum l\left(I_{n}\right) \leq m^{*} A+\epsilon$, which proves the first part. Now let $k>0$ be an integer. Then from the first part there is an open set $O_{k}, A \subseteq O_{k}$, with $m^{*} O_{k} \leq m^{*} A+\frac{1}{k}$. With $G=\cap_{k=1}^{\infty} O_{k}$, then for any integer $n>0$ we have $A \subseteq G \subseteq O_{n}$ and therefore $m^{*} A \leq m^{*} G \leq m^{*} O_{n} \leq m^{*} A+\frac{1}{n}$. Letting $n \rightarrow \infty$, then $m^{*} A \leq m^{*} G \leq m^{*} A$. Thus $m^{*} A=m^{*} G$, which proves the second part.

\section{Measurable sets, Lebesgue measure}

Definition 2.9 (Carathéodory's criterion): A set $E$ of real numbers is said to be (Lebesgue) measurable if for every set $A$ of real numbers, then

$$
m^{*} A=m^{*}(A \cap E)+m^{*}(A \cap \tilde{E}),
$$

$\tilde{E}$ the complement of $E$ in $\mathbf{R}$, i.e., $\tilde{E}=\mathbf{R} \backslash E, \mathbf{R}$ the set of real numbers.

Observation 2.7: Clearly $\tilde{E}$ is measurable if and only if $E$ is, and $\emptyset$ and the set $\mathbf{R}$ of real numbers are measurable. As for an example of a nonmeasurable set, a rather complex one is presented in [16]. Finally, note that it is always true that $m^{*} A \leq m^{*}(A \cap E)+m^{*}(A \cap \tilde{E})$, thus $E$ is measurable if and only if for every set $\bar{A}$ we have $m^{*} A \geq m^{*}(A \cap E)+m^{*}(A \cap \tilde{E})$. 
Proposition 2.10: If $m^{*} E=0$, then $E$ is measurable.

Proof: For any set $A$, since $A \supseteq A \cap \tilde{E}, m^{*} E=0$, and $E \supseteq A \cap E$, then $m^{*} A \geq m^{*}(A \cap \tilde{E})=m^{*}(A \cap \tilde{E})+m^{*}(E) \geq m^{*}(A \cap \tilde{E})+m^{*}(A \cap E)$.

Proposition 2.11: The collection of measurable sets is a $\sigma$-algebra on $\mathbf{R}$. Proof in [16].

Definition 2.10: The Lebesgue measure $m$ is the set function obtained by restricting the set function $m^{*}$ to the collection of (Lebesgue) measurable sets.

Proposition 2.12: Countable subadditivity of $m: m\left(\cup A_{n}\right) \leq \sum m A_{n}$ for any countable collection $\left\{A_{n}\right\}$ of measurable sets (Proposition 2.7).

Countable additivity of $m: m\left(\cup A_{n}\right)=\sum m A_{n}$ if the sets in $\left\{A_{n}\right\}$ as above are pairwise disjoint. Proof in [16].

Observation 2.8: It is in the proof of countable additivity of the Lebesgue measure $m$ that Carathéodory's criterion plays a major role. On the other hand, the importance of the countable additivity of $m$ is immediately apparent in the proofs of the two parts of the following very useful proposition.

Proposition 2.13 (Nested sequences of measurable sets Lemma):

1. Given a countable collection of measurable sets $\left\{E_{n}\right\}$ with $E_{n+1} \subseteq E_{n}$ for each $n, m E_{1}<\infty$, then $m\left(\cap_{i=1}^{\infty} E_{i}\right)=\lim _{n \rightarrow \infty} m E_{n}$.

2. Given a countable collection of (not necessarily measurable) sets $\left\{E_{n}\right\}$ with $E_{n+1} \supseteq E_{n}$ for each $n$, then $m^{*}\left(\cup_{i=1}^{\infty} E_{i}\right)=\lim _{n \rightarrow \infty} m^{*} E_{n}$.

Proof in [16] for the first part. In [14] for the second part using Proposition 2.9. As mentioned above, in the proofs of both parts the countable additivity of $m$ (Proposition 2.12) is used.

Proposition 2.14: Every Borel set is measurable. Proof in [16].

Observation 2.9: A rather complex example of a measurable set that is not Borel is presented in [4]. 
Proposition 2.15 (Equivalent conditions for a measurable set): Let $E$ be a set of real numbers. Then the following five conditions are equivalent:

i. $E$ is measurable.

ii. Given $\epsilon>0$, there is an open set $O, O \supseteq E$ with $m^{*}(O \backslash E)<\epsilon$.

iii. Given $\epsilon>0$, there is a closed set $F, F \subseteq E$ with $m^{*}(E \backslash F)<\epsilon$.

iv. There is a set $G$ that is the intersection of a countable collection of open sets, $G \supseteq E$ with $m^{*}(G \backslash E)=0$.

v. There is a set $F$ that is the union of a countable collection of closed sets, $F \subseteq E$ with $m^{*}(E \backslash F)=0$.

Proof: We only prove $\mathrm{i} \Leftrightarrow$ ii. Proofs of all cases in [17].

$\mathrm{i} \Rightarrow$ ii: $E$ is measurable.

Case 1: $m E<\infty$.

There exists $O$ open such that $E \subseteq O, m O<m E+\epsilon$ (Proposition 2.9). $m E<\infty$ then implies $m O-m E<\epsilon$. Accordingly,

$$
\begin{aligned}
\epsilon & >m(O \cap E)+m(O \cap \tilde{E})-m E \\
& =m E+m(O \backslash E)-m E \\
& =m(O \backslash E) .
\end{aligned}
$$

Case 2: $m E=\infty$.

For each integer $n>0$ let $E_{n}=E \cap((n-1, n] \cup(-n,-n+1])$. Then each $E_{n}$ is measurable, $m E_{n}<\infty$, and $E=\cup E_{n}$. From Case 1 above it follows that there is $O_{n}$ open such that $E_{n} \subseteq O_{n}$ and $m\left(O_{n} \backslash E_{n}\right)<\epsilon / 2^{n}$. With $O=\cup O_{n}$, then $O$ is open, $E \subseteq O$ and $O \backslash E \subseteq \cup\left(O_{n} \backslash E_{n}\right)$. Thus,

$$
m(O \backslash E) \leq m\left(\cup\left(O_{n} \backslash E_{n}\right)\right) \leq \sum m\left(O_{n} \backslash E_{n}\right)<\sum \epsilon / 2^{n}=\epsilon .
$$

ii $\Rightarrow$ i: Given $\epsilon>0$, there is an open set $O, O \supseteq E$ with $m^{*}(O \backslash E)<\epsilon$. $O$ open implies $O$ is measurable. Thus, for any set $A$, we have $m^{*} A=$ $m^{*}(A \cap O)+m^{*}(A \cap \tilde{O})$. Accordingly,

$$
\begin{aligned}
m^{*} A+\epsilon & >m^{*} A+m^{*}(O \cap \tilde{E}) \\
& =m^{*}(A \cap O)+m^{*}(A \cap \tilde{O})+m^{*}(O \cap \tilde{E}) \\
& \geq m^{*}(A \cap E)+m^{*}(A \cap \tilde{O} \cap \tilde{E})+m^{*}(O \cap \tilde{E} \cap A) \\
& =m^{*}(A \cap E)+m^{*}(A \cap \tilde{E}) .
\end{aligned}
$$

$\epsilon$ arbitrary implies $m^{*} A \geq m^{*}(A \cap E)+m^{*}(A \cap \tilde{E})$. Thus, $E$ is measurable. 
Corollary 2.3: Every (Lebesgue) measurable set is the union of a Borel set and a set of (Lebesgue) measure zero.

Proposition 2.16: The translate of a measurable set is measurable, i.e., if $E$ is a measurable set, then $E+y$ is measurable for any number $y$.

Proof: For any set $A$, setting $B=A-y, F=E+y$, and noting $(B \cap E)+y=$ $A \cap F,(B \cap \tilde{E})+y=A \cap \tilde{F}$, by Proposition 2.8, then

$$
m^{*} A=m^{*} B=m^{*}(B \cap E)+m^{*}(B \cap \tilde{E})=m^{*}(A \cap F)+m^{*}(A \cap \tilde{F}) .
$$

Thus, $F=E+y$ is measurable.

\section{Measurable functions, Step functions, Simple functions}

Definition 2.11: Let $f$ be an extended real-valued function defined on a (Lebesgue) measurable set. Then $f$ is said to be (Lebesgue) measurable if the set $\{x \mid f(x)>a\}$ is (Lebesgue) measurable for every real number $a$.

Proposition 2.17 (Equivalent conditions for a measurable function): Let $f$ be an extended real-valued function of measurable domain. Then the following conditions are equivalent:

i. $\{x \mid f(x)>a\}$ is measurable for every real number $a$.

ii. $\{x \mid f(x) \geq a\}$ is measurable for every real number $a$.

iii. $\{x \mid f(x)<a\}$ is measurable for every real number $a$.

iv. $\{x \mid f(x) \leq a\}$ is measurable for every real number $a$.

Proof in [16] and [18].

Observation 2.10: Conditions ii, iii, iv can be used instead of condition i to define a measurable function. We note that if a real-vaued function $f$ defined on a closed or open interval $I$ is continuous [18], then $f$ is measurable since the set in condition i is relatively open in $I$ [18]. Also the restriction of a measurable function to a measurable subset of its domain, is measurable.

Definition 2.12: Given real numbers $a, b, a<b$, and an integer $n>0$, by a partition or subdivision of $[a, b]$ we mean a finite set of points $P=\left\{\xi_{0}, \xi_{1}, \ldots, \xi_{n}\right\}$ with $a=\xi_{0}<\xi_{1}<\ldots<\xi_{n}=b$. A function $\psi:[a, b] \rightarrow$ 
$\mathbf{R}$ is called a step function (on $[a, b]$ ) if for an integer $n>0$, there are numbers $c_{i}, i=1, \ldots, n$, and a partition or subdivision $P=\left\{\xi_{0}, \xi_{1}, \ldots, \xi_{n}\right\}$ of $[a, b]$, such that $\psi(a)=\psi\left(\xi_{0}\right)=c_{1}, \psi(x)=c_{i}, \xi_{i-1}<x \leq \xi_{i}, i=1, \ldots, n$.

Definition 2.13: Given sets $A, X$ of real numbers, $A \subseteq X$, the characteristic function $\chi_{A}$ of $A$ on $X, \chi_{A}: X \rightarrow\{0,1\}$, is defined by

$$
\chi_{A}(x)= \begin{cases}1 & x \in A \\ 0 & x \in X \backslash A .\end{cases}
$$

Definition 2.14: Given a measurable set $E$, an integer $n>0$, and for $i=1, \ldots, n$, nonzero numbers $c_{i}$, measurable sets $E_{i} \subseteq E$, and characteristic functions $\chi_{E_{i}}$ of $E_{i}$ on $E$, a function $\varphi: E \rightarrow \mathbf{R}$ defined by $\varphi(x)=$ $\sum_{i=1}^{n} c_{i} \chi_{E_{i}}(x)$ for $x$ in $E$ is called a simple function on $E$.

Observation 2.11: Step functions are measurable and a function is simple if and only if it is measurable and assumes only a finite number of values. We note that the representation of a simple function $\varphi$ is not unique. However it does have a so-called canonical representation: for some integer $m>0, \varphi(x)=\sum_{i=1}^{m} a_{i} \chi_{A_{i}}(x)$ for $x$ in $E$, where $\left\{a_{1}, \ldots, a_{m}\right\}$ is the set of distinct nonzero values of $\varphi$, and $A_{i}=\left\{x \in E: \varphi(x)=a_{i}\right\}, i=1, \ldots, m$. This representation is characterized by the fact that the $a_{i}$ 's are distinct and nonzero, and the $A_{i}$ 's are pairwise disjoint.

Proposition 2.18: If $f$ is a measurable function, then the function $|f|$ is measurable. Proof in [18].

Proposition 2.19: Let $f$ and $g$ be measurable real-valued (not extended) functions defined on the same domain $X, c$ a constant, and $F$ a continuous real-valued function on $\mathbf{R}^{2}$. Then the function $h$ defined by $h(x)=$ $F(f(x), g(x)), x \in X$, is measurable. In particular $f+g, f g, c f, f+c, f-g$ are measurable; $f / g$ is measurable if $g \neq 0$ on $X$. Proofs in [16] and [18].

Observation 2.12: For extended real-valued measurable functions $f$ and $g$, the function $f g$ is still measurable. However in order for $f+g$ to be measurable, $f+g$ must be given the same value at points where it is undefined, unless these points form a set of measure zero in which case it makes no 
difference which values $f+g$ is given on the set.

Proposition 2.20: Let $\left\{f_{n}\right\}$ be a sequence of measurable functions defined on the same domain $X$ and let $N>0$ be an integer. The functions defined for each $x \in X$ by $\sup _{n \leq N} f_{n}(x), \sup _{n} f_{n}(x), \limsup _{n \rightarrow \infty} f_{n}(x), \inf _{n \leq N} f_{n}(x)$, $\inf _{n} f_{n}(x), \lim \inf _{n \rightarrow \infty} f_{n}(x)$, are all measurable. Proofs in [16] and [18].

Definition 2.15: Given a set of real numbers $X$, a property associated with points in $X$ is said to hold almost everywhere (a.e. for short) in $X$ if the set of points in $X$ where the property fails has measure zero.

Proposition 2.21: Let $f$ and $g$ be functions defined on the same measurable domain $X$. If $f$ is measurable and $f=g$ a.e., then $g$ is measurable.

Proof: Let $a$ be any real number. $f=g$ a.e. means the set $E=\{x: f(x) \neq$ $g(x)\}$ has measure zero. Thus $E$ and the set $\{x \in E: g(x)>a\} \subseteq E$ are measurable (Proposition 2.10). $X \backslash E$ must then be measurable and the set $\{x \in X \backslash E: f(x)>a\}$ is then measurable as $f$ is measurable. Thus, since

$$
\{x: g(x)>a\}=\{x \in X \backslash E: f(x)>a\} \cup\{x \in E: g(x)>a\}
$$

it follows that $\{x: g(x)>a\}$ is measurable and therefore $g$ is measurable.

Observation 2.13: The definition of a measurable function and 1 of Proposition 2.13 allow the following proposition to be true.

Proposition 2.22 (Egoroff's Theorem): Let $\left\{f_{n}\right\}$ be a sequence of measurable functions on a measurable set $E, m E<\infty$, that converge a.e. to a real-valued (not extended) function $f$ on $E$, i.e., there is a set $B \subseteq E$ such that $m B=0, f_{n} \rightarrow f$ pointwise on $E \backslash B$. Then for every $\delta>0$ there is a closed set $F \subseteq E$ such that $m(E \backslash F)<\delta$ and $f_{n} \rightarrow f$ uniformly on $F$.

Proof: Let $m>0$ be an integer. For each integer $n>0$ let

$$
G_{n}^{m}=\left\{x \in E:\left|f_{n}(x)-f(x)\right| \geq 1 / m\right\},
$$

and for each integer $N>0$ set

$$
E_{N}^{m}=\cup_{n=N}^{\infty} G_{n}^{m}=\left\{x \in E:\left|f_{n}(x)-f(x)\right| \geq 1 / m \text { for some } n \geq N\right\} .
$$


We note $f$ is measurable (Proposition 2.20 and Proposition 2.21). It follows that each $E_{N}^{m}$ is measurable and of finite measure, $E_{N+1}^{m} \subseteq E_{N}^{m}$, and for each $x \in E \backslash B$ there must be some $N$ for which $x \notin E_{N}^{m}$, since $f_{n}(x) \rightarrow f(x)$. Thus $\cap E_{N}^{m} \subseteq B$ and must therefore have measure zero. It follows then that $\lim _{N \rightarrow \infty} m E_{N}^{m}=0$ (1 of Proposition 2.13). Hence there exists $N$ such that

$$
m E_{N}^{m}=m\left(\left\{x \in E:\left|f_{n}(x)-f(x)\right| \geq 1 / m \text { for some } n \geq N\right\}\right)<\delta / 2^{m+1} .
$$

Letting $A^{m}=E_{N}^{m}$, then $A^{m}$ is measurable, $m A^{m}<\delta / 2^{m+1}$ and

$$
E \backslash A^{m}=\left\{x \in E:\left|f_{n}(x)-f(x)\right|<1 / m \text { for all } n \geq N\right\} .
$$

Now let $A=\cup_{m=1}^{\infty} A^{m}$. Then $m A \leq \sum_{m=1}^{\infty} m A^{m}<\sum_{m=1}^{\infty} \delta / 2^{m+1}=\delta / 2$.

Given $\epsilon>0$ choose integer $m>0$ with $1 / m<\epsilon$. For some $N$ and for all $x \in E \backslash A$ then $x \in E \backslash A^{m}$ and $\left|f_{n}(x)-f(x)\right|<1 / m<\epsilon$ for all $n \geq N$.

Thus, $f_{n} \rightarrow f$ uniformly on $E \backslash A$.

Finally, let $G=E \backslash A$. Clearly $G$ is measurable and $m(E \backslash G)=m A<\delta / 2$. Thus, there exists a closed set $F, F \subseteq G$ with $m(G \backslash F)<\delta / 2$ (Proposition 2.15). It then follows that $m(E \backslash F)=m(E \backslash G)+m(G \backslash F)<\delta / 2+\delta / 2=$ $\delta$ and $f_{n} \rightarrow f$ uniformly on $F$.

Proof also in [8] for spaces and measures more general than $\mathbf{R}$ and the Lebesgue measure.

Observation 2.14: The assumption $m E<\infty$ is necessary in the above proposition. To see this, let $E=\mathbf{R}$ and

$$
f_{n}(x)= \begin{cases}1 & x>n \\ 0 & x \leq n\end{cases}
$$

Clearly $f_{n} \rightarrow 0$ pointwise on $E$. However, for any integer $N>0,0<\epsilon<1$, the set $\left\{x \in E: f_{N}(x) \geq \epsilon\right\}=(N, \infty)$ is of infinite measure. Thus, the uniform convergence of $f_{n}$ to 0 as proposed in the proposition can not occur.

Definition 2.16: Given a function $f$ defined on a set $E$, the positive part $f^{+}$of $f$ and the negative part $f^{-}$of $f$ are the functions defined respectively by $f^{+}(x)=\max \{f(x), 0\}$, and $f^{-}(x)=\max \{-f(x), 0\}, x \in E$. 
Proposition 2.23 (Approximation of a measurable function by simple functions): Let $f$ be a real-valued (not extended) measurable function on a measurable set $E$. Then there exists a sequence of simple functions $\left\{s_{n}\right\}$ on $E$ such that $s_{n} \rightarrow f$ pointwise on $E$. Since $f=f^{+}-f^{-}$, and $f^{+}$and $f^{-}$ are measurable because $f$ is, then $\left\{s_{n}\right\}$ can be chosen so that $s_{n}=s_{n}^{u}-s_{n}^{l}$, where $s_{n}^{u}$ and $s_{n}^{l}$ are simple functions on $E$ such that $s_{n}^{u} \rightarrow f^{+}, s_{n}^{l} \rightarrow f^{-}$ pointwise on $E$, and $s_{n}^{u}$ and $s_{n}^{l}$ increase monotonically to $f^{+}$and $f^{-}$, respectively. If $f$ is bounded, then $\left\{s_{n}\right\}$ can also be chosen to converge uniformly to $f$ on $E$. Proof in [18].

Proposition 2.24 (Lusin's Theorem): Let $f$ be a real-valued (not extended) measurable function on a measurable set $E$. Then given $\epsilon>0$, there exists a closed set $F \subseteq E$ with $m(E \backslash F)<\epsilon$ such that $\left.f\right|_{F}$ is continuous.

Proof: First we prove the proposition for $f$ a simple function on $E$. Accordingly, for some integer $n>0$, assume $f(x)=\sum_{i=1}^{n} c_{i} \chi_{E_{i}}(x)$ for $x$ in $E, E_{i} \subseteq E$ for each $i$, the canonical representation of $f$. In addition let $E_{0}=E \backslash \cup_{i=1}^{n} E_{i}$. Clearly the $E_{i}$ 's are pairwise disjoint. Given $\epsilon>0$, since each $E_{i}$ is measurable there exists a closed set $F_{i} \subseteq E_{i}$ such that $m\left(E_{i} \backslash F_{i}\right)<\epsilon /(n+1)$, $i=0, \ldots, n$ (Proposition 2.15). Accordingly, $F=\cup_{i=0}^{n} F_{i}$ is closed, and

$$
m(E \backslash F)=m\left(\cup_{i=0}^{n} E_{i} \backslash \cup_{i=0}^{n} F_{i}\right)=m\left(\cup_{i=0}^{n}\left(E_{i} \backslash F_{i}\right)\right)=\sum_{i=0}^{n} m\left(E_{i} \backslash F_{i}\right)<\epsilon .
$$

Now, to show $\left.f\right|_{F}$ is continuous we show that if $\left\{x_{k}\right\}, x$ in $F$ are such that $x_{k} \rightarrow x$, then $f\left(x_{k}\right) \rightarrow f(x)$.

We note that for some unique $j, 0 \leq j \leq n$, it must be that $x \in F_{j}$. Thus, since $f$ is constant on $F_{j}$, it then suffices to show that for some integer $K>0$, $x_{k}$ is in $F_{j}$ for $k \geq K$. If this is not the case and since there is a finite number of $F_{i}$ 's then for some $l, 0 \leq l \leq n, l \neq j$, there is a subsequence $\left\{x_{k_{m}}\right\}$ of $\left\{x_{k}\right\}$ all contained in $F_{l}$. But then $x_{k_{m}} \rightarrow x$ so that $x$ is in $F_{l}$, a contradiction.

Now we prove the proposition for a general $f$.

Case 1: $m E<\infty$.

Let $s_{n}$ be simple functions such that $s_{n} \rightarrow f$ pointwise on $E$ (Proposition 2.23). Given $\epsilon>0$, as established above for simple functions on $E$, for each $n$ there exists a closed set $F_{n} \subseteq E$ with $m\left(E \backslash F_{n}\right)<\epsilon / 2^{n+1}$ such that $\left.s_{n}\right|_{F_{n}}$ is continuous. In addition, since $m E<\infty$, there exists a closed 
set $F_{0} \subseteq E$ such that $m\left(E \backslash F_{0}\right)<\epsilon / 2$ and $s_{n} \rightarrow f$ uniformly on $F_{0}$ (Proposition 2.22 (Egoroff's Theorem)).

Finally let $F=\cap_{n=0}^{\infty} F_{n}$. Then $F$ is closed and

$$
m(E \backslash F)=m\left(\cup_{n=0}^{\infty}\left(E \backslash F_{n}\right)\right) \leq \sum_{n=0}^{\infty} m\left(E \backslash F_{n}\right)<\sum_{n=0}^{\infty} \epsilon / 2^{n+1}=\epsilon .
$$

Since $\left.s_{n}\right|_{F_{n}}$ is continuous so must be $\left.s_{n}\right|_{F}$. And since $s_{n} \rightarrow f$ uniformly on $F \subseteq F_{0}$ then $\left.f\right|_{F}$ must be continuous (proof in [18]).

Case 2: $m E=\infty$.

For each integer $n>0$, let $\tilde{E}_{n}=(n-1, n] \cup(-n,-n+1]$ and $E_{n}=E \cap \tilde{E}_{n}$. Then each $E_{n}$ is measurable, $m E_{n}<\infty$, and $E=\cup_{n=1}^{\infty} E_{n}$. From Case 1 above, given $\epsilon>0$, it follows that there is a closed set $F_{n} \subseteq E_{n}$ with $m\left(E_{n}\right)$ $\left.F_{n}\right)<\epsilon / 2^{n}$ such that $\left.f\right|_{F_{n}}$ is continuous. Let $F=\cup_{n=1}^{\infty} F_{n}$. Then

$$
\begin{aligned}
m(E \backslash F) & =m\left(\cup_{n=1}^{\infty} E_{n} \backslash \cup_{n=1}^{\infty} F_{n}\right)=m\left(\cup_{n=1}^{\infty}\left(E_{n} \backslash F_{n}\right)\right) \\
& =\sum_{n=1}^{\infty} m\left(E_{n} \backslash F_{n}\right)<\sum_{n=1}^{\infty} \epsilon / 2^{n}=\epsilon .
\end{aligned}
$$

We show $F$ is closed. Let $x$ be a limit point of $F$ so that for $\left\{x_{k}\right\}$ in $F$, then $x_{k} \rightarrow x$. Clearly for some integer $j>0$ it must be that $x \in \tilde{E}_{j}$. It suffices to show that for some integer $K>0, x_{k}$ is in $F_{j}$ for $k \geq K$ so that $x$ is also in $F_{j} \subseteq F$. With $\tilde{E}_{n}=\emptyset$ for $n \leq 0$, from the definition of the $\tilde{E}_{n}$ 's a neighborhood of the point $x$ exists that does not intersect $\tilde{E}_{i}, i>j+1$ or $i<j-1$. Thus, with $F_{0}=\emptyset$, for $k$ large enough the points $x_{k}$ can only be in $F_{j-i}, F_{j}$ and $F_{j+1}$. If it is not the case that $K$ as described exists, then there must be a subsequence $\left\{x_{k_{m}}\right\}$ of $\left\{x_{k}\right\}$, all of it contained in either $F_{j-1}$ or $F_{j+1}$. But then $x_{k_{m}} \rightarrow x$ so that $x$ is in either $F_{j-1}$ or $F_{j+1}$, a contradiction. (Actually showing that $x$ is in the union of $F_{j-1}, F_{j}$ and $F_{j+1}$, would have sufficed). Now, to show $\left.f\right|_{F}$ is continuous we show that if $\left\{x_{k}\right\}, x$ in $F$ are such that $x_{k} \rightarrow x$, then $f\left(x_{k}\right) \rightarrow f(x)$.

We note that for some unique $j>0$, it must be that $x \in F_{j}$. Thus, since $f$ is continuous on $F_{j}$, it then suffices to show that for some integer $K>0, x_{k}$ is in $F_{j}$ for $k \geq K$. If this is not the case, again with $F_{0}=\emptyset$, an argument, similar to the one used above for proving $F$ is closed, can be used to get the same contradiction that $x$ is in either $F_{j-1}$ or $F_{j+1}$. 


\section{The Riemann integral}

Definition 2.17: Let $[a, b]$ be an interval and $f$ a bounded real-valued function defined on $[a, b]$. Given an integer $n>0$, and $P=\left\{\xi_{0}, \xi_{1}, \ldots, \xi_{n}\right\}$, a partition or subdivision of $[a, b]$ (Definition 2.12), for $i=1, \ldots, n$, we define

$$
\begin{aligned}
m_{i} & =\inf f(x), \quad \xi_{i-1} \leq x \leq \xi_{i}, \\
M_{i} & =\sup f(x), \xi_{i-1} \leq x \leq \xi_{i}, \text { and } \\
L(P, f) & =\sum_{i=1}^{n} m_{i}\left(\xi_{i}-\xi_{i-1}\right), \\
U(P, f) & =\sum_{i=1}^{n} M_{i}\left(\xi_{i}-\xi_{i-1}\right) .
\end{aligned}
$$

Then we define the lower Riemann integral and the upper Riemann integral of $f$ over $[a, b]$, respectively, by

$$
\begin{aligned}
& \mathcal{R} \underline{\int_{a}^{b}} f(x) d x=\sup _{P} L(P, f), \\
& \mathcal{R} \overline{\int_{a}^{b}} f(x) d x=\inf _{P} U(P, f),
\end{aligned}
$$

where the infimum and supremum are taken over all partitions $P$ of $[a, b]$. If the two are equal, $f$ is said to be Riemann integrable over $[a, b]$, and the common value is then called the Riemann integral of $f$ over $[a, b]$ and denoted by

$$
\mathcal{R} \int_{a}^{b} f(x) d x .
$$

Observation 2.15: Since $f$ is bounded, there exist numbers $m$ and $M$, such that $m \leq f(x) \leq M, x \in[a, b]$. Thus, for every partition $P$, it must be that $m(b-a) \leq L(P, f) \leq U(P, f) \leq M(b-a)$, so that the lower and upper Riemann integrals of $f$ over $[a, b]$ are finite numbers.

Proposition 2.25: $\mathcal{R} \underline{\int_{a}^{b}} f(x) d x \leq \mathcal{R} \int_{a}^{b} f(x) d x$. Proof in [18].

Observation 2.16: Let $\psi:[a, b] \rightarrow \mathbf{R}$ be a step function so that for an integer $n>0$, there are numbers $c_{i}, i=1, \ldots, n$, and a partition $P=$ $\left\{\xi_{0}, \xi_{1}, \ldots, \xi_{n}\right\}$ of $[a, b]$, such that $\psi(a)=\psi\left(\xi_{0}\right)=c_{1}, \psi(x)=c_{i}, \xi_{i-1}<x \leq \xi_{i}$, 
$i=1, \ldots, n$. Clearly $L(P, \psi)=U(P, \psi)$ and since $L(P, \psi) \leq \mathcal{R} \underline{\int_{a}^{b}} \psi(x) d x \leq$ $\mathcal{R} \int_{a}^{b} \psi(x) d x \leq U(P, \psi)$ (Proposition 2.25), it must be that $\psi$ is Riemann integrable over $[a, b]$ and $\mathcal{R} \int_{a}^{b} \psi(x) d x=\sum_{i=1}^{n} c_{i}\left(\xi_{i}-\xi_{i-1}\right)$.

From this it is then apparent that

$$
\begin{aligned}
& \mathcal{R} \underline{\mathcal{R}} \underline{\int_{a}^{b}} f(x) d x=\sup _{P} L(P, f)=\sup _{\psi \leq f} \mathcal{R} \int_{a}^{b} \psi(x) d x, \\
& \mathcal{\mathcal { R } \overline { \int } _ { a } ^ { b }} f(x) d x=\inf _{P} U(P, f)=\inf _{\psi \geq f} \mathcal{R} \int_{a}^{b} \psi(x) d x,
\end{aligned}
$$

where the $\psi$ 's are all possible step functions on $[a, b]$ satisfying the given conditions.

Definition 2.18: Given an interval $[a, b]$, let $P=\left\{\xi_{0}, \xi_{1}, \ldots, \xi_{n}\right\}$ be a partition of $[a, b]$. The number

$$
\mu(P)=\max _{i=1, \ldots, n}\left(\xi_{i}-\xi_{i-1}\right)
$$

is called the mesh of $P$.

Let $f$ be a real-valued function defined on $[a, b]$. Given a partition $P=$ $\left\{\xi_{0}, \xi_{1}, \ldots, \xi_{n}\right\}$ of $[a, b]$, a Riemann sum of $f$ with respect to $P$ is a sum of the form

$$
S(P, f)=\sum_{i=1}^{n} f\left(t_{i}\right)\left(\xi_{i}-\xi_{i-1}\right),
$$

where the choice of points $t_{1}, \ldots, t_{n}, \xi_{i-1} \leq t_{i} \leq \xi_{i}, i=1, \ldots, n$, is arbitrary. The Riemann sums of $f$ are said to converge to a finite number $I$ as $\mu(P) \rightarrow 0$, i.e.,

$$
I=\lim _{\mu(P) \rightarrow 0} S(P, f),
$$

if given $\epsilon>0$, there exists $\delta>0$ such that for every partition $P$ with mesh $\mu(P)<\delta$ it must be that

$$
|S(P, f)-I|<\epsilon
$$

(obviously for every choice of points $t_{1}, \ldots, t_{n}, \xi_{i-1} \leq t_{i} \leq \xi_{i}, i=1, \ldots, n$ ).

Proposition 2.26 (Riemann sums of $f$ that converge implies $f$ is bounded): If $\lim S(P, f)$ exists as $\mu(P) \rightarrow 0$, then $f$ is bounded on $[a, b]$. 
Proof in [13] and [15].

Proposition 2.27 (Riemann sums of $f$ converge if and only $f$ is Riemann integrable): Let $[a, b]$ be an interval and $f$ a bounded realvalued function defined on $[a, b]$. Then $f$ is Riemann integrable over $[a, b]$ if and only if

$$
I=\lim _{\mu(P) \rightarrow 0} S(P, f)
$$

exists. If this is the case, then $I$ equals $\mathcal{R} \int_{a}^{b} f(x) d x$. Proof in [18] and [25].

Observation 2.17: Given an interval $[a, b]$ and a set $A \subseteq[a, b]$, ideally the characteristic function $\chi_{A}$ of $A$ on $[a, b], \chi_{A}:[a, b] \rightarrow\{0,1\}$, defined by

$$
\chi_{A}(x)= \begin{cases}1 & x \in A \\ 0 & x \in[a, b] \backslash A\end{cases}
$$

should be (Riemann) integrable over $[a, b]$, especially if $A$ is measurable, and its integral over $[a, b]$ should equal the (outer) measure of $A$. However, if $A$ is the set of rational numbers in $[a, b]$, which is measurable with $m A=0$, we see that $\mathcal{R} \underline{\int_{a}^{b}} \chi_{A}(x) d x=0$ and $\mathcal{R} \int_{a}^{b} \chi_{A}(x) d x=b-a$, not the ideal situation.

\section{The Lebesgue integral}

Definition 2.19: Given a measurable set $E$, let $\varphi(x)=\sum_{i=1}^{m} a_{i} \chi_{A_{i}}(x)$ be the canonical representation of a simple function $\varphi$ on $E$, where for some integer $m>0,\left\{a_{1}, \ldots, a_{m}\right\}$ is the set of distinct nonzero values of $\varphi$, and $A_{i}=\left\{x \in E: \varphi(x)=a_{i}\right\}, i=1, \ldots, m$. We define the Lebesgue integral of $\varphi$ over $E$ as the extended real number

$$
\int_{E} \varphi(x) d x=\sum_{i=1}^{m} a_{i} m A_{i} .
$$

Observation 2.18: A consequence of the following two propositions is that if $\varphi(x)=\sum_{i=1}^{n} c_{i} \chi_{E_{i}}(x)$ is any representation of a simple function $\varphi$ on a measurable set $E$, then the Lebesgue integral of $\varphi$ over $E$ (Definition 2.19) can be computed directly from the representation, i.e., by computing $\sum_{i=1}^{n} c_{i} m E_{i}$.

Proposition 2.28: Let $\varphi(x)=\sum_{i=1}^{n} c_{i} \chi_{E_{i}}(x)$ be a representation of a simple 
function $\varphi$ on a measurable set $E$, with $E_{i} \cap E_{j}=\emptyset$ for $i \neq j$ (not necessarily the canonical representation of $\varphi$ ). Then

$$
\sum_{i=1}^{n} c_{i} m E_{i}=\int_{E} \varphi(x) d x .
$$

Proof in [16] for $E_{i}$ 's of finite measure. Same proof for the general case.

Proposition 2.29: Let $\varphi$ and $\psi$ be simple functions on a measurable set $E$. Then for any real numbers $a$ and $b$ we must have

$$
\int_{E}(a \varphi+b \psi)(x) d x=a \int_{E} \varphi(x) d x+b \int_{E} \psi(x) d x,
$$

and, if $\varphi \geq \psi$ a.e., then $\int_{E} \varphi(x) d x \geq \int_{E} \psi(d x)$.

Proof in [16] using Proposition 2.28 for $E_{i}$ 's of finite measure. Proof essentially the same for the general case.

Corollary 2.4: Let $\varphi(x)=\sum_{i=1}^{n} c_{i} \chi_{E_{i}}(x)$ be any representation of a simple function $\varphi$ on a measurable set $E$, the $E_{i}$ 's not necessarily pairwise disjoint. Then

$$
\sum_{i=1}^{n} c_{i} m E_{i}=\int_{E} \varphi(x) d x .
$$

Proof: Apply the first part of Proposition 2.29 to $\varphi(x)=\sum_{i=1}^{n} c_{i} \chi_{E_{i}}(x)$.

Definition 2.20: Given a measurable set $E$, let $f$ be a measurable nonnegative function on $E$. We define the Lebesgue integral of $f$ over $E$ as the extended real number

$$
\int_{E} f(x) d x=\sup _{\varphi \leq f} \int_{E} \varphi(x) d x
$$

where the $\varphi$ 's are all possible simple functions on $E$ satisfying the given condition.

Definition 2.21: Given a measurable set $E$, let $f$ be a measurable function on $E$. With $f^{+}$and $f^{-}$as the positive and negative parts of $f$ (Definition 2.16), we define the Lebesgue integral of $f$ over $E$ as the extended real number

$$
\int_{E} f(x) d x=\int_{E} f^{+}(x) d x-\int_{E} f^{-}(x) d x,
$$


if at least one of the integrals $\int_{E} f^{+}(x) d x, \int_{E} f^{-}(x) d x$ (Definition 2.20) is finite.

If $\int_{E} f(x) d x$ is finite, then $f$ is said to be Lebesgue integrable over $E$.

Proposition 2.30: Let $f$ and $g$ be Lebesgue integrable functions over a measurable set $E$, and $c$ a real number. Then

i. $c f$ is Lebesgue integrable over $E$ with $\int_{E} c f(x) d x=c \int_{E} f(x) d x$.

ii. $f+g$ is Lebesgue integrable over $E$ with

$$
\int_{E}(f+g)(x) d x=\int_{E} f(x) d x+\int_{E} g(x) d x .
$$

iii. If $f \leq g$ a.e., then $\int_{E} f(x) d x \leq \int_{E} g(x) d x$.

iv. If $A, B \subseteq E$ are disjoint measurable sets, then

$$
\int_{A \cup B} f(x) d x=\int_{A} f(x) d x+\int_{B} f(x) d x .
$$

Proofs in [16] and [18].

Proposition 2.31: A measurable function $f$ is Lebesgue integrable over $E$ if and only if $|f|$ is Lebesgue integrable over $E$, in which case

$$
\left|\int_{E} f(x) d x\right| \leq \int_{E}|f(x)| d x .
$$

Also, if $0 \leq f \leq g$ on $E$ and $g$ is Lebesgue integrable over $E$, then $f$ is Lebesgue integrable over $E$. In particular, if $|f| \leq g$ and $g$ is Lebesgue integrable, then $|f|$, and therefore $f$, is Lesbegue integrable over $E$.

Proof: The first part follows from $f=f^{+}-f^{-},|f|=f^{+}+f^{-}$, and iv of Proposition 2.30. The inequality from $f \leq|f|,-f \leq|f|$, and i and iii of Proposition 2.30. The rest from Definition 2.20.

Observation 2.19: Let $f$ be a measurable function on a measurable set $E$ with $m E$ finite, and let $a, b$ be real numbers such that $a \leq f(x) \leq b$ for $x \in E$. By looking at $\int_{E} f^{+}(x) d x$ and $\int_{E} f^{-}(x) d x$ for the different possible signs of $a$ and $b$, then it is evident that $a m E \leq \int_{E} f(x) d x \leq b m E$. Accordingly, if $f$ is a measurable and bounded function on a measurable set $E$ with $m E$ finite, since then for some $M>0,-M \leq f(x) \leq M$ for $x \in E$, it must be 
that $-M m E \leq \int_{E} f(x) d x \leq M m E$, and therefore $f$ is Lebesgue integrable. However, there is more to this situation as the following proposition shows.

Proposition 2.32 (Integrable equivalent to measurable): Let $f$ be a bounded function defined on a measurable set $E$ with $m E$ finite. Let

$$
L(f)=\sup _{\varphi \leq f} \int_{E} \varphi(x) d x, \quad U(f)=\inf _{\varphi \geq f} \int_{E} \varphi(x) d x
$$

where the $\varphi$ 's are all possible simple functions on $E$ satisfying the given conditions. Then $L(f)=U(f)$ if and only if $f$ is measurable. Whenever $L(f)=U(f)$ then $f$ is Lebesgue integrable and $\int_{E} f(x) d x=L(f)=U(f)$. Proof in [16].

Proposition 2.33 (Riemann integrable implies Lebesgue integrable): Let $f$ be a bounded function on interval $[a, b]$. If $f$ is Riemann integrable over $[a, b]$, then $f$ is measurable and Lebesgue integrable over $[a, b]$ with

$$
\int_{[a, b]} f(x) d x=\mathcal{R} \int_{a}^{b} f(x) d x .
$$

Proof: Since step functions are simple functions, then

$$
\sup _{\psi \leq f} \mathcal{R} \int_{a}^{b} \psi(x) d x \leq \sup _{\varphi \leq f} \int_{[a, b]} \varphi(x) d x \leq \inf _{\varphi \geq f} \int_{[a, b]} \varphi(x) d x \leq \inf _{\psi \geq f} \mathcal{R} \int_{a}^{b} \psi(x) d x
$$

where the $\psi$ 's and the $\varphi$ 's are all possible step functions and simple functions on $[a, b]$, respectively, satisfying the given conditions. Since $f$ is Riemann integrable over $[a, b]$, then all the inequalities above are equalities so that $f$ must be measurable and Lebesgue integrable over $[a, b]$ with

$$
\int_{[a, b]} f(x) d x=\sup _{\varphi \leq f} \int_{[a, b]} \varphi(x) d x=\inf _{\varphi \geq f} \int_{[a, b]} \varphi(x) d x=\mathcal{R} \int_{a}^{b} f(x) d x
$$

by Proposition 2.32 .

Proposition 2.34: Let $f$ be a measurable function on a measurable set $E$.

i. If $f \geq 0$ on $E$ and $\int_{E} f(x) d x=0$, then $f=0$ a.e. on $E$.

ii. If $f$ is Lebesgue integrable over $E$, then $f$ is finite a.e. on $E$. 
Proof: For each integer $n>0$ let $E_{n}=\{x \in E: f(x)>1 / n\}$.

For each $n$, we note $m E_{n}=0$ or else $\int_{E} f(x) d x>0$.

Let $A=\{x \in E: f(x) \neq 0\}$. Then $A=\cup_{n=1}^{\infty} E_{n}$. Thus, $m A=m\left(\cup_{n=1}^{\infty} E_{n}\right) \leq$ $\sum_{n=1}^{\infty} m\left(E_{n}\right)=0$ so that $f=0$ a.e. on $E$, which proves i.

In order to prove ii, for each integer $n>0$ let $E_{n}=\{x \in E:|f(x)| \geq n\}$.

Then $n \cdot m E_{n} \leq \int_{E_{n}}|f(x)| d x \leq \int_{E}|f(x)| d x=C$, so that $m E_{n} \leq C / n$.

Let $A=\{x \in E:|f(x)|=\infty\}$. Then $A=\cap_{n=1}^{\infty} E_{n}$. Since for each $n$, $A \subseteq E_{n}$, then $m A \leq m E_{n} \leq C / n$ so that $m A=0$, which proves ii.

Proposition 2.35 (Lebesgue's criterion for Riemann integrability): Let $f$ be a bounded function on $[a, b]$. Then $f$ is Riemann integrable over $[a, b]$ if and only if $f$ is continuous a.e. on $[a, b]$. Proof in [18]. It involves Proposition 2.33 and i of Proposition 2.34.

Observation 2.20: Function $\chi_{A}$ in Observation 2.17 with $A$ equal to the set of rational numbers fails the continuity hypothesis of Proposition 2.35 and thus it is not Riemann integrable over $[a, b]$ as observed there. Actually, it can be easily shown to be nowhere continuous on $[a, b]$.

Proposition 2.36 (Countable additivity of the Lebesgue integral): Let $\left\{E_{n}\right\}$ be a countable collection of pairwise disjoint measurable sets. Let $E=\cup_{i=1}^{\infty} E_{n}$, and let $f$ be a measurable function on $E$. Assume either $f \geq 0$ on $E$ or $f$ is Lebesgue integrable over $E$. Then

$$
\int_{E} f(x) d x=\sum_{i=1}^{\infty} \int_{E_{i}} f(x) d x .
$$

Proof in [18].

Observation 2.21: If $f$ is a measurable function on a set $E$ with $m E=0$, then $\int_{E} f(x) d x=0$. Also, if sets $A, E$ are measurable with $A \subseteq E$, and $f$ is Lebesgue integrable over $E$, then it is Lebesgue integrable over $A$. From all this then, if $f$ and $g$ are functions on a measurable set $E, f=g$ a.e. on $E, f$ Lebesgue integrable over $E$, then so is $g$ and $\int_{E} g(x) d x=\int_{E} f(x) d x$. Finally, we note that since integrals over sets of measure zero are zero, throughout these notes, if sets $F$ and $E$ are measurable with $F \subseteq E, m F=0$, and $f$ is a function on $E \backslash F$, possibly not defined on part or all of $F$, $f$ Lebesgue integrable over $E \backslash F$, we say $f$ is Lebesgue integrable over $E$ 
with $\int_{E} f(x) d x=\int_{E \backslash F} f(x) d x$. This makes sense as it is always possible to define $f$ arbitrarily for points in $F$ so that then $f$ is defined on all of $E$ and $\int_{E} f(x) d x=\int_{E \backslash F} f(x) d x+\int_{F} f(x) d x=\int_{E \backslash F} f(x) d x+0=\int_{E \backslash F} f(x) d x$.

Proposition 2.37 (Lebesgue's Monotone Convergence Theorem): Let $\left\{f_{n}\right\}$ be an increasing sequence of nonnegative measurable functions on a measurable set $E$. Let $f$ be defined by $f(x)=\lim _{n \rightarrow \infty} f_{n}(x)$ for $x \in E$. Then

$$
\int_{E} f(x) d x=\lim _{n \rightarrow \infty} \int_{E} f_{n}(x) d x .
$$

Proof in [16] and [18]. It involves Proposition 2.13.

Corollary 2.5: Let $\left\{f_{n}\right\}$ be a sequence of nonnegative measurable functions on a measurable set $E$. Let $f$ be defined by $f(x)=\sum_{n=1}^{\infty} f_{n}(x)$ for $x \in E$. Then

$$
\int_{E} f(x) d x=\sum_{n=1}^{\infty} \int_{E} f_{n}(x) d x .
$$

Proof: $\left\{h_{n}\right\}$ defined by $h_{n}(x)=\sum_{i=1}^{n} f_{i}(x)$ for $x \in E$ is an increasing sequence of nonnegative measurable functions on $E$.

Observation 2.22: Proposition 2.36 can now be proved more easily. It suffices to prove it for $f \geq 0$ on $E$. Let $f_{n}(x)=f(x) \cdot \chi_{E_{n}}(x)$ for $x \in E$. Then $f(x)=\sum_{n=1}^{\infty} f_{n}(x)$ for $x \in E$ and the result follows from Corollary 2.5.

Observation 2.23: The following proposition says that if a nonnegative function is Lebesgue integrable over a measurable set, then the Lebesgue integral of the function over a measurable subset of the set is arbitrarily small if the measure of the subset is small enough. Later we will see that it can be used to show that every indefinite integral is absolutely continuous (indefinite integrals and absolute continuity defined in the next section).

Proposition 2.38 (Absolute continuity of the Lebesgue integral): Let $f$ be a nonnegative Lebesgue integrable function over a measurable set $E$. Then given $\epsilon>0$ there is $\delta>0$ such that for each measurable set $A \subseteq E$ with $m A<\delta$, then $\int_{A} f(x) d x<\epsilon$. Proof in [16]. It involves Lebesgue's Monotone Convergence Theorem (Proposition 2.37). 
Proposition 2.39 (Fatou's Lemma): Let $\left\{f_{n}\right\}$ be a sequence of nonnegative measurable functions on a measurable set $E$. Let $f$ be defined by $f(x)=\liminf _{n \rightarrow \infty} f_{n}(x)$ for $x \in E$. Then

$$
\int_{E} f(x) d x \leq \liminf _{n \rightarrow \infty} \int_{E} f_{n}(x) d x .
$$

Proof in [16] and [18]. It involves Proposition 2.37.

Proposition 2.40 (Lebesgue's Dominated Convergence Theorem): Let $\left\{f_{n}\right\}$ be a sequence of measurable functions on a measurable set $E$ such that there is a function $f$ on $E$ with $f_{n} \rightarrow f$ pointwise a.e. on $E$. If there is a function $g$ that is Lebesgue integrable over $E$ such that $\left|f_{n}\right| \leq g$ on $E$ for all $n$, then

$$
\int_{E} f(x) d x=\lim _{n \rightarrow \infty} \int_{E} f_{n}(x) d x .
$$

Proof in [16] and [18]. It involves Proposition 2.39.

Corollary 2.6 (Bounded Convergence Theorem): Let $\left\{f_{n}\right\}$ be a sequence of measurable functions on a measurable set $E$ of finite measure such that there is a function $f$ on $E$ with $f_{n} \rightarrow f$ pointwise a.e. on $E$. If there is a real number $M$ such that $\left|f_{n}\right| \leq M$ on $E$ for all $n$, then

$$
\int_{E} f(x) d x=\lim _{n \rightarrow \infty} \int_{E} f_{n}(x) d x
$$

\section{The $L^{p}$ Spaces}

Definition 2.22: Given a real number $p>0$, the $L^{p}[0,1]$ or $L^{p}$ space is the space of measurable functions on $[0,1]$ satisfying: the $p$-th power of the absolute value of each function in the space is Lebesgue integrable over $[0,1]$. Thus, a measurable function $f$ on $[0,1]$ is in $L^{p}$ (the $L^{p}$ space) if and only if

$$
\int_{[0,1]}|f(x)|^{p} d x<\infty
$$

Writing $\int_{0}^{1}|f(x)|^{p} d x$ instead of $\int_{[0,1]}|f(x)|^{p} d x$ for $f$ in $L^{p}$, we define

$$
\|f\|_{p}=\left\{\int_{0}^{1}|f(x)|^{p} d x\right\}^{1 / p}
$$


and call $\|\cdot\|_{p}$ the $L^{p}$ norm, and $\|f\|_{p}$ the $L^{p}$ norm of $f$.

Finally, the $L^{\infty}[0,1]$ or $L^{\infty}$ space is the space of measurable functions on $[0,1]$ satisfying: each function in the space is bounded on $[0,1]$ except possibly on a set of measure zero. Thus, a measurable function $f$ on $[0,1]$ is in $L^{\infty}$ (the $L^{\infty}$ space) if and only if the essential supremum of $|f|$ on $[0,1]$ is finite, i.e.,

$$
\text { ess } \sup |f(t)|=\inf \{M: m(\{t:|f(t)|>M\})=0\}<\infty .
$$

We also note ess $\sup |f(t)|=\inf \left\{\sup _{t \in[0,1]}|g(t)|: g=f\right.$ a.e. $\}$. Defining

$$
\|f\|_{\infty}=\operatorname{ess} \sup |f(t)|
$$

we call $\|\cdot\|_{\infty}$ the $L^{\infty}$ norm, and $\|f\|_{\infty}$ the $L^{\infty}$ norm of $f$.

Observation 2.24: In the definition of the $L^{p}$ spaces, the interval $[0,1]$ was chosen for simplicity. Given a real number $p>0$, if $f \in L^{p}$, then clearly $c f \in L^{p}$ for any real number $c$. In addition, if $f, g \in L^{p}$, since $|f+g|^{p} \leq 2^{p}\left(|f|^{p}+|g|^{p}\right)$, then $f+g \in L^{p}$. Thus, $L^{p}$ is a linear space and so is $L^{\infty}$.

Given $f$ in $L^{p}, 0<p \leq \infty$, then the $L^{p}$ norm of $f$, i.e., $\|f\|_{p}$ (Definition 2.22), equals zero if and only if $f=0$ a.e. on $[0,1]$. Accordingly, we think of the elements of $L^{p}$ as equivalent classes of functions, each class composed of functions that are equal to one another a.e. on $[0,1]$, and as noted in Observation 2.21, some functions undefined on subsets of $[0,1]$ of measure zero. Thus, assuming there is no distinction between two functions in the same equivalence class, we note that given $p, 1 \leq p \leq \infty$, then the $L^{p}$ norm $\|\cdot\|_{p}$ is indeed a norm since clearly $\|c f\|_{p}=c\|f\|_{p}$ for any real number $c$, and as will be seen below, if $f, g \in L^{p}$, then $\|f+g\|_{p} \leq\|f\|_{p}+\|g\|_{p}$.

Proposition 2.41 (Hölder's inequality): Given $p, q, 1 \leq p, q \leq \infty$, with $1 / p+1 / q=1$, if $f \in L^{p}$ and $g \in L^{q}$, then $f \cdot g \in L^{1}$ and

$$
\int_{0}^{1}|(f \cdot g)(x)| d x \leq\|f\|_{p} \cdot\|g\|_{q},
$$

with equality for $p, q, 1<p, q<\infty$ if and only if $\alpha|f|^{p}=\beta|g|^{q}$ a.e. for nonzero constants $\alpha$ and $\beta$. Proof in [16], [19]. Proof in [18] for $p=q=2$.

Proposition 2.42 (Minkowski's inequality): Given $p, 1 \leq p \leq \infty$, if 
$f, g \in L^{p}$, then $f+g \in L^{p}$ and

$$
\|f+g\|_{p} \leq\|f\|_{p}+\|g\|_{p} .
$$

Proof in [16], [19]. Proof in [18] for $p=q=2$.

Observation 2.25: For $p=q=2$, Hölder's inequality becomes Schwarz's inequality:

$$
\int_{0}^{1}|(f \cdot g)(x)| d x \leq\|f\|_{2} \cdot\|g\|_{2}=\left\{\int_{0}^{1}|f(x)|^{2} d x\right\}^{1 / 2} \cdot\left\{\int_{0}^{1}|g(x)|^{2} d x\right\}^{1 / 2} .
$$

Note all of the above inequalities (Hölder's, Minkowski's, Schwarz's), in which all integrations are over $[0,1]$, can be generalized by integrating everywhere over a measurable set instead. Proof in [19].

Definition 2.23: Given a norm $\|\cdot\|$ on a linear space $X$, we say $X$ is a normed linear space with norm $\|\cdot\|$. We say this especially if among all the possible norms that can be defined on $X$, our current intent is to associate $X$ exclusively with $\|\cdot\|$.

A sequence $\left\{x_{n}\right\}$ in a normed linear space with norm $\|\cdot\|$ is said to converge in norm to an element $x$ in the space if, given $\epsilon>0$, there is an integer $N>0$ such that for $n \geq N$, then $\left\|x_{n}-x\right\|<\epsilon$.

A sequence $\left\{x_{n}\right\}$ in a normed linear space with norm $\|\cdot\|$ is said to be a Cauchy sequence if, given $\epsilon$, there is an integer $N>0$ such that for $n, m \geq N$, then $\left\|x_{n}-x_{m}\right\|<\epsilon$.

A normed linear space with norm $\|\cdot\|$ is called complete if every Cauchy sequence in the space converges in norm to an element of the space.

Proposition 2.43 (Riesz-Fischer): Given $p, 1 \leq p \leq \infty$, then $L^{p}$ is complete. Moreover, given $\left\{f_{n}\right\} \rightarrow f$ in $L^{p}$, then a subsequence of $\left\{f_{n}\right\}$ converges pointwise to $f$ a.e. on $[0,1]$. Proof of first part in [16], [17]. It involves Proposition 2.37 (Lebesgue's Monotone Convergence Theorem), Proposition 2.39 (Fatou's Lemma), Proposition 2.40 (Lebesgue's Dominated Convergence Theorem) and ii of Proposition 2.34. Proof of last part in [17].

Proposition 2.44 (Density of simple and step functions in $L^{p}$ space): Given $p, 1 \leq p \leq \infty$, then the subspace of simple functions on $[0,1]$ in $L^{p}$ is dense in $L^{p}$. Given $p, 1 \leq p<\infty$, then the subspace of step functions on $[0,1]$ is dense in $L^{p}$. Proof in [17]. 


\section{Absolute Continuity and its Connections to Lebesgue Integration}

Definition 3.1: Let $f$ be a real-valued function defined on an interval $[a, b]$. Given $x \in[a, b]$, if for some finite number $I$,

$$
I=\lim _{t \rightarrow x} \frac{f(t)-f(x)}{t-x}, \quad a<t<b, \quad t \neq x,
$$

then $f$ is said to be differentiable at $x$; a number $f^{\prime}(x)$ is defined and said to exist by setting $f^{\prime}(x)$ equal to $I$; and $f^{\prime}$ is said to exist at $x$. Accordingly, $f^{\prime}$ is a function associated with $f$, called the derivative of $f$, whose domain of definition is the set of points $x$ at which $f^{\prime}$ exists. If $f^{\prime}$ exists at every point of a set $E \subseteq[a, b]$, we say $f$ is differentiable on $E$ or $f^{\prime}$ exists on $E$.

Note that given $x \in[a, b]$, if the limit defining $I$ above equals $\infty$ or $-\infty$ then the convention here is to say that $f$ is not differentiable at $x$.

Proposition 3.1 (Fundamental Theorem of calculus I): Let $f$ be Riemann integrable over an interval $[a, b]$. If there is a function $F$ differentiable on $[a, b]$ such that $F^{\prime}=f$ on $[a, b]$, then

$$
\mathcal{R} \int_{a}^{b} f(x) d x=F(b)-F(a) .
$$

Proof in [1] and [18].

Proposition 3.2 (Fundamental Theorem of calculus II): Let $f$ be Riemann integrable over an interval $[a, b]$. Define a function $F$ by

$$
F(x)=\mathcal{R} \int_{a}^{x} f(t) d t, \quad x \in[a, b] .
$$

Then $F$ is continuous on $[a, b]$, and if $f$ is continuous at $x \in[a, b]$, then $F$ is differentiable at $x$ with $F^{\prime}(x)=f(x)$. Proof in [1] and [18].

Corollary 3.1 (Differentiability of the Riemann integral - Fundamental Theorem of calculus for continuous functions):

i. If $f$ is Riemann integrable over $[a, b]$ and $F(x)=\mathcal{R} \int_{a}^{x} f(t) d t, x \in[a, b]$, then $F^{\prime}=f$ a.e. on $[a, b]$.

ii. If $f$ is continuous on $[a, b]$, then there is a differentiable function $F$ on $[a, b]$ 
such that $F^{\prime}=f$ on $[a, b]$, and $\mathcal{R} \int_{a}^{x} f(t) d t=F(x)$. If $G$ is any differentiable function on $[a, b]$ such that $G^{\prime}=f$ on $[a, b]$, then $G-F=C, C$ a constant, and $\mathcal{R} \int_{a}^{x} f(t) d t=G(x)-G(a), G(a)=C$.

Proof: i follows from Proposition 3.2 and Proposition 2.35 (Lebesgue's criterion). First part of ii from Proposition 3.2. Proof in [18] that $G^{\prime}-F^{\prime}=0$ on $[a, b]$ implies $G-F=C$ on $[a, b], C$ a constant. $\mathcal{R} \int_{a}^{x} f(t) d t=G(x)-G(a)$ from Proposition 3.1. Clearly $G(a)=C$ as $\mathcal{R} \int_{a}^{x} f(t) d t=F(x)$.

Definition 3.2: Let $f$ be a real-valued function defined on an interval $[a, b]$. Given $x \in[a, b]$, if for some finite number $I, I=\lim _{t \rightarrow x} f(t), a \leq t \leq x$, then a number $f\left(x^{-}\right)$called the left-hand limit of $f$ at $x$ is defined by setting $f\left(x^{-}\right)$equal to $I$. Similarly, if for some finite number $I, I=\lim _{t \rightarrow x} f(t)$, $x \leq t \leq b$, then a number $f\left(x^{+}\right)$called the right-hand limit of $f$ at $x$ is defined by setting $f\left(x^{+}\right)$equal to $I$.

Observation 3.1: A function $f$ is continuous at $x \in[a, b]$ if and only if $f\left(x^{-}\right)$and $f\left(x^{+}\right)$exist and $f(x)=f\left(x^{-}\right)=f\left(x^{+}\right)$.

Proposition 3.3 (Monotonic functions: continuity): Let $f$ be a monotonic real-valued function on an interval $[a, b]$. Then $f\left(x^{-}\right)$and $f\left(x^{+}\right)$exist for every point $x \in[a, b]$, and the set of points of $[a, b]$ at which $f$ is discontinuous is at most countable. Proof in [18].

Corollary 3.2 (Monotonic surjective $f$ implies $f$ is continuous): If $f$ is monotonic from $[a, b]$ onto $[c, d]$, then $f$ is continuous on $[a, b]$.

Proof: Assume $f$ is discontinuous at $x \in[a, b]$. Since $f\left(x^{-}\right)$and $f\left(x^{+}\right)$exist from Proposition 3.3, it must be that $f\left(x^{-}\right) \neq f\left(x^{+}\right)$so that $y$ exists in $[c, d]$ between $f\left(x^{-}\right)$and $f\left(x^{+}\right), y \neq f(x)$. But then $y$ can not be in the range of $f$ as $f$ is monotonic, which contradicts that the range of $f$ is all of $[c, d]$.

Observation 3.2: A function $f$ is described below from $[0,1]$ into $[0,1]$ that is strictly increasing on $[0,1]$, discontinuous at each rational number in $(0,1]$, continuous at each irrational number in $[0,1]$ and at zero, $f(0)=0, f(1)=1$. 
Let $\left\{r_{n}\right\}_{n=1}^{\infty}$ be an enumeration of the rational numbers in $(0,1]$.

Given $x \in(0,1]$, let $R(x)=\left\{n: r_{n} \leq x\right\}$, and set $R(0)=\emptyset$.

Define $f:[0,1] \rightarrow[0,1]$ by $f(0)=0$ and

$$
f(x)=\sum_{n \in R(x)} 1 / 2^{n}, \quad x \in(0,1] .
$$

Given $x, x^{\prime} \in[0,1], x<x^{\prime}$, then $R(x) \subseteq R\left(x^{\prime}\right)$, and since there is a rational number $r$ such that $x<r<x^{\prime}$, then $R(x) \neq R\left(x^{\prime}\right)$. Thus, it must be that $f(x)<f\left(x^{\prime}\right)$ so that $f$ is strictly increasing on $[0,1]$.

Since $R(1)$ includes every $n$ then $f(1)=1$.

Let $x$ be a rational number in $(0,1]$. We show $f$ is discontinuous at $x$. For some integer $k>0, x=r_{k}$. Thus, $k \in R(x)$ but $k \notin R\left(x^{\prime}\right)$ for every $x^{\prime} \in[0,1], x^{\prime}<x . R\left(x^{\prime}\right) \subseteq R(x)$ then implies $f(x)-f\left(x^{\prime}\right)>1 / 2^{k}$.

Thus, $f$ is discontinuous at $x$ (a rational number in $(0,1]$ ).

Let $x$ be an irrational number in $[0,1]$. We show $f$ is continuous at $x$. Given $\epsilon>0$, choose integer $N>0$ such that $1 / 2^{N}<\epsilon$, and let

$$
\delta=\min _{n \leq N}\left|x-r_{n}\right|
$$

Given $x^{\prime} \in[0,1], x^{\prime}<x,\left|x-x^{\prime}\right|<\delta$, then $R\left(x^{\prime}\right) \subseteq R(x)$, and if $n \in$ $R(x) \backslash R\left(x^{\prime}\right)$, it must be that $x^{\prime}<r_{n}<x$ so that $\left|x-r_{n}\right|<\delta$ and thus $n>N$. Accordingly, $f(x)-f\left(x^{\prime}\right) \leq \sum_{n=N+1}^{\infty} 1 / 2^{n}=1 / 2^{N}<\epsilon$.

Finally, given $x^{\prime} \in[0,1], x^{\prime}>x,\left|x^{\prime}-x\right|<\delta$, then $R(x) \subseteq R\left(x^{\prime}\right)$, and if $n \in R\left(x^{\prime}\right) \backslash R(x)$, it must be that $x<r_{n} \leq x^{\prime}$ so that $\left|x-r_{n}\right|<\delta$ and thus $n>N$. Accordingly, $f\left(x^{\prime}\right)-f(x) \leq \sum_{n=N+1}^{\infty} 1 / 2^{n}=1 / 2^{N}<\epsilon$.

Thus, $f$ is continuous at $x$ (an irrational number in $[0,1]$ ) and at zero by an argument similar to the one just used for the case $x^{\prime}>x$.

Proposition 3.4 (Monotonic functions: differentiability): Let $f$ be a monotonic real-valued function on an interval $[a, b]$. Then $f$ is differentiable a.e. on $[a, b]$, and $f^{\prime}$ is measurable. If, in addition, $f$ is increasing on $[a, b]$ (note $f^{\prime} \geq 0$ where it exists), then $f^{\prime}$ is Lebesgue integrable over $[a, b]$, and

$$
\int_{a}^{b} f^{\prime}(x) d x \leq f(b)-f(a)
$$


where we write $\int_{a}^{b} f^{\prime}(x) d x$ instead of $\int_{[a, b]} f^{\prime}(x) d x$. Proof in [3] and [16]. It involves Proposition 2.39 (Fatou's Lemma) and ii of Proposition 2.34.

Definition 3.3: Let $f$ be a real-valued function defined on an interval $[a, b]$. Given a partition $P=\left\{x_{0}, x_{1}, \ldots, x_{n}\right\}$ of $[a, b]$, set $\Delta f_{i}=f\left(x_{i}\right)-f\left(x_{i-1}\right)$, $i=1, \ldots, n$, and define

$$
V(f ; a, b)=\sup _{P} \sum_{i=1}^{n}\left|\Delta f_{i}\right|,
$$

the supremum taken over all partitions $P$ of $[a, b]$. $f$ is said to be of bounded variation on $[a, b]$ if $V(f ; a, b)<\infty$.

Proposition 3.5 (Jordan decomposition): A function $f$ is of bounded variation on $[a, b]$ if and only if it is the difference of two monotonically increasing real-valued functions on $[a, b]$. Proof in [16] and [18].

Corollary 3.3: If $f$ is of bounded variation on $[a, b]$ then $f$ is differentiable a.e. on $[a, b]$, and $f^{\prime}$ is measurable and Lebesgue integrable over $[a, b]$.

Proof: By Proposition 3.5, $f=f_{1}-f_{2}$ on $[a, b]$, where $f_{1}$ and $f_{2}$ are monotonically increasing on $[a, b]$. Thus, by Proposition $3.4, f^{\prime}$ is measurable and exists a.e. on $[a, b]$. Since $\left|f^{\prime}\right| \leq\left|f_{1}^{\prime}\right|+\left|f_{2}^{\prime}\right|=f_{1}^{\prime}+f_{2}^{\prime}$ a.e. on $[a, b]$, then again by Proposition 3.4,

$$
\int_{a}^{b}\left|f^{\prime}(x)\right| d x \leq \int_{a}^{b} f_{1}^{\prime}(x) d x+\int_{a}^{b} f_{2}^{\prime}(x) d x \leq f_{1}(b)-f_{1}(a)+f_{2}(b)-f_{2}(a),
$$

and therefore $f^{\prime}$ is Lesbegue integrable over $[a, b]$ (Proposition 2.31).

Definition 3.4: Given a Lebesgue integrable function $f$ over $[a, b]$, and a real-valued function $F$ on $[a, b]$ such that

$$
F(x)=F(a)+\int_{a}^{x} f(t) d t, \quad x \in[a, b],
$$

then the function $F$ is said to be an indefinite integral of $f$ over $[a, b]$.

Proposition 3.6 (Indefinite integral of $f$ zero everywhere, then $f$ is 
zero a.e.): If $f$ is Lebesgue integrable over $[a, b]$ and $\int_{a}^{x} f(t) d t=0$ for all $x \in[a, b]$, then $f=0$ a.e. on $[a, b]$. Proof in [16]. It involves Proposition 2.15.

Proposition 3.7 (Differentiability of the indefinite integral): Let $f$ be Lebesgue integrable over an interval $[a, b]$, and $F$ a function such that

$$
F(x)=F(a)+\int_{a}^{x} f(t) d t, \quad x \in[a, b]
$$

i.e., an indefinite integral. Then $F^{\prime}=f$ a.e. on $[a, b]$.

Proof in [16]. It involves Proposition 3.6, Corollary 2.6 (Bounded Convergence Theorem), the inequality in Proposition 3.4, and i of Proposition 2.34.

Definition 3.5: A real-valued function $f$ defined on an interval $[a, b]$ is said to be absolutely continuous on $[a, b]$ if for every $\epsilon>0$ there is $\delta>0$ such that

$$
\sum_{i=1}^{n}\left|f\left(x_{i}^{\prime}\right)-f\left(x_{i}\right)\right|<\epsilon
$$

for any integer $n>0$ and any disjoint collection of open intervals $\left(x_{i}, x_{i}^{\prime}\right) \subseteq$ $[a, b], i=1, \ldots, n$, with

$$
\sum_{i=1}^{n}\left(x_{i}^{\prime}-x_{i}\right)<\delta .
$$

Proposition 3.8 (Absolutely continuous $f$ is constant if $f^{\prime}$ is zero a.e.): If $f$ is absolutely continuous on $[a, b]$ with $f^{\prime}=0$ a.e. on $[a, b]$, then $f$ is constant on $[a, b]$, i.e., $f(x)=f(a)$ for all $x \in[a, b]$. Proof in [16].

Observation 3.3: Absolutely continuous $\Rightarrow$ uniformly continuous $[18] \Rightarrow$ continuous. Moreover, a continuous real-valued function of compact domain is uniformly continuous [18]. Accordingly, a function $f$ called the Cantor function from $[0,1]$ onto $[0,1]$ that is continuous, thus uniformly continuous, but not absolutely continuous is described below. This function $f$ is monotonically increasing on $[0,1]$ and thus differentiable a.e. on $[0,1]$. Actually, $f^{\prime}=0$ at points not in the Cantor set (described in Observation 2.4) and does not exist at points in it. Thus, $f^{\prime}=0$ a.e. on $[0,1], f$ is not constant on $[0,1]$, hence $f$ can not be absolutely continuous on $[0,1]$ by Proposition 3.8.

For this purpose, we note that given $x \in[0,1], x$ can be expressed in its 
ternary expansion as $0 . a_{1} a_{2} a_{3} \cdots$ so that $x=\sum_{n=1}^{\infty} a_{n} / 3^{n}, a_{n} \in\{0,1,2\}$. Note $x=1$ is then expressed as $0.222 \cdots$. Similarly, given $y \in[0,1], y$ can be expressed in its binary expansion as $0 . b_{1} b_{2} b_{3} \cdots$ so that $y=\sum_{n=1}^{\infty} b_{n} / 2^{n}$, $b_{n} \in\{0,1\}$. Note $y=1$ is then expressed as $0.111 \cdots$.

In Observation 2.4 the Cantor set was identified as $\cap_{n=1}^{\infty} E_{n}$, where $E_{1}$ is the union of $[0,1 / 3]$ and $[2 / 3,1]$ obtained by removing the open middle third of $[0,1], E_{2}$ is the union of $[0,1 / 9],[2 / 9,3 / 9],[6 / 9,7 / 9],[8 / 9,1]$ obtained by removing the open middle thirds of $[0,1 / 3]$ and $[2 / 3,1]$, and so on. Actually, with $E_{0}=[0,1]$, then at stage $m$, open intervals of the form $\left((3 k-2) / 3^{m},(3 k-1) / 3^{m}\right), k \in\left\{1, \ldots, 3^{m-1}\right\}$, are removed from $E_{m-1}$, if contained in it, to obtain $E_{m}$. We note that endpoints of any such intervals have two ternary expansions, and in what follows, only the expansion of any such point that contains no 1's is considered. Fixing one of these removed open intervals, we note it is the open middle third of a closed interval in $E_{m-1}$, all numbers in the closed interval in $E_{m-1}$ having the same first $m-1$ digits in their ternary expansions, none of them equal to 1. Finally, we note numbers in the removed open interval have 1 as the $m^{\text {th }}$ digit of their ternary expansions, while numbers in the closed left and right thirds of the closed interval, closed thirds that become part of $E_{m}$, have 0 and 2, respectively, as the $m^{\text {th }}$ digit of their ternary expansions. Thus, the Cantor set is exactly the set of numbers in $[0,1]$ that have no 1 's in their ternary expansions.

An attempt can be made to identify the Cantor function as follows. Recalling that $(1 / 3,2 / 3)$ was the open middle third that was removed from $[0,1]$ to obtain $E_{1}$, given $x$ in its closure, i.e., in $[1 / 3,2 / 3]$, set $f(x)=1 / 2$. Again, recalling that $(1 / 9,2 / 9)$ and $(7 / 9,8 / 9)$ were the open middle thirds that were removed from $[0,1 / 3]$ and $[2 / 3,1]$, respectively, to obtain $E_{2}$, given $x$ in the closure of $(1 / 9,2 / 9)$, i.e., in $[1 / 9,2 / 9]$, set $f(x)=1 / 4$, and given $x$ in the closure of $(7 / 9,8 / 9)$, i.e., in $[7 / 9,8 / 9]$, set $f(x)=3 / 4$. Accordingly, $f$ can be identified this way at each stage of the contruction of the Cantor set but this is not enough as it has not been identified for points in "the limit" that are part of the Cantor set.

The Cantor function is properly identified as follows. Given $x \in[0,1]$ with ternary expansion $0 . a_{1} a_{2} a_{3} \cdots$ so that $x=\sum_{n=1}^{\infty} a_{n} / 3^{n}, a_{n} \in\{0,1,2\}$, let $N$ be the smallest $n$ such that $a_{n}$ equals 1 . If such an $n$ does not exist, i.e., $x$ is 
in the Cantor set, let $N=\infty$. With $b_{n}=a_{n} / 2$ if $n<N, b_{n}=1$ if $n=N$, and $b_{n}=0$ if $n>N$, let $y$ be the number in $[0,1]$ with binary expansion $0 . b_{1} b_{2} b_{3} \cdots$ so that $y=\sum_{n=1}^{\infty} b_{n} / 2^{n}=\sum_{n=1}^{N} b_{n} / 2^{n}$, and set $f(x)=y$. The function $f$ identified this way is then called the Cantor function.

Proposition 3.9: Let $f$ be the Cantor function. Then $f$ is continuous, thus uniformly continuous, from $[0,1]$ onto $[0,1]$. In addition, $f$ is monotonically increasing on $[0,1]$ and thus differentiable a.e. on $[0,1]$. Actually, $f^{\prime}=0$ at points not in the Cantor set and does not exist at points in it.

Proof: Given $x_{1}, x_{2} \in[0,1], x_{1}<x_{2}$, we show $f\left(x_{1}\right) \leq f\left(x_{2}\right)$.

Let $0 . a_{1} a_{2} a_{3} \cdots, 0 . c_{1} c_{2} c_{3} \cdots$ be $x_{1}, x_{2}$, respectively, in their ternary expansions. Let $0 . b_{1} b_{2} b_{3} \cdots, 0 . d_{1} d_{2} d_{3} \cdots$ be $f\left(x_{1}\right), f\left(x_{2}\right)$, respectively, in their binary expansions.

Let $N_{1}$ be the smallest $n$ such that $a_{n}=1 ; N_{1}=\infty$ if there is no such $n$.

Let $N_{2}$ be the smallest $n$ such that $c_{n}=1 ; N_{2}=\infty$ if there is no such $n$.

Let $N^{\prime}$ be the smallest $n$ such that $a_{n}<c_{n}$.

If $N^{\prime}>N_{1}$, since then $c_{n}=a_{n}, n=1, \ldots, N_{1}$, and, in particular, $c_{N_{1}}=$ $a_{N_{1}}=1$, it must be that $N_{2}=N_{1}$ so that $b_{n}=d_{n}, n=1, \ldots, N_{1}=N_{2}$, and therefore $f\left(x_{1}\right)=\sum_{n=1}^{N_{1}} b_{n} / 2^{n}=\sum_{n=1}^{N_{2}} d_{n} / 2^{n}=f\left(x_{2}\right)$.

Similarly if $N^{\prime}>N_{2}$, and the case $N^{\prime}=N_{1}=N_{2}$ can not be.

If $N^{\prime}=N_{1}$ and $N_{2}>N^{\prime}$, since $a_{N_{1}}=1$, it must be that $c_{N_{1}}=2$ so that $b_{n}=$ $d_{n}, n=1, \ldots, N_{1}-1, b_{N_{1}}=d_{N_{1}}=1$. Therefore, $\sum_{n=1}^{N_{1}} b_{n} / 2^{n}=\sum_{n=1}^{N_{1}} d_{n} / 2^{n}$ thus $f\left(x_{1}\right)=\sum_{n=1}^{N_{1}} b_{n} / 2^{n} \leq \sum_{n=1}^{N_{2}} d_{n} / 2^{n}=f\left(x_{2}\right)$.

If $N^{\prime}=N_{2}$ and $N_{1}>N^{\prime}$, since $c_{N_{2}}=1$, it must be that $a_{N_{2}}=0$ so that $b_{n}=d_{n}, n=1, \ldots, N_{2}-1, b_{N_{2}}=0, d_{N_{2}}=1$. Therefore, $\sum_{n=1}^{N_{2}} b_{n} / 2^{n}<$ $\sum_{n=1}^{N_{2}} d_{n} / 2^{n}$ thus $f\left(x_{1}\right)=\sum_{n=1}^{N_{1}} b_{n} / 2^{n} \leq \sum_{n=1}^{N_{2}} d_{n} / 2^{n}=f\left(x_{2}\right)$.

Finally, if $N_{1}>N^{\prime}, N_{2}>N^{\prime}$, since $a_{N^{\prime}}<c_{N^{\prime}}$, it must be that $a_{N^{\prime}}=0$, $c_{N^{\prime}}=2$, so that $b_{n}=d_{n}, n=1, \ldots, N^{\prime}-1, b_{N^{\prime}}=0, d_{N^{\prime}}=1$. Therefore, $\sum_{n=1}^{N^{\prime}} b_{n} / 2^{n}<\sum_{n=1}^{N^{\prime}} d_{n} / 2^{n}$ thus $f\left(x_{1}\right)=\sum_{n=1}^{N_{1}} b_{n} / 2^{n} \leq \sum_{n=1}^{N_{2}} d_{n} / 2^{n}=f\left(x_{2}\right)$. Thus, $f\left(x_{1}\right) \leq f\left(x_{2}\right)$ for all cases and therefore $f$ is monotonically increasing.

Given $y \in[0,1]$, we show there is $x \in[0,1]$ with $f(x)=y$.

Let $0 . b_{1} b_{2} b_{3} \cdots$ be $y$ in its binary expansion.

For each $n$, let $a_{n}=2 b_{n}$. Then for each $n, a_{n}$ is either zero or two.

Let $x$ be the point in $[0,1]$ which in its ternary expansion is $0 . a_{1} a_{2} a_{3} \cdots$.

Then $x$ is actually a point in the Cantor set and $f(x)=y$. 
Thus, $f$ is onto $[0,1]$.

That $f$ is continuous, thus uniformly continuous, on $[0,1]$, now follows from Corollary 3.2.

Finally, given $x \in[0,1]$, if $x$ is not in the Cantor set, we show $f^{\prime}(x)=0$. On the other hand, if $x$ is in the Cantor set, we show that $f^{\prime}(x)$ does not exist. If $x$ is not in the Cantor set, its ternary expansion must contain 1 as one of its digits. Then for some integer $m, m>0$, the $m^{\text {th }}$ digit of the expansion equals 1 with no previous digits equal to 1 . It follows that $x$ must be contained in an open interval of the form $\left((3 k-2) / 3^{m},(3 k-1) / 3^{m}\right)$, $k \in\left\{1, \ldots, 3^{m-1}\right\}$. Thus, it suffices to show $f$ is constant on any such interval. But this follows immediately since all numbers in the interval have the same first $m$ digits in their ternary expansions with 1 as the $m t h$ digit and no previous digits equal to 1 .

On the other hand, if $x$ is in the Cantor set, its ternary expansion consists of 0 's and 2's. Given an integer $n>0$, define $x_{n}$ to be the number in $[0,1]$ whose ternary expansion is exactly that of $x$ except at its $n^{\text {th }}$ digit. Its $n^{\text {th }}$ digit is 0 if the $n^{\text {th }}$ digit of $x$ is 2 , and it is 2 if that of $x$ is 0 . It follows then that $\left|x_{n}-x\right|=2 / 3^{n}$ so that $x_{n} \rightarrow x$. Also, $\left|f\left(x_{n}\right)-f(x)\right|=1 / 2^{n}$. Thus, since $f$ is monotonically increasing, $\left(f\left(x_{n}\right)-f(x)\right) /\left(x_{n}-x\right)=\left|f\left(x_{n}\right)-f(x)\right| /\left|x_{n}-x\right|=$ $(1 / 2)(3 / 2)^{n} \rightarrow \infty$ so that $f^{\prime}(x)$ does not exist.

Thus, $f^{\prime}$ does not exist at points in the Cantor set and equals zero otherwise.

Corollary 3.4: The Cantor function is not absolutely continuous on $[0,1]$.

Proof: Let $f$ be the Cantor function and assume it is absolutely continuous on $[0,1]$. By Proposition $3.9, f^{\prime}=0$ a.e. on $[0,1]$. Thus, by Proposition 3.8, $f$ must be constant on $[0,1]$, i.e., $f(x)=f(0)$ for all $x \in[0,1]$. But this is a contradiction as for instance $f(0)=0$ and $f(1)=1$. Thus, $f$ is not absolutely continuous on $[0,1]$.

Observation 3.4: For the sake of completeness, we analyze the nondifferentiability of the Cantor function $f$ on the Cantor set.

Let $x_{L}$ and $x_{R}$ be points in the Cantor set that are the left and right endpoints of an open interval $I$ removed at the $m^{\text {th }}$ stage of the construction of the Cantor set. It must then be that in their ternary expansions, $x_{L}$ 
can be expressed as $0 . a_{1} a_{2} \cdots a_{m-1} 0 \overline{2}$ (a bar on a digit means the digit is infinitely repeated), and $x_{R}$ as $0 . a_{1} a_{2} \cdots a_{m-1} 2 \overline{0}$, the set $\left\{a_{1}, a_{2}, \ldots, a_{m-1}\right\}$ with elements equal to 0 or 2 if $m>1$, empty if $m=1$. Given $x$ in the open interval $I$, it must be that in its ternary expansion the $m^{\text {th }}$ digit is 1 , and if $m>1$, then the first $m-1$ digits are also $a_{1}, a_{2}, \ldots, a_{m-1}$. Define for each integer $n, n>0$, a number $x_{L}^{n}$ in $I$ that in its ternary expansion the first $m$ digits are as described above, and all other digits are 0 except the $(m+n)^{t h}$ digit which is 1 . Then $\lim _{n \rightarrow \infty} x_{L}^{n}=x_{L}$ and $f\left(x_{L}^{n}\right)=f\left(x_{L}\right)$ for all $n$ so that $\lim _{n \rightarrow \infty}\left(f\left(x_{L}^{n}\right)-f\left(x_{L}\right)\right) /\left(x_{L}^{n}-x_{L}\right)=0$. Since for any sequence $\left\{x_{n}\right\}$ in $I$, with $\lim _{n \rightarrow \infty} x_{n}=x_{L}$, then $f\left(x_{n}\right)=f\left(x_{L}\right)$ for all $n$, it follows that $\left(f(t)-f\left(x_{L}\right)\right) /\left(t-x_{L}\right)$ has a limit as $t \rightarrow x_{L}$ from the right side of $x_{L}$ and it is zero. Similarly, define for each integer $n, n>0$, a number $x_{R}^{n}$ in $I$ that in its ternary expansion the first $m$ digits are as described above, and all other digits are 0 except the $(m+1)^{t h}, \ldots,(m+n)^{t h}$ digits which are 2. Then $\lim _{n \rightarrow \infty} x_{R}^{n}=x_{R}$ and $f\left(x_{R}^{n}\right)=f\left(x_{R}\right)$ for all $n$ so that $\lim _{n \rightarrow \infty}\left(f\left(x_{R}^{n}\right)-f\left(x_{R}\right)\right) /\left(x_{R}^{n}-x_{R}\right)=0$. Since for any sequence $\left\{x_{n}\right\}$ in $I$, with $\lim _{n \rightarrow \infty} x_{n}=x_{R}$, then $f\left(x_{n}\right)=f\left(x_{R}\right)$ for all $n$, it follows that $\left(f(t)-f\left(x_{R}\right)\right) /\left(t-x_{R}\right)$ has a limit as $t \rightarrow x_{R}$ from the left side of $x_{R}$ and it is zero.

In the proof of Proposition 3.9, given any $x$ in the Cantor set, a sequence $\left\{x_{n}\right\}$ of points in the Cantor set was identifed with $x_{n} \rightarrow x$ and $\left(f\left(x_{n}\right)-\right.$ $f(x)) /\left(x_{n}-x\right) \rightarrow \infty$. We show that with $x_{L}, x_{R}$ as above, then $(f(t)-$ $\left.f\left(x_{L}\right)\right) /\left(t-x_{L}\right)$ has a limit as $t \rightarrow x_{L}$ from the left side of $x_{L}$ and it is $\infty$, and $\left(f(t)-f\left(x_{R}\right)\right) /\left(t-x_{R}\right)$ has a limit as $t \rightarrow x_{R}$ from the right side of $x_{R}$ and it is also $\infty$. Actually, we only show it for $x_{R}$ as the proof for $x_{L}$ can be similarly accomplished. Accordingly, let $n \geq m$ be an integer such that the ternary expansions of $t$ and $x_{R}$ coincide in the first $n$ digits and the $(n+1)^{t h}$ digit of $t$ is 1 or 2 . As mentioned above, all digits of $x_{R}$ after the $m^{\text {th }}$ digit equal 0 . Thus, $f(t)-f\left(x_{R}\right) \geq 1 / 2^{n+1}$ and $t-x_{R} \leq 2 / 3^{n+1}+2 / 3^{n+2}+\cdots=1 / 3^{n}$, so that

$$
\lim _{t \rightarrow x_{R}^{+}} \frac{f(t)-f\left(x_{R}\right)}{t-x_{R}} \geq \lim _{n \rightarrow \infty}(1 / 2)(3 / 2)^{n}=\infty .
$$

Finally, it is of interest to note that if $x$ is any point in the Cantor set, then at stage $m$ of the contruction of the Cantor set, $x$ is in a closed interval $\left[a_{m}, b_{m}\right] \subset[0,1]$, where if $0 . x_{1} x_{2} \cdots$ is $x$ in its ternary expansion, then $0 . x_{1}$. $\cdots x_{m} \overline{0}$ is $a_{m}$ in its ternary expansion, and $0 . x_{1} \cdots x_{m} \overline{2}$ is $b_{m}$ in its ternary expansion. It follows that $b_{m}-a_{m}=\sum_{i=m+1}^{\infty} 2 / 3^{i}=1 / 3^{m}$ and $f\left(b_{m}\right)-$ 
$f\left(a_{m}\right)=\sum_{i=m+1}^{\infty} 1 / 2^{i}=1 / 2^{m}$. Thus, with $a_{m} \leq x \leq b_{m}$, we have

$$
\lim _{m \rightarrow \infty} \frac{f\left(b_{m}\right)-f\left(a_{m}\right)}{b_{m}-a_{m}}=\lim _{m \rightarrow \infty}(3 / 2)^{m}=\infty .
$$

If $x=x_{L}, x_{L}$ as above, then for some $m, x=b_{m}$ and $\lim _{m \rightarrow \infty}(f(x)-$ $\left.f\left(a_{m}\right)\right) /\left(x-a_{m}\right)=\infty$, as expected. Similarly, if $x=x_{R}, x_{R}$ as above, then for some $m, x=a_{m}$ and $\lim _{m \rightarrow \infty}\left(f(x)-f\left(b_{m}\right)\right) /\left(x-b_{m}\right)=\infty$, also as expected. As for a point $x$ in the Cantor set that is not an endpoint of an open interval removed at some stage of the construction of the Cantor set, it is easier to see that $\lim _{m \rightarrow \infty}\left(f(x)-f\left(a_{m}\right)\right) /\left(x-a_{m}\right)=\infty$, and $\lim _{m \rightarrow \infty}(f(x)-$ $\left.f\left(b_{m}\right)\right) /\left(x-b_{m}\right)=\infty$, by looking at the ternary expansions of $x, a_{m}$ and $b_{m}$. Actually, we only show it for $\left\{a_{m}\right\}$ as the proof for $\left\{b_{m}\right\}$ can be similarly accomplished. Accordingly, let $m>0$ be an integer such that the $(m+1)^{t h}$ digit of $x$ in its ternary expansion, i.e., $x_{m+1}$, equals 2 . As mentioned above, the ternary expansions of $x$ and $a_{m}$ coincide in the first $m$ digits and all digits of $a_{m}$ after the $m^{\text {th }}$ digit equal 0 . Thus, $f(x)-f\left(a_{m}\right) \geq 1 / 2^{m+1}$ and $x-a_{m} \leq 2 / 3^{m+1}+2 / 3^{m+2}+\cdots=1 / 3^{m}$, so that

$$
\lim _{m \rightarrow \infty} \frac{f(x)-f\left(a_{m}\right)}{x-a_{m}} \geq \lim _{n \rightarrow \infty}(1 / 2)(3 / 2)^{m}=\infty .
$$

Note that $\lim _{m \rightarrow \infty}\left(f(x)-f\left(a_{m}\right)\right) /\left(x-a_{m}\right)=\lim _{m \rightarrow \infty}\left(f(x)-f\left(b_{m}\right)\right) /(x-$ $\left.b_{m}\right)=\infty$ does not imply that $\lim _{t \rightarrow x}(f(x)-f(t)) /(x-t)=\infty$.

Observation 3.5: A function on $[a, b]$ that is a finite linear combination of absolutely continuous functions on $[a, b]$ is absolutely continuous on $[a, b]$. The proof is analogous to the proof that a finite linear combination of continuous functions is continuous. In addition, the product of two absolutely continuous functions on $[a, b]$ is absolutely continuous on $[a, b]$.

Proposition 3.10 (Absolutely continuous $f$ implies $f$ is of bounded variation): If $f$ is absolutely continuous on $[a, b]$, then $f$ is of bounded variation on $[a, b]$. Proof in [16].

Corollary 3.5: If $f$ is absolutely continuous on $[a, b]$ then $f$ is differentiable a.e. on $[a, b]$, and $f^{\prime}$ is measurable and Lebesgue integrable over $[a, b]$. 
Proposition 3.11 (Absolute continuity of the indefinite integral): If $F$ is an indefinite integral over $[a, b]$, then $F$ is absolutely continuous on $[a, b]$.

Proof: Assume (Definition 3.4) $F(x)=F(a)+\int_{a}^{x} f(t) d t, x \in[a, b], f$ is Lebesgue integrable over $[a, b]$. By Proposition 2.31, $|f|$ is Lebesgue integrable over $[a, b]$. Then by Proposition 2.38, given $\epsilon>0$ there is $\delta>0$ such that for each measurable set $A \subseteq[a, b]$ with $m(A)<\delta$, then $\int_{A}|f(t)| d t<\epsilon$. Given integer $n>0$ and disjoint open intervals $\left(x_{i}, x_{i}^{\prime}\right) \subseteq[a, b], i=1, \ldots, n$, with $\sum_{i=1}^{n}\left(x_{i}^{\prime}-x_{i}\right)<\delta$, let $A=\cup_{i=1}^{n}\left(x_{i}, x_{i}^{\prime}\right)$. Then $A$ is measurable and $m(A)<\delta$. Thus, $\int_{A}|f(t)| d t<\epsilon$. Accordingly, then

$$
\begin{aligned}
\sum_{i=1}^{n}\left|F\left(x_{i}^{\prime}\right)-F\left(x_{i}\right)\right| & =\sum_{i=1}^{n}\left|\int_{a}^{x_{i}^{\prime}} f(t) d t-\int_{a}^{x_{i}} f(t) d t\right|=\sum_{i=1}^{n}\left|\int_{x_{i}}^{x_{i}^{\prime}} f(t) d t\right| \\
& \leq \sum_{i=1}^{n} \int_{x_{i}}^{x_{i}^{\prime}}|f(t)| d t=\int_{A}|f(t)| d t<\epsilon .
\end{aligned}
$$

Thus, $F$ is absolutely continuous on $[a, b]$.

Proposition 3.12 (Equivalent conditions for an absolutely continuous function): Given a real-valued function $f$ on $[a, b]$, then the following three conditions are equivalent:

i. $f$ is absolutely continuous on $[a, b]$.

ii. There exists a Lebesgue integrable function $g$ over $[a, b]$ such that $f(x)=f(a)+\int_{a}^{x} g(t) d t, x \in[a, b]$.

(Note that then by Proposition 3.7, $f^{\prime}=g$ a.e. on $[a, b]$ ).

iii. $f^{\prime}$ exists a.e. on $[a, b]$ and is Lebesgue integrable over $[a, b]$, and $f(x)=f(a)+\int_{a}^{x} f^{\prime}(t) d t, x \in[a, b]$.

\section{Proof:}

iii $\Rightarrow$ ii:

Take $g=f^{\prime}$.

ii $\Rightarrow$ i:

This is Proposition 3.11 .

$\mathrm{i} \Rightarrow$ iii:

By Corollary 3.5, $f^{\prime}$ exists a.e. on $[a, b]$ and is Lebesgue integrable over $[a, b]$. Let $G(x)=\int_{a}^{x} f^{\prime}(t) d t, x \in[a, b]$. Then $G$ is an indefinite integral of $f^{\prime}$ over $[a, b]$ and by Proposition 3.11, is absolutely continuous on $[a, b]$, and so 
is the function $h=f-G$ by Observation 3.5.

By Proposition 3.7, $G^{\prime}=f^{\prime}$ a.e. on $[a, b]$. Thus $h^{\prime}=0$ a.e. on $[a, b]$, and by Proposition 3.8, $h$ is constant on $[a, b]$, i.e., $f-G=C$ on $[a, b]$ for some constant $C$, i.e., $f(x)-\int_{a}^{x} f^{\prime}(t) d t=C, x \in[a, b]$.

Since $C=f(a)$, it then follows that $f(x)=f(a)+\int_{a}^{x} f^{\prime}(t) d t, x \in[a, b]$.

Corollary 3.6 (Fundamental Theorem of Lebesgue integral calculus): Given real-valued functions $f, g$ on $[a, b], f$ absolutely continuous on $[a, b]$ and $f^{\prime}=g$ a.e. on $[a, b]$, then $f(x)=f(a)+\int_{a}^{x} g(t) d t, x \in[a, b]$.

Proposition 3.13 (Fundamental Theorem of Lebesgue integral calculus (Alternate form)): Given a real-valued function $f$ on $[a, b]$, if $f^{\prime}(x)$ exists for every $x \in[a, b]$, and $f^{\prime}$ is Lebesgue integrable over $[a, b]$, then $f(x)=f(a)+\int_{a}^{x} f^{\prime}(t) d t, x \in[a, b]$. Proof in [19].

Corollary 3.7: Given a real-valued function $f$ on $[a, b]$, if $f^{\prime}$ exists everywhere on $[a, b]$ and $f^{\prime}$ is Lebesgue integrable over $[a, b]$, then $f$ is absolutely continuous on $[a, b]$.

Proof: From Proposition 3.13 and then Proposition 3.12.

Proposition 3.14 (Change of variable for Riemann integral): Given a strictly monotonic continuous function $u$ from an interval $[a, b]$ onto an interval $[c, d](u(a)=c, u(b)=d$ if $u$ is strictly increasing, $u(a)=d, u(b)=c$ if it is strictly decreasing), with $u^{\prime}$ Riemann integrable over $[a, b]$, and a real-valued Riemann integrable function $f$ over $[c, d]$, then

$$
\mathcal{R} \int_{u(a)}^{u(b)} f(x) d x=\mathcal{R} \int_{a}^{b} f(u(t)) u^{\prime}(t) d t
$$

with $\mathcal{R} \int_{u(a)}^{u(b)} f(x) d x=-\mathcal{R} \int_{c}^{d} f(x) d x$ if $u$ is decreasing. Proof in [1] and [18]. Note that by Proposition $3.4, u^{\prime}$ exists a.e. on $[a, b]$.

Proposition 3.15 (Substitution rule for Riemann integral): Given a function $u$ from an interval $[a, b]$ into an interval $I$ such that $u^{\prime}(x)$ exists for every $x \in[a, b]$ with $u^{\prime}$ Riemann integrable over $[a, b]$, and a real-valued 
continuous function $f$ on $I$, then

$$
\mathcal{R} \int_{u(a)}^{u(b)} f(x) d x=\mathcal{R} \int_{a}^{b} f(u(t)) u^{\prime}(t) d t
$$

with $\mathcal{R} \int_{u(a)}^{u(b)} f(x) d x=-\mathcal{R} \int_{u(b)}^{u(a)} f(x) d x$ if $u(b)<u(a)$.

Proof: By Proposition 3.2 (Fundamental Theorem of calculus II), with $e$ the left endpoint of $I$, the function $F$ defined by $F(x)=\mathcal{R} \int_{e}^{x} f(t) d t, x \in I$, satisfies $F^{\prime}(x)=f(x)$ for every $x \in I$ since $f$ is continuous on $I$. From the definition of $F$, given $c, d \in I, c$ not necessarily less than $d$, then $F(d)-$ $F(c)=\mathcal{R} \int_{c}^{d} f(x) d x$. In particular,

$$
F(u(b))-F(u(a))=\mathcal{R} \int_{u(a)}^{u(b)} f(x) d x .
$$

Since $u$ is differentiable on $[a, b]$ and $F$ is differentiable on $I$, the composite function $F \circ u$ is differentiable on $[a, b]$ and by the usual chain rule of calculus, $(F \circ u)^{\prime}(t)=F^{\prime}(u(t)) u^{\prime}(t)=f(u(t)) u^{\prime}(t)$, for every $t \in[a, b]$. Thus, since $f(u(t)) u^{\prime}(t)$ is clearly Riemann integrable over $[a, b]$, by Proposition 3.1 (Fundamental Theorem of calculus I), it must be that

$$
\begin{aligned}
\mathcal{R} \int_{a}^{b} f(u(t)) u^{\prime}(t) d t & =\mathcal{R} \int_{a}^{b}(F \circ u)^{\prime}(t) d t=(F \circ u)(b)-(F \circ u)(a) \\
& =F(u(b))-F(u(a))=\mathcal{R} \int_{u(a)}^{u(b)} f(x) d x
\end{aligned}
$$

Proposition 3.16 (Substitution rule for Lebesgue integral): Given an absolutely continuous function $u$ from an interval $[a, b]$ into an interval $I$, and a real-valued continuous function $f$ on $I$, then

$$
\int_{u(a)}^{u(b)} f(x) d x=\int_{a}^{b} f(u(t)) u^{\prime}(t) d t
$$

with $\int_{u(a)}^{u(b)} f(x) d x=-\int_{u(b)}^{u(a)} f(x) d x=-\int_{[u(b), u(a)]} f(x) d x$ if $u(b)<u(a)$.

Proof: By Proposition 3.2 (Fundamental Theorem of calculus II), with $e$ the left endpoint of $I$, the function $F$ defined by $F(x)=\mathcal{R} \int_{e}^{x} f(t) d t, x \in I$, satisfies $F^{\prime}(x)=f(x)$ for every $x \in I$ since $f$ is continuous on $I$. From the 
definition of $F$, given $c, d \in I, c$ not necessarily less than $d$, then $F(d)-$ $F(c)=\mathcal{R} \int_{c}^{d} f(x) d x$. In particular,

$$
F(u(b))-F(u(a))=\mathcal{R} \int_{u(a)}^{u(b)} f(x) d x=\int_{u(a)}^{u(b)} f(x) d x,
$$

where the last equation is by Proposition 2.33 (Riemann implies Lebesgue). Since $u$ is differentiable a.e. on $[a, b]$ and $F$ is differentiable on $I$, the composite function $F \circ u$ is differentiable a.e. on $[a, b]$. Indeed it is differentiable exactly at the points where $u$ is differentiable. Thus, by the usual chain rule of calculus, $(F \circ u)^{\prime}(t)=F^{\prime}(u(t)) u^{\prime}(t)=f(u(t)) u^{\prime}(t)$, for $t \in[a, b]$ at which $u^{\prime}$ exists.

Finally, we show $F \circ u$ is absolutely continuous on $[a, b]$ in order to use Corollary 3.6 (Fundamental Theorem of Lebesgue integral calculus) with $F \circ u$ as the absolutely continuous function in the hypothesis of the corollary. For this purpose, since $f$ is continuous, assume $|f|<M$ on $[a, b]$, for some $M>0$. Given $\epsilon>0$, let $\delta>0$ correspond to $\epsilon / M$ in the definition of the absolute continuity of $u$. Given integer $n>0$ and disjoint open intervals $\left(t_{i}, t_{i}^{\prime}\right) \subseteq[a, b]$, $i=1, \ldots, n$, with $\sum_{i=1}^{n}\left(t_{i}^{\prime}-t_{i}\right)<\delta$, then

$$
\begin{aligned}
\sum_{i=1}^{n}\left|F \circ u\left(t_{i}^{\prime}\right)-F \circ u\left(t_{i}\right)\right| & =\sum_{i=1}^{n}\left|\mathcal{R} \int_{u\left(t_{i}\right)}^{u\left(t_{i}^{\prime}\right)} f(x) d x\right| \\
& <\sum_{i=1}^{n}\left|u\left(t_{i}\right)-u\left(t_{i}^{\prime}\right)\right| M=M \sum_{i=1}^{n}\left|u\left(t_{i}\right)-u\left(t_{i}^{\prime}\right)\right| \\
& <M \epsilon / M=\epsilon .
\end{aligned}
$$

Thus, $F \circ u$ is absolutely continuous and by Corollary 3.6, it must be that

$$
\begin{aligned}
\int_{a}^{b} f(u(t)) u^{\prime}(t) d t & =\int_{a}^{b}(F \circ u)^{\prime}(t) d t=(F \circ u)(b)-(F \circ u)(a) \\
& =F(u(b))-F(u(a))=\int_{u(a)}^{u(b)} f(x) d x
\end{aligned}
$$

Observation 3.6: Note that in the proof of Proposition 3.16 above, while proving that $F \circ u$ is absolutely continuous on $[a, b]$, we have actually proved that if $u,[a, b], I, e$ are as given there and $f$ is Lebesgue integrable over $I$ and bounded on $I$, and the function $F$ is defined by $F(x)=\int_{e}^{x} f(t) d t, x \in I$, then $F \circ u$ is absolutely continuous on $[a, b]$. At the end of this section, results are presented for carrying out a change of variable in Lebesgue integrals, useful 
in shape analysis.

Proposition 3.17 (Saks' inequality [20]): Given a real-valued function $f$ on $[a, b]$, a real number $r \geq 0$, and $E \subseteq[a, b]$ such that $\left|f^{\prime}(x)\right| \leq r$ for each $x \in E$, then

$$
m^{*}(f(E)) \leq r m^{*}(E)
$$

Proof: Given $\epsilon>0$, for every integer $n>0$, and any $y \in(a, b)$, define

$$
E_{n}=\{x \in E: \text { if } 0<|x-y|<1 / n \text {, then }|f(x)-f(y)|<(r+\epsilon)|x-y|\} .
$$

Since $E_{n} \subseteq E_{n+1}$ for all $n$ and $\cup_{n=1}^{\infty} E_{n}=E$, then by 2 of Proposition 2.13, $m^{*}(E)=\lim _{n \rightarrow \infty} m^{*}\left(E_{n}\right)$. Similarly, since $f\left(E_{n}\right) \subseteq f\left(E_{n+1}\right)$ for all $n$ and $\cup_{n=1}^{\infty} f\left(E_{n}\right)=f(E)$, then again by 2 of Proposition 2.13,

$$
m^{*}(f(E))=\lim _{n \rightarrow \infty} m^{*}\left(f\left(E_{n}\right)\right) .
$$

Given integer $n>0$, let $\left\{I_{k}\right\}$ be a countable collection of open intervals covering $E_{n}$, i.e., $E_{n} \subseteq \cup_{k=1}^{\infty} I_{k}$, with

$$
\sum_{k=1}^{\infty} m\left(I_{k}\right)<m^{*}\left(E_{n}\right)+\epsilon
$$

Note $\left\{I_{k}\right\}$ can be chosen so that $m\left(I_{k}\right)<1 / n$ for each $k$. Then, for each $k$, given $x, x^{\prime} \in E_{n} \cap I_{k}, x \neq x^{\prime}$, from the definition of $E_{n}$, since $\left|x-x^{\prime}\right|<1 / n$, it must be that

$$
\left|f(x)-f\left(x^{\prime}\right)\right|<(r+\epsilon)\left|x-x^{\prime}\right|<(r+\epsilon) m\left(I_{k}\right) .
$$

Thus,

$$
m^{*}\left(f\left(E_{n} \cap I_{k}\right)\right) \leq \sup _{x, x^{\prime} \in E_{n} \cap I_{k}}\left|f(x)-f\left(x^{\prime}\right)\right| \leq(r+\epsilon) m\left(I_{k}\right) .
$$

Since $E_{n}=\cup_{k=1}^{\infty}\left(E_{n} \cap I_{k}\right)$, then $f\left(E_{n}\right)=\cup_{k=1}^{\infty} f\left(E_{n} \cap I_{k}\right)$, therefore,

$$
\begin{aligned}
m^{*}\left(f\left(E_{n}\right)\right) & \leq \sum_{k=1}^{\infty} m^{*}\left(f\left(E_{n} \cap I_{k}\right)\right) \leq \sum_{k=1}^{\infty}(r+\epsilon) m\left(I_{k}\right) \\
& =(r+\epsilon) \sum_{k=1}^{\infty} m\left(I_{k}\right)<(r+\epsilon)\left(m^{*}\left(E_{n}\right)+\epsilon\right) .
\end{aligned}
$$


Thus, since as established above $m^{*}(E)=\lim _{n \rightarrow \infty} m^{*}\left(E_{n}\right)$ and $m^{*}(f(E))=$ $\lim _{n \rightarrow \infty} m^{*}\left(f\left(E_{n}\right)\right)$, it must be that

$$
\begin{aligned}
m^{*}(f(E)) & =\lim _{n \rightarrow \infty} m^{*}\left(f\left(E_{n}\right)\right) \\
& \leq \lim _{n \rightarrow \infty}(r+\epsilon)\left(m^{*}\left(E_{n}\right)+\epsilon\right) \\
& =(r+\epsilon)\left(m^{*}(E)+\epsilon\right) .
\end{aligned}
$$

Hence, since $\epsilon>0$ is arbitrary, it must be that $m^{*}(f(E)) \leq r m^{*}(E)$.

Corollary 3.8: Given a real-valued function $f$ on $[a, b]$, let $E$ be a subset of $[a, b]$ on which $f^{\prime}=0$. Then $m(f(E))=m^{*}(f(E))=0$.

Proof: By Proposition 3.17, $m^{*}(f(E)) \leq 0 \cdot m^{*}(E)=0$. Thus, $m(f(E))=0$.

Corollary 3.9: Given a real-valued function $f$ on $[a, b]$, and $E \subseteq[a, b]$ with $m(E)=0$, such that $f^{\prime}$ exists on $E$, then $m(f(E))=m^{*}(f(E))=0$.

Proof: Without any loss of generality assume $E \subseteq(a, b)$. Given $\epsilon>0$, let $\left\{I_{k}\right\}$ be a countable collection of open intervals covering $E$, i.e., $E \subseteq \cup_{k=1}^{\infty} I_{k}$, with $\sum_{k=1}^{\infty} m\left(I_{k}\right)<\epsilon, I_{k} \subseteq(a, b)$ for each $k$.

Given an integer $l \geq 0$, and an integer $k>0$, let

$$
E_{l}=\left\{x \in E: f^{\prime}(x) \text { exists and }\left|f^{\prime}(x)\right| \leq l\right\}
$$

and

$$
E_{l k}=\left\{x \in I_{k}: f^{\prime}(x) \text { exists and }\left|f^{\prime}(x)\right| \leq l\right\} .
$$

Note $E_{l} \subseteq \cup_{k=1}^{\infty} E_{l k}$ so that $f\left(E_{l}\right) \subseteq \cup_{k=1}^{\infty} f\left(E_{l k}\right)$.

By Proposition 3.17, $m^{*}\left(f\left(E_{l k}\right)\right) \leq l m^{*}\left(E_{l k}\right)$ for each $k$, and therefore,

$$
m^{*}\left(f\left(E_{l}\right)\right) \leq \sum_{k=1}^{\infty} m^{*}\left(f\left(E_{l k}\right)\right) \leq \sum_{k=1}^{\infty} l m^{*}\left(E_{l k}\right) \leq \sum_{k=1}^{\infty} l m\left(I_{k}\right)<l \epsilon .
$$

Since $\epsilon>0$ is arbitrary, then $m^{*}\left(f\left(E_{l}\right)\right)=0$.

Finally, note $f\left(E_{l+1}\right) \supseteq f\left(E_{l}\right)$ for each $l$, and $f(E)=\cup_{l=0}^{\infty} f\left(E_{l}\right)$.

Thus, by 2 of Proposition 2.13, $m^{*}(f(E))=\lim _{l \rightarrow \infty} m^{*}\left(f\left(E_{l}\right)\right)=0$.

Corollary 3.10 (Saks' Theorem [20]): Given a real-valued function $f$ 
on $[a, b]$, and $E \subseteq[a, b]$ such that $f^{\prime}$ exists on $E$, if $f^{\prime}=0$ a.e. on $E$, then $m(f(E))=0$.

Proof: Let $E_{1}, E_{2}$ be subsets of $E, E=E_{1} \cup E_{2}, f^{\prime}=0$ on $E_{1}$ and $m\left(E_{2}\right)=0$. By Corollary 3.8, $m\left(f\left(E_{1}\right)\right)=0$. By Corollary 3.9, $m\left(f\left(E_{2}\right)\right)=0$. Thus, $m(f(E)) \leq m\left(f\left(E_{1}\right)\right)+m\left(f\left(E_{2}\right)\right)=0$.

Observation 3.7: Let $f$ be the Cantor function and $C$ the Cantor set. Then $f^{\prime}=0$ a.e. on $[0,1]$ and $m(C)=0$. Since $f([0,1])=[0,1]$, by Saks' Theorem (Corollary 3.10), it must be that $f$ is not differentiable at certain points in $[0,1]$, and since $f(C)=[0,1]$, by Corollary 3.9, it must be that $f$ is not differentiable at certain points in $C$. Of course we know $f$ is not differentiable at any point in $C$ and $f^{\prime}=0$ on $[0,1] \backslash C$ so that by Corollary 3.8, $m(f([0,1] \backslash$ $C))=0$ which makes sense as $f([0,1] \backslash C)$ is countable.

The following proposition is the converse of Saks' Theorem (Corollary 3.10): $m(f(E))=0$ implies $f^{\prime}=0$ a.e. on $E$. Here again it is assumed $f^{\prime}$ exists everywhere on $E$. However, almost everywhere (a.e.) will suffice.

Proposition 3.18 (Serrin-Varberg's Theorem [22]): Given $f$, a realvalued function on $[a, b]$, and $E \subseteq[a, b]$ such that $f^{\prime}$ exists on $E$, if $m(f(E))=$ 0 , then $f^{\prime}=0$ a.e. on $E$.

Proof: Let $B=\left\{x \in E:\left|f^{\prime}(x)\right|>0\right\}$, and for every integer $n>0$, and any $y \in(a, b)$, define

$$
B_{n}=\{x \in B \text { : if } 0<|x-y|<1 / n \text {, then }|f(x)-f(y)| \geq|x-y| / n\} .
$$

Clearly, we need to show $m(B)=0$, and since $B=\cup_{n=1}^{\infty} B_{n}$, then it suffices to show $m\left(B_{n}\right)=0$ for each $n$. However, since each $B_{n}$ can be covered by a countable collection of intervals, each interval of length less than $1 / n$, it then suffices to show that if $I$ is any interval of length less than $1 / n$ and $A=I \cap B_{n}$, then $m(A)=0$.

For this purpose, given $\epsilon>0$, since $A \subseteq E$ so that $m(f(A))=0$, let $\left\{I_{k}\right\}$ be a countable collection of open intervals covering $f(A)$, i.e., $f(A) \subseteq \cup_{k=1}^{\infty} I_{k}$, with $\sum_{k=1}^{\infty} m\left(I_{k}\right)<\epsilon$. In addition, let $A_{k}=f^{-1}\left(f(A) \cap I_{k}\right)$. Then $A=$ $\cup_{k=1}^{\infty} A_{k}$, and since for each $k, A_{k} \subseteq A=I \cap B_{n}$, given $x, x^{\prime} \in A_{k}, x \neq x^{\prime}$, then $\left|x-x^{\prime}\right|<1 / n$, and it must be that

$$
\left|x-x^{\prime}\right| \leq n\left|f(x)-f\left(x^{\prime}\right)\right|<n m\left(I_{k}\right) .
$$


Thus,

$$
m^{*}\left(A_{k}\right) \leq \sup _{x, x^{\prime} \in A_{k}}\left|x-x^{\prime}\right| \leq n m\left(I_{k}\right)
$$

and then

$$
m^{*}(A) \leq \sum_{k=1}^{\infty} m^{*}\left(A_{k}\right) \leq \sum_{k=1}^{\infty} n m\left(I_{k}\right)=n \sum_{k=1}^{\infty} m\left(I_{k}\right)<n \epsilon .
$$

Hence, since $\epsilon>0$ is arbitrary, it must be that $m(A)=0$.

Corollary 3.11: Given a real-valued function $f$ on $[a, b]$, and $E \subseteq[a, b]$ such that $f^{\prime}$ exists on $E$, if $f$ is constant on $E$, then $f^{\prime}=0$ a.e. on $E$.

Corollary 3.12 (Serrin-Varberg's Theorem (Alternate form)): Given a real-valued function $f$ on $[a, b]$, and $E \subseteq[a, b]$ such that $f^{\prime}$ exists a.e. on $E$, if $m(f(E))=0$, then $f^{\prime}=0$ a.e. on $E$. In particular, if $f$ is of bounded variation on $[a, b]$, and $E \subseteq[a, b]$ with $m(f(E))=0$, then $f^{\prime}=0$ a.e. on $E$.

Proof: Let $E_{1}, E_{2}$ be subsets of $E, E=E_{1} \cup E_{2}, f^{\prime}$ exists on $E_{1}$ and $m\left(E_{2}\right)=$ 0 . Since $m\left(f\left(E_{1}\right)\right) \leq m(f(E))=0$, then by Proposition 3.18, $f^{\prime}=0$ a.e. on $E_{1}$. Thus, since $m\left(E_{2}\right)=0$, then $f^{\prime}=0$ a.e. on $E$. If $f$ is of bounded variation on $[a, b]$, then by Corollary $3.3, f$ is differentiable a.e. on $[a, b]$ and therefore on any subset $E$ of $[a, b]$.

Proposition 3.19 (Measurability of the derivative of a measurable function): Let $f$ be a real-valued measurable function on $[a, b]$, and $E$ a measurable subset of $[a, b]$. If $f^{\prime}$ exists on $E$, then $f^{\prime}$ is a measurable function on E. Proof in [26].

Proposition 3.20: Let $f$ be a real-valued measurable function on $[a, b]$, and $E$ a measurable subset of $[a, b]$. If $f^{\prime}(x)$ exists for each $x \in E$, then

$$
m^{*}(f(E)) \leq \int_{E}\left|f^{\prime}(x)\right| d x .
$$

Proof: Given $\epsilon>0$, for each integer $n>0$, define

$$
E_{n}=\left\{x \in E:(n-1) \epsilon \leq\left|f^{\prime}(x)\right|<n \epsilon\right\}
$$


Clearly, the $E_{n}$ 's are pairwise disjoint and $E=\cup_{n=1}^{\infty} E_{n}$ so that $f(E)=$ $\cup_{n=1}^{\infty} f\left(E_{n}\right)$. By Proposition 3.19, $f^{\prime}$ is measurable on $E$. Hence, each set $E_{n}$ must be measurable. Since $f^{\prime}(x)$ exists for every $x \in E_{n}$, by Proposition 3.17, $m^{*}\left(f\left(E_{n}\right)\right) \leq n \epsilon m\left(E_{n}\right)$ for each $n$, and given an integer $N>0$, it must be that $\sum_{n=1}^{N}(n-1) \epsilon m\left(E_{n}\right) \leq \int_{E}\left|f^{\prime}(x)\right| d x$ by the definition of the Lebesgue integral, so that $\sum_{n=1}^{\infty}(n-1) \epsilon m\left(E_{n}\right) \leq \int_{E}\left|f^{\prime}(x)\right| d x$. Thus,

$$
\begin{aligned}
m^{*}(f(E)) & \leq \sum_{n=1}^{\infty} m^{*}\left(f\left(E_{n}\right)\right) \leq \sum_{n=1}^{\infty} n \epsilon m\left(E_{n}\right)=\sum_{n=1}^{\infty}((n-1) \epsilon+\epsilon) m\left(E_{n}\right) \\
& =\sum_{n=1}^{\infty}(n-1) \epsilon m\left(E_{n}\right)+\sum_{n=1}^{\infty} \epsilon m\left(E_{n}\right) \leq \int_{E}\left|f^{\prime}(x)\right| d x+\epsilon m(E)
\end{aligned}
$$

where, in the last step, the countable additivity of $m$ on measurable sets is used. Since $\epsilon$ is arbitrary and $m(E)<\infty$, then $m^{*}(f(E)) \leq \int_{E}\left|f^{\prime}(x)\right| d x$.

Proposition 3.21 (Absolutely continuous $f$ maps zero-measure sets to zero-measure sets [20] - Absolutely continuous $f$ maps measurable sets to measurable sets): Let $f$ be an absolutely continuous function on $[a, b]$. If $E \subseteq[a, b]$ with $m(E)=0$, then $m(f(E))=0$. In addition, given any measurable subset $E$ of $[a, b]$, then $f(E)$ is measurable.

Proof: Without any loss of generality assume $E \subseteq(a, b)$. Given $\epsilon>0$, let $\delta>0$ correspond to $\epsilon$ in the definition of the absolute continuity of $f$. Since $m(E)=0$, then by Proposition 2.1, there is a collection $\left\{I_{k}\right\}=$ $\left\{\left(a_{k}, b_{k}\right)\right\}$ of nonoverlapping open intervals covering $E$, i.e., $E \subseteq \cup_{k=1}^{\infty} I_{k}$, with $\sum_{k=1}^{\infty}\left(b_{k}-a_{k}\right)=\sum_{k=1}^{\infty} m\left(I_{k}\right)<\delta, I_{k} \subseteq(a, b)$ for each $k$.

For each $k$, since $f$ is continuous, let $c_{k}$ and $d_{k}$ be points in $\left[a_{k}, b_{k}\right]$ where $f$ attains its minimum and maximum, respectively. Assuming without any loss of generality that $c_{k}<d_{k}$, then $\left\{\left(c_{k}, d_{k}\right)\right\}$ is a collection of nonoverlapping open intervals, and since again $f$ is continuous, by the intermediate value theorem [18], it follows that

$$
f(E) \subseteq f\left(\cup_{k=1}^{\infty}\left(a_{k}, b_{k}\right)\right)=\cup_{k=1}^{\infty} f\left(\left(a_{k}, b_{k}\right)\right) \subseteq \cup_{k=1}^{\infty} f\left(\left[c_{k}, d_{k}\right]\right) .
$$

Thus,

$$
m^{*}(f(E)) \leq \sum_{k=1}^{\infty} m\left(f\left(\left[c_{k}, d_{k}\right]\right)=\sum_{k=1}^{\infty}\left(f\left(d_{k}\right)-f\left(c_{k}\right)\right)\right.
$$


Finally, given an integer $N>0$, then it must be that

$$
\left.\sum_{k=1}^{N}\left(d_{k}-c_{k}\right)<\sum_{k=1}^{\infty}\left(d_{k}-c_{k}\right) \leq \sum_{k=1}^{\infty}\left(b_{k}-a_{k}\right)\right)<\delta
$$

so that $\sum_{k=1}^{N}\left(f\left(d_{k}\right)-f\left(c_{k}\right)\right)<\epsilon . \quad N$ arbitrary then implies $m^{*}(f(E)) \leq$ $\sum_{k=1}^{\infty}\left(f\left(d_{k}\right)-f\left(c_{k}\right)\right) \leq \epsilon$. Thus, $m^{*}(f(E))=0$ since $\epsilon$ is arbitrary.

Assume now $E$ is a measurable subset of $[a, b]$.

By v of Proposition 2.15, there is a set $F$ that is the union of a countable collection of closed sets, $F \subseteq E$ with $m^{*}(E \backslash F)=0$, i.e., $E=K \cup\left(\cup_{n=1}^{\infty} F_{n}\right)$, where $m^{*}(K)=0, F=\cup_{n=1}^{\infty} F_{n}$, and $F_{n}$ is closed for each $n$, thus compact (Proposition 2.3). Note then that $f(E)=f\left(K \cup\left(\cup_{n=1}^{\infty} F_{n}\right)\right)=f(K) \cup$ $\left(\cup_{n=1}^{\infty} f\left(F_{n}\right)\right)$. Since $m(K)=0$, then $m(f(K))=0$ as just proved above. Thus, $f(K)$ is measurable. Also since $f$ is continuous and $F_{n}$ is compact for each $n$, it must be that $f\left(F_{n}\right)$ is compact for each $n$ [18] and thus measurable (Proposition 2.14). It then follows that $f(E)$ is the union of a countable collection of measurable sets and therefore it must be measurable.

Proposition 3.22 (Banach-Zarecki Theorem): Let $f$ be a real-valued function on $[a, b]$. Then $f$ is absolutely continuous on $[a, b]$ if and only if it satisfies the following three conditions:

i. $f$ is continuous on $[a, b]$.

ii. $f$ is of bounded variation on $[a, b]$.

iii. $f$ maps sets of measure zero to sets of measure zero.

Proof: The necessity was established in Observation 3.3, Proposition 3.10, and Proposition 3.21. For the sufficiency, assume $f$ satisfies all three conditions. Given $[c, d] \subseteq[a, b]$, we show

$$
|f(d)-f(c)| \leq \int_{c}^{d}\left|f^{\prime}(x)\right| d x
$$

By condition ii, $f$ is of bounded variation on $[a, b]$, so that by Corollary 3.3, $f$ is differentiable a.e. on $[a, b]$ and therefore on $[c, d]$. Accordingly, let $E_{1}$, $E_{2}$ be subsets of $[c, d],[c, d]=E_{1} \cup E_{2}, f^{\prime}$ exists on $E_{1}$ and $m\left(E_{2}\right)=0$. By condition iii, it then must be that $m\left(f\left(E_{2}\right)\right)=0$.

Assume without any loss of generality that $f(c)<f(d)$. By condition i, $f$ 
is continuous on $[a, b]$, so that by the intermediate value theorem [18], given $y, f(c)<y<f(d)$, there must be $x, c<x<d$, with $f(x)=y$. Thus, $[f(c), f(d)] \subseteq f([c, d])$, and since $f$ is measurable on $[a, b]$ ( $f$ is continuous on $[a, b])$ and $E_{1}$ is measurable $\left(E_{1}=[c, d] \backslash E_{2}\right)$, by Proposition 3.20, we get

$$
\begin{aligned}
|f(d)-f(c)| & \leq m(f([c, d]))=m\left(f\left(E_{1}\right) \cup f\left(E_{2}\right)\right) \\
& \leq m^{*}\left(f\left(E_{1}\right)\right)+m\left(f\left(E_{2}\right)\right)=m^{*}\left(f\left(E_{1}\right)\right)+0 \\
& =m^{*}\left(f\left(E_{1}\right)\right) \leq \int_{E_{1}}\left|f^{\prime}(x)\right| d x=\int_{c}^{d}\left|f^{\prime}(x)\right| d x .
\end{aligned}
$$

By Corollary 3.3, since $f$ is of bounded variation, it must be that $f^{\prime}$ is integrable over $[a, b]$ and so is $\left|f^{\prime}\right|$ by Proposition 2.31. Given $\epsilon>0$, by Proposition 2.38, there is $\delta>0$ such that if $A$ is a measurable set with $m(A)<\delta$, then $\int_{A}\left|f^{\prime}(x)\right| d x<\epsilon$. Accordingly, for any integer $n>0$ and any disjoint collection of open intervals $\left(x_{i}, x_{i}^{\prime}\right) \subseteq[a, b], i=1, \ldots, n$, with $\sum_{i=1}^{n}\left(x_{i}^{\prime}-x_{i}\right)<\delta$, let $A=\cup_{i=1}^{n}\left(x_{i}^{\prime}-x_{i}\right)$. Since $m(A)<\delta$, then

$$
\sum_{i=1}^{n}\left|f\left(x_{i}^{\prime}\right)-f\left(x_{i}\right)\right| \leq \sum_{i=1}^{n} \int_{x_{i}}^{x_{i}^{\prime}}\left|f^{\prime}(x)\right| d x=\int_{A}\left|f^{\prime}(x)\right| d x<\epsilon .
$$

Thus, $f$ is absolutely continuous.

Proposition 3.23 (Inverse function theorem): Let $f$ be a strictly monotonic continuous function on $[a, b]$. Then $I=f([a, b])$ is a closed interval with endpoints $f(a), f(b)$, and $f^{-1}$, the inverse function of $f$, exists on $I$, and is strictly monotonic and continuous on $I$. Given $x_{0} \in[a, b]$ such that $f$ is differentiable at $x_{0}$ with $f^{\prime}\left(x_{0}\right) \neq 0$, then $f^{-1}$ is differentiable at $y_{0}=f\left(x_{0}\right)$ with

$$
\left(f^{-1}\right)^{\prime}\left(y_{0}\right)=1 / f^{\prime}\left(x_{0}\right) .
$$

Proof: Without any loss of generality, assume $f$ is increasing on $[a, b]$. Since $f$ is strictly increasing and continuous, $f$ is one-to-one and by the intermediate value theorem [18], its range is $I=[f(a), f(b)]=f([a, b])$. Thus, $f^{-1}$ exists on $I$ and is strictly increasing from $I$ onto $[a, b]$. By Corollary 3.2, $f^{-1}$ is continuous on $I$. With $y_{0}=f\left(x_{0}\right), y=f(x)$, by the continuity of $f^{-1}$, if $y \rightarrow y_{0}$, it must be that $x \rightarrow x_{0}$. Thus,

$$
\frac{f^{-1}(y)-f^{-1}\left(y_{0}\right)}{y-y_{0}}=\frac{x-x_{0}}{f(x)-f\left(x_{0}\right)} \rightarrow 1 / f^{\prime}\left(x_{0}\right)
$$


as $y \rightarrow y_{0}$, since $f$ is differentiable at $x_{0}$, and $f^{\prime}\left(x_{0}\right) \neq 0$. Hence, $\left(f^{-1}\right)^{\prime}\left(y_{0}\right)$ exists and equals $1 / f^{\prime}\left(x_{0}\right)$.

Proposition 3.24 (Zarecki's criterion for an absolutely continuous inverse [5]): Let $f$ be a monotonic continuous function on $[a, b]$. Then $I=f([a, b])$ is a closed interval with endpoints $f(a), f(b)$, and $f^{-1}$ exists and is absolutely continuous on $I$ if and only if $\left\{x: f^{\prime}(x)=0\right\}$ has measure zero. Whenever $f^{-1}$ is absolutely continuous on the closed interval $I$, then

$$
\left(f^{-1}\right)^{\prime}=1 /\left(f^{\prime}\left(f^{-1}\right)\right) \text { a.e. on } I .
$$

Proof: Without any loss of generality, assume $f$ is increasing on $[a, b]$. If $f^{-1}$ exists or if $m^{*}\left(\left\{x: f^{\prime}(x)=0\right\}\right)=0$, then $f$ is strictly increasing. Thus, assume $f$ is strictly increasing. By Proposition 3.23, $I$ is closed, $I=[f(a), f(b)]$, and $f^{-1}$ exists on $I$ and is strictly increasing and continuous on $I$.

Assume $\left\{x: f^{\prime}(x)=0\right\}$ has measure zero. As already established, $f^{-1}$ is continuous on $I$, and since it is increasing, it is of bounded variation on $I$. Thus, by Proposition 3.22, it suffices to show that $f^{-1}$ maps sets of measure zero to sets of measure zero. For this purpose, let $E \subseteq I$ be of measure zero, and $F=f^{-1}(E)$ so that $f(F)=E$. Since $f$ is increasing, it is of bounded variation on $[a, b]$ as well, and by Corollary $3.12, f^{\prime}=0$ a.e. on $F$. Accordingly, let $F_{1}, F_{2}$ be subsets of $F, F=F_{1} \cup F_{2}, f^{\prime}=0$ on $F_{1}$ and $m\left(F_{2}\right)=0$. Since $F_{1} \subseteq\left\{x: f^{\prime}(x)=0\right\}$, then $m\left(F_{1}\right)=0$. Thus, $m(F)=0$.

Assume now $f^{-1}$ is absolutely continuous on $I$. By Corollary 3.8, since $f^{\prime}=0$ on $E=\left\{x: f^{\prime}(x)=0\right\}$, then $m(f(E))=0$. Thus, by Proposition 3.21, $m(E)=m\left(f^{-1}(f(E))\right)=0$.

Finally, whenever $f^{-1}$ is absolutely continuous on $I$, define $F_{1}, F_{2}, F_{3}$, disjoint subsets of $[a, b],[a, b]=F_{1} \cup F_{2} \cup F_{3}$, as follows. $F_{1}=\left\{x: f^{\prime}(x) \neq 0\right\}$, $F_{2}=\left\{x: f^{\prime}(x)=0\right\}, F_{3}=\left\{x: f^{\prime}(x)\right.$ does not exist $\}$. Since $f$ is of bounded variation on $[a, b], f$ is differentiable a.e. on $[a, b]$, thus, $m\left(F_{3}\right)=0$. Also, since $f^{-1}$ is absolutely continuous, then $m\left(F_{2}\right)=0$ as just proved above. Hence, since by Proposition 3.23, for each $x \in F_{1}, f^{-1}$ is differentiable at $y=f(x)$ with $\left(f^{-1}\right)^{\prime}(y)=1 / f^{\prime}(x)$, then $\left(f^{-1}\right)^{\prime}=1 /\left(f^{\prime}\left(f^{-1}\right)\right)$ a.e. on $I$.

Proposition 3.25 (Composition of absolutely continuous functions): Let $g$ be an absolutely continuous monotonic function from an interval $[a, b]$ into an interval $[c, d]$, and let $f$ be an absolutely continuous function on $[c, d]$. Then the function $h=f \circ g$ is absolutely continuous on $[a, b]$. 
Proof: Let $\epsilon>0$ be given. It follows easily that since $f$ is absolutely continuous, then there is $\rho>0$ such that for any integer $n>0$ and for any nonempty subset $A$ of $\{1, \ldots, n\}$ it must be that $\sum_{i \in A}\left|f\left(y_{i}^{\prime}\right)-f\left(y_{i}\right)\right|<\epsilon$ for disjoint open intervals $\left(y_{i}, y_{i}^{\prime}\right) \subseteq[c, d], i \in A$, with $\sum_{i \in A}\left(y_{i}^{\prime}-y_{i}\right)<\rho$. For $\rho$ as just described, since $g$ is also absolutely continuous, then there is $\delta>0$ such that for any integer $n>0$ it must be that $\sum_{i=1}^{n}\left|g\left(x_{i}^{\prime}\right)-g\left(x_{i}\right)\right|<\rho$ for disjoint open intervals $\left(x_{i}, x_{i}^{\prime}\right) \subseteq[a, b], i=1, \ldots, n$, with $\sum_{i=1}^{n}\left(x_{i}^{\prime}-x_{i}\right)<\delta$. Accordingly, for any integer $n>0$ let $\left(x_{i}, x_{i}^{\prime}\right) \subseteq[a, b], i=1, \ldots, n$, be any collection of disjoint open intervals with $\sum_{i=1}^{n}\left(x_{i}^{\prime}-x_{i}\right)<\delta$. Setting $A=\left\{i: 1 \leq i \leq n, g\left(x_{i}^{\prime}\right) \neq g\left(x_{i}\right)\right\}$, if $A \neq \emptyset$, then the collection of open intervals $\left(g\left(x_{i}\right), g\left(x_{i}^{\prime}\right)\right), i \in A$, if $g$ is increasing; $\left(g\left(x_{i}^{\prime}\right), g\left(x_{i}\right)\right), i \in A$, if $g$ is decreasing; must be pairwise disjoint by the monoticity of $g$ with $\sum_{i \in A}\left|g\left(x_{i}^{\prime}\right)-g\left(x_{i}\right)\right|<\rho$. Thus, if $A \neq \emptyset$, it must be that $S_{A}=\sum_{i \in A}\left|f\left(g\left(x_{i}^{\prime}\right)\right)-f\left(g\left(x_{i}\right)\right)\right|<\epsilon$. Setting $S_{A}=0$ if $A=\emptyset$, and since $\left|f\left(g\left(x_{i}^{\prime}\right)\right)-f\left(g\left(x_{i}\right)\right)\right|=0$ for $i \notin A$, then

$$
\sum_{i=1}^{n}\left|f\left(g\left(x_{i}^{\prime}\right)\right)-f\left(g\left(x_{i}\right)\right)\right|=S_{A}+0=S_{A}<\epsilon .
$$

Thus, $h=f \circ g$ is absolutely continuous on $[a, b]$.

Proposition 3.26 (Chain rule [22]): Given real-valued functions $F, f$ on $[c, d], F^{\prime}=f$ a.e. on $[c, d]$, and a function $u:[a, b] \rightarrow[c, d], u$ and $F \circ u$ differentiable a.e. on $[a, b]$, if $F$ maps zero-measure sets to zero-measure sets, then

$$
(F \circ u)^{\prime}=(f \circ u) u^{\prime} \text { a.e. on }[a, b] .
$$

Proof: Let $A=\left\{x \in[c, d]: F^{\prime}(x)=f(x)\right\}, B=[c, d] \backslash A$, and $C=$ $\{t \in[a, b]: u(t) \in B\}$. Clearly, $m B=0$. Letting $D=[a, b] \backslash C$, since $u$ is differentiable a.e. on $[a, b]$, then it is differentiable a.e. on $D$. Since $u(D) \subseteq A$ and $F$ is differentiable on $A$, the composite function $F \circ u$ is differentiable a.e. on $D$. Indeed it is differentiable exactly at the points in $D$ where $u$ is differentiable. Thus, by the usual chain rule of calculus, $(F \circ u)^{\prime}(t)=F^{\prime}(u(t)) u^{\prime}(t)=f(u(t)) u^{\prime}(t)$, for $t \in D$ at which $u^{\prime}$ exists, i.e., $(F \circ u)^{\prime}=(f \circ u) u^{\prime}$ a.e. on $D$.

Note that if $m C=0$, then the proof is complete.

Thus, assuming $m C \neq 0$, we show $(F \circ u)^{\prime}=(f \circ u) u^{\prime}$ a.e. on $C$. Note $f \circ u$ is defined on $C$. Since $u(C) \subseteq B$, then $m(u(C))=m B=0$, and since $u^{\prime}$ exists 
a.e. on $C$, then by Corollary $3.12, u^{\prime}=0$ a.e. on $C$ so that $(f \circ u) u^{\prime}=0$ a.e. on $C$. In addition, since $F$ maps zero-measure sets to zero-measure sets, then $m(F(u(C)))=0$, and since $(F \circ u)^{\prime}$ exists a.e. on $C$, again by Corollary 3.12, $(F \circ u)^{\prime}=0$ a.e. on $C$. Thus, $(F \circ u)^{\prime}=0=(f \circ u) u^{\prime}$ a.e. on $C$.

Observation 3.8: With $B$ the subset of $[c, d]$ of measure zero on which $F^{\prime} \neq$ $f$, and $C=\{t \in[a, b]: u(t) \in B\}$, then for the case $m C \neq 0$ in the proof of Proposition 3.26 above it was proved that $u^{\prime}=0$ a.e. on $C$, and since $f \circ u$ is defined on $C$, then $(f \circ u) u^{\prime}=0$ a.e. on $C$ as well. Since it was also proved that $(F \circ u)^{\prime}=0$ a.e. on $C$, then it was concluded that $(F \circ u)^{\prime}=0=(f \circ u) u^{\prime}$ a.e. on $C$. However, it can happen that when using the chain rule as described in Proposition 3.26 above and in Corollary 3.13 below, although $F^{\prime}=f$ on $[c, d] \backslash B, f$ may not be defined everywhere on $B$. Thus, $f \circ u$ may not be defined on $C$ as required in the proof of Proposition 3.26 above when $m C \neq 0$. However, what matters here is that both $u^{\prime}$ and $(F \circ u)^{\prime}$ are zero a.e. on $C$. Therefore, assuming $m C \neq 0$, when computing $(F \circ u)^{\prime}$ with the chain rule as suggested in Proposition 3.26 above and in Corollary 3.13 below, if $(F \circ u)^{\prime}$ is set to zero at any point in $[a, b]$ at which $u^{\prime}$ is zero (whether or not $f \circ u$ is defined there), and computed or left undefined according to the chain rule elsewhere, then $(F \circ u)^{\prime}$ so obtained will be correct a.e. on $[a, b]$. Accordingly, assuming $m C \neq 0$, one should keep in mind that if $(F \circ u)^{\prime}$ is not computed as just suggested, so that $(F \circ u)^{\prime}$ might be left undefined at points where $f \circ u$ is not defined although $u^{\prime}$ is zero, one could end up with $(F \circ u)^{\prime}$ not defined on a set of nonzero measure in $[a, b]$. Actually, instead of computing $(F \circ u)^{\prime}$ as just suggested, we do something simpler. Since, as mentioned above, what matters here is that both $u^{\prime}$ and $(F \circ u)^{\prime}$ are zero a.e. on $C$, without any loss of generality, we simply set $f$ equal to 1 at points in $B$ where it is not defined and proceed with the chain rule to compute $(F \circ u)^{\prime}$, as $f \circ u$ is then defined on $C$ so that $(f \circ u) u^{\prime}$ is zero at points in $C$ where $u^{\prime}$ is zero, thus zero a.e. on $C$. More precisely, we define a new function $\hat{f}$ on $[c, d]$ by setting $\hat{f}$ equal to $f$ at points in $[c, d]$ where $f$ exists, and to 1 where it does not. In what follows, we will refer to $\hat{f}$ as $f$ extended to all of $[c, d]$. This function is then defined everywhere in $[c, d]$, equals $f$ a.e. on $[c, d]$, and takes the place of $f$ in the chain rule although it is still called $f$ there. Finally, note that above when we say anything about computing $(F \circ u)^{\prime}$ with the chain rule, it is not $(F \circ u)^{\prime}$ that is necessarily computed but a function that happens to be equal to $(F \circ u)^{\prime}$ a.e. on $[a, b]$. 
Corollary 3.13 (Chain rule (Alternate form) [22]): Given an absolutely continuous function $F$ on $[c, d]$, and a real-valued function $f$ on $[c, d], F^{\prime}=f$ a.e. on $[c, d]$, if $u:[a, b] \rightarrow[c, d]$ is a function such that $u$ and $F \circ u$ are differentiable a.e. on $[a, b]$, then

$$
(F \circ u)^{\prime}=(f \circ u) u^{\prime} \text { a.e. on }[a, b] \text {. }
$$

Proof: From Proposition 3.26 and Proposition 3.21.

Proposition 3.27 (Change of variable for Lebesgue integral [22]): Given a function $f$, Lebesgue integrable over $[c, d]$, and a function $u:[a, b] \rightarrow$ $[c, d]$, differentiable a.e. on $[a, b]$, then the following two conditions are equivalent, where $F(x)=\int_{c}^{x} f(t) d t, x \in[c, d]$ :

i. $F \circ u$ is absolutely continuous on $[a, b]$.

ii. $(f \circ u) u^{\prime}$ is Lebesgue integrable over $[a, b]$ and for all $\alpha, \beta \in[a, b]$ it must be that

$$
\int_{u(\alpha)}^{u(\beta)} f(x) d x=\int_{\alpha}^{\beta} f(u(t)) u^{\prime}(t) d t,
$$

with $\int_{u(\alpha)}^{u(\beta)} f(x) d x=-\int_{u(\beta)}^{u(\alpha)} f(x) d x=-\int_{[u(\beta), u(\alpha)]} f(x) d x$ if $u(\beta)<u(\alpha)$, and $\int_{\alpha}^{\beta} f(u(t)) u^{\prime}(t) d t=-\int_{\beta}^{\alpha} f(u(t)) u^{\prime}(t) d t=-\int_{[\beta, \alpha]} f(u(t)) u^{\prime}(t) d t$ if $\beta<\alpha$.

\section{Proof:}

i $\Rightarrow$ ii:

Since $F$ is absolutely continuous on $[c, d]$ (Proposition 3.11), $F^{\prime}=f$ a.e. on $[c, d]$ (Proposition 3.7), $u$ is differentiable a.e. on $[a, b]$, and $F \circ u$, being absolutely continuous on $[a, b]$, must be differentiable a.e. on $[a, b]$ (Corollary 3.5), then by Corollary 3.13 (chain rule), $(F \circ u)^{\prime}=(f \circ u) u^{\prime}$ a.e. on $[a, b]$ (here and in the corollaries that follow, without any loss of generality, $f$ is interpreted as $f$ extended to all of $[c, d]$ (Observation 3.8 about the chain rule)). Note, by Corollary 3.5, since $F \circ u$ is absolutely continuous on $[a, b]$, then $(f \circ u) u^{\prime}$ is Lebesgue integrable over $[a, b]$, and by Corollary 3.6 (Fundamental Theorem of Lebesgue integral calculus), applied to the absolutely continuous function $F \circ u$, for all $\alpha, \beta \in[a, b]$ it must be that

$$
\begin{aligned}
\int_{\alpha}^{\beta} f(u(t)) u^{\prime}(t) d t & =\int_{\alpha}^{\beta}(F \circ u)^{\prime}(t) d t=(F \circ u)(\beta)-(F \circ u)(\alpha) \\
& =F(u(\beta))-F(u(\alpha))=\int_{u(\alpha)}^{u(\beta)} f(x) d x .
\end{aligned}
$$


$\mathrm{ii} \Rightarrow \mathrm{i}$ :

Since $(f \circ u) u^{\prime}$ is Lebesgue integrable over $[a, b]$, and, in particular, for $x \in$ $[a, b]$

$$
F(u(x))-F(u(a))=\int_{u(a)}^{u(x)} f(s) d s=\int_{a}^{x} f(u(t)) u^{\prime}(t) d t,
$$

by Proposition 3.12, $F \circ u=F(u)$ must be absolutely continuous on $[a, b]$.

Corollary 3.14 (Change of variable for Lebesgue integral (Alternate form I) [22]): Given a function $f$, Lebesgue integrable over $[c, d]$, and a function $u:[a, b] \rightarrow[c, d]$, monotonic and absolutely continuous on $[a, b]$, then $(f \circ u) u^{\prime}$ is Lebesgue integrable over $[a, b]$ and for all $\alpha, \beta \in[a, b]$ it must be that

$$
\int_{u(\alpha)}^{u(\beta)} f(x) d x=\int_{\alpha}^{\beta} f(u(t)) u^{\prime}(t) d t .
$$

Proof: Since $u$ is clearly differentiable a.e. on $[a, b]$, and $F, F(x)=\int_{c}^{x} f(t) d t$, $x \in[c, d]$, is absolutely continuous so that the composition $F \circ u$ is absolutely continuous on $[a, b]$ by Proposition 3.25, then by Proposition 3.27, $(f \circ u) u^{\prime}$ is Lebesgue integrable over $[a, b]$ and for all $\alpha, \beta \in[a, b]$ it must be that

$$
\int_{u(\alpha)}^{u(\beta)} f(x) d x=\int_{\alpha}^{\beta} f(u(t)) u^{\prime}(t) d t .
$$

Corollary 3.15 (Change of variable for Lebesgue integral (Alternate form II) [22]): Given a function $f$, bounded and measurable on $[c, d]$, and a function $u:[a, b] \rightarrow[c, d]$, absolutely continuous on $[a, b]$, then $(f \circ u) u^{\prime}$ is Lebesgue integrable over $[a, b]$ and for all $\alpha, \beta \in[a, b]$ it must be that

$$
\int_{u(\alpha)}^{u(\beta)} f(x) d x=\int_{\alpha}^{\beta} f(u(t)) u^{\prime}(t) d t .
$$

Proof: By Proposition 2.32, $f$ is Lebesgue integrable over $[c, d]$. Since $u$ is clearly differentiable a.e. on $[a, b]$, and by Observation 3.6, with $F(x)=$ $\int_{c}^{x} f(t) d t, x \in[c, d]$, it must be that $F \circ u$ is absolutely continuous on $[a, b]$, then by Proposition 3.27, $(f \circ u) u^{\prime}$ is Lebesgue integrable over $[a, b]$ and for all $\alpha, \beta \in[a, b]$ it must be that

$$
\int_{u(\alpha)}^{u(\beta)} f(x) d x=\int_{\alpha}^{\beta} f(u(t)) u^{\prime}(t) d t .
$$


Corollary 3.16 (Change of variable for Lebesgue integral over a measurable set [9]:) Given $A$, a measurable subset of $[0,1]$, and a function $\gamma:[0,1] \rightarrow[0,1], \gamma$ absolutely continuous on $[0,1], \dot{\gamma}>0$ a.e. on $[0,1]$, $\gamma(0)=0, \gamma(1)=1$, then $\gamma^{-1}$ exists and is absolutely continuous on $[0,1]$, and $\tilde{A}=\gamma^{-1}(A)$ is a measurable subset of $[0,1]$. Accordingly, given a function $f$, Lebesgue integrable over $[0,1]$, then $(f \circ \gamma) \dot{\gamma}$ is Lebesgue integrable over $\tilde{A}$ and

$$
\int_{A} f(x) d x=\int_{\tilde{A}} f(\gamma(t)) \dot{\gamma}(t) d t .
$$

Proof: Clearly $\gamma$ is strictly increasing and thus $\gamma^{-1}$ exists and is absolutely continuous on $[0,1]$ by Proposition 3.24. By Proposition 3.21, $\tilde{A}=\gamma^{-1}(A)$ is then a measurable subset of $[0,1]$.

Define $I_{A}:[0,1] \rightarrow \mathbf{R}$ by $I_{A}(t)=1$ if $t \in A, I_{A}(t)=0$ if $t \in[0,1] \backslash A$, and $I_{\tilde{A}}:[0,1] \rightarrow \mathbf{R}$ by $I_{\tilde{A}}(t)=1$ if $t \in \tilde{A}, I_{\tilde{A}}(t)=0$ if $t \in[0,1] \backslash \tilde{A}$.

Note $I_{\tilde{A}}=I_{A} \circ \gamma$.

Also note $I_{A} \cdot f$ is Lebesgue integrable over $[0,1]$, since it equals $f$ on $A$ and 0 on $[0,1] \backslash A$. It follows then by Corollary 3.14 that

$$
\int_{0}^{1} I_{A}(x) f(x) d x=\int_{\gamma(0)}^{\gamma(1)} I_{A}(x) f(x) d x=\int_{0}^{1} I_{A}(\gamma(t)) f(\gamma(t)) \dot{\gamma}(t) d t,
$$

i.e.,

$$
\int_{A} f(x) d x=\int_{0}^{1} I_{\tilde{A}}(t) f(\gamma(t)) \dot{\gamma}(t) d t=\int_{\tilde{A}} f(\gamma(t)) \dot{\gamma}(t) d t
$$

\section{Functional Data and Shape Analysis and its Connections to Lebesgue Integration and Absolute Continuity}

Observation 4.1: In what follows, we review some important aspects of functional data and shape analysis of the type in [23], while at the same time pointing out its dependence on Lesbesgue integration, absolute continuity and the connections between them. As in [23] where absolutely continuous functions on $[0,1]$ are generalized to functions of range $\mathbf{R}^{\mathbf{n}}, \mathbf{R}$ the set of real numbers, $n$ a positive integer, we consider absolutely continuous functions on $[0,1]$ but restrict ourselves to those with range in $\mathbf{R}^{1}=\mathbf{R}$. We denote by $A C[0,1]$ the set of such functions. With two absolutely continuous functions on $[0,1]$ considered equal if they differ by a constant, we note that the 
principal goal in [23] is essentially that of presenting tools for analyzing the shapes of absolutely continuous functions and defining a distance metric for computing a distance between any two of them. Specializing to $A C[0,1]$, a crucial aspect of the approach in [23] is then that of identifying a bijective correspondence between functions in $A C[0,1]$ and functions in $L^{2}[0,1]$, and taking advantage of this correspondence to compute easily the distance between functions in $A C[0,1]$ (the definition of this distance in terms of the so-called Fisher-Rao metric appears below) by computing the distance between the corresponding functions in $L^{2}[0,1]$. Actually, as mentioned above, the goal of this approach is not so much that of computing the distance between functions in $A C[0,1]$ but of computing the distance between their shapes. More precisely, in this approach, each function in $A C[0,1]$ is associated with its unique (a.e. on $[0,1]$ ) so-called square-root slope function (SRSF) in $L^{2}[0,1]$, and vice versa, and a distance metric is defined for computing the distance between the shapes of any two functions in $A C[0,1]$ in terms of the $L^{2}$ distances between SRSF's of reparametrizations of the two functions. This distance, although computed in $L^{2}[0,1]$, is a measure of how much one of the absolutely continuous functions must be reparametrized (with so-called warping functions) to align as much as possible with the other one. Since given two functions in $A C[0,1]$ that are not equal, the possibility exists that one function can be reparametrized to align exactly with the other one, i.e., become exactly the other one, the set of reparametrization functions or warping functions then induces a quotient space of $L^{2}[0,1]$. Accordingly, a distance metric is defined in [23] that computes the distance between any two equivalence classes in the quotient space of $L^{2}[0,1]$ by the set of warping functions, thus computing the distance between the shapes of the two corresponding functions in $A C[0,1]$.

Definition 4.1 (SRSF representation of functions [23]): Given $f \in$ $A C[0,1]$, the real-valued square-root slope function (SRSF) $q$ of $f$, is defined for each $t \in[0,1]$ at which $f^{\prime}$ exists by

$$
q(t)=\operatorname{sign}\left(f^{\prime}(t)\right) \sqrt{\left|f^{\prime}(t)\right|} .
$$

Observation 4.2: By Corollary 3.5, $f^{\prime}$ exists a.e. on $[0,1]$. Thus $q$ is defined a.e. on $[0,1]$. We note that $q$, the SRSF of $f$, is the 1 -dimensional version of the square-root velocity function (SRVF) $q$ of an absolutely continuous function $f, f:[0,1] \rightarrow \mathbf{R}^{n}$, defined as follows. Let $F: \mathbf{R}^{n} \rightarrow \mathbf{R}^{n}$ be the 
continuous map defined by $F(v)=v / \sqrt{|v|}$ if $|v| \neq 0, F(v)=0$ otherwise, $|\cdot|$ the Euclidean norm. Then the SRVF $q$ of $f, q:[0,1] \rightarrow \mathbf{R}^{n}$, is defined for each $t \in[0,1]$ at which $f^{\prime}$ exists by

$$
q(t)=F\left(f^{\prime}(t)\right)=f^{\prime}(t) / \sqrt{\left|f^{\prime}(t)\right|}
$$

if $\left|f^{\prime}(t)\right| \neq 0,0$ (in $\mathbf{R}^{n}$ ) otherwise.

See [10], [23] for a rigorous development of the SRVF.

Proposition 4.1 (Square integrability of SRSF [23]): Given $f \in$ $A C[0,1]$, the SRSF $q$ of $f$ is square-integrable over $[0,1]$, i.e., $q \in L^{2}[0,1]$, with $\int_{0}^{1}|q(t)|^{2} d t=\int_{0}^{1}\left|f^{\prime}(t)\right| d t$, i.e., $\|q\|_{2}^{2}=$ length of $f$.

Proof: By Corollary 3.5, $f^{\prime}$ is measurable and Lebesgue integrable over $[0,1]$. Note $h(t)=|q(t)|^{2}=\left|\operatorname{sign}\left(f^{\prime}(t)\right) \sqrt{\left|f^{\prime}(t)\right|^{2}}=\right| f^{\prime}(t) \mid$ for each $t \in[0,1]$ at which $f^{\prime}$ exists. Thus $h$ is measurable and Lebesgue integrable over $[0,1]$ (Proposition 2.31) so that $q \in L^{2}[0,1]$ and $\int_{0}^{1}|q(t)|^{2} d t=\int_{0}^{1}\left|f^{\prime}(t)\right| d t$.

Observation 4.3: As noted in Observation 2.21, a Lebesgue integrable function over a measurable set $E$ can be undefined on a subset of $E$ of measure zero. That can be the case above for functions $f^{\prime}$ and $|q|^{2}$ with $E=[0,1]$ which we know exist a.e. on $[0,1]$. However, without any loss of generality, in the spirit of Observation 3.8 about the chain rule, $f^{\prime}$ and $q$ will eventually be interpreted below as $f^{\prime}$ and $q$ extended to all of $[0,1]$. Finally, note the length of $f$ above is measured in $\mathbf{R}$, a 1 -dimensional space.

Proposition 4.2 (Reconstruction of an absolutely continuous function from its SRSF [23]): Given $f \in A C[0,1]$, let $q$ be the SRSF of $f$. Then for each $t \in[0,1]$ it must be that $f(t)=f(0)+\int_{0}^{t} q(s)|q(s)| d s$.

Proof: Note that for each $s \in[0,1]$ at which $f^{\prime}$ exists, then

$$
\begin{aligned}
q(s)|q(s)| & =\operatorname{sign}\left(f^{\prime}(s)\right) \sqrt{\left|f^{\prime}(s)\right|} \operatorname{sign}\left(f^{\prime}(s)\right) \sqrt{\left|f^{\prime}(s)\right| \mid} \\
& =\operatorname{sign}\left(f^{\prime}(s)\right) \sqrt{\left|f^{\prime}(s)\right|} \sqrt{\left|f^{\prime}(s)\right|} \\
& =\operatorname{sign}\left(f^{\prime}(s)\right)\left|f^{\prime}(s)\right|=f^{\prime}(s) .
\end{aligned}
$$

By Proposition 3.12, for each $t \in[0,1]$ it must be that $f(t)=f(0)+\int_{0}^{t} f^{\prime}(s) d s$. Thus, for each $t \in[0,1]$ it must be that $f(t)=f(0)+\int_{0}^{t} q(s)|q(s)| d s$. 
Proposition 4.3 ( $L^{2}[0,1]$ 's equivalence with the set of all SRSF's [23]): Let $q$ be in $L^{2}[0,1]$ and $C$ any real number. Let $h(t)=q(t)|q(t)|$ for each $t \in[0,1]$ at which $q$ exists. Then $h$ is defined a.e. on $[0,1], h$ is measurable and Lebesgue integrable over $[0,1]$, and the function $f$ defined for each $t \in[0,1]$ by $f(t)=C+\int_{0}^{t} h(s) d s$ is absolutely continuous on $[0,1]$ with $q$ equal to the SRSF of $f$ a.e. on $[0,1]$.

Proof: As $q$ is defined a.e. on $[0,1]$, then so is $h$. In addition, since $|h|=|q|^{2}$ is measurable and Lebesgue integrable over $[0,1]$, then so is $h$ (Proposition 2.31). By Proposition 3.12, $f$ is then absolutely continuous on $[0,1]$. Let $\hat{q}$ be the SRSF of $f$. Then for each $t \in[0,1]$ at which $f^{\prime}$ exists it must be that $\hat{q}(t)=\operatorname{sign}\left(f^{\prime}(t)\right) \sqrt{\left|f^{\prime}(t)\right|}$ and $\hat{q}$ is defined a.e. on $[0,1]$. Since by Proposition 3.7, $f^{\prime}=h$ a.e. on $[0,1]$, then it must also be that $\hat{q}(t)=\operatorname{sign}(h(t)) \sqrt{|h(t)|}$ for almost all $t \in[0,1]$.

$\operatorname{But} \operatorname{sign}(h(t)) \sqrt{|h(t)|}=\operatorname{sign}(q(t)|q(t)|) \sqrt{q(t)^{2}}=\operatorname{sign}(q(t))|q(t)|=q(t)$ for each $t \in[0,1]$ at which $q$ exists and therefore for almost all $t \in[0,1]$. Thus, $q=\hat{q}$ a.e. on $[0,1]$.

Definition 4.2: Under the composition of functions operation, the admissible class $\Gamma$ of warping functions is a semigroup of functions (not every element has an inverse) defined by

$$
\begin{gathered}
\Gamma=\{\gamma \mid \gamma:[0,1] \rightarrow[0,1], \gamma \text { absolutely continuous on }[0,1] \\
\dot{\gamma} \geq 0 \text { a.e. on }[0,1], \gamma(0)=0, \gamma(1)=1\}
\end{gathered}
$$

where $\dot{\gamma}$ is the derivative of $\gamma$.

The group $\Gamma_{0}$ of invertible warping functions, $\Gamma_{0} \subset \Gamma$, is defined by

$$
\Gamma_{0}=\{\gamma \mid \gamma \in \Gamma, \dot{\gamma}>0 \text { a.e. on }[0,1]\} \text {. }
$$

Observation 4.4: The functions in $\Gamma$ and $\Gamma_{0}$ play an important role in functional data and shape analysis as they are used to reparametrize an absolutely continuous function by warping its domain during the process of aligning its shape to the shape of another absolutely continuous function. As demonstrated in [10], [23], it is $\Gamma$ and $\Gamma_{0}$ that induce a quotient space 
of $L^{2}[0,1]$ with a well-defined distance metric. More on this below. We note, given $\gamma \in \Gamma$, since $\gamma$ is continuous, $\gamma(0)=0, \gamma(1)=1, \gamma:[0,1] \rightarrow[0,1]$, then by the intermediate value theorem $[18], \gamma([0,1])=[0,1]$. We note, given $\gamma \in \Gamma_{0}, \gamma$ is strictly increasing, thus has an inverse $\gamma^{-1}$ which is also in $\Gamma_{0}$ as $\gamma^{-1}(0)=0, \gamma^{-1}(1)=1, \gamma^{-1}$ is absolutely continuous on $[0,1]$ by Proposition 3.24, and $\left(\gamma^{-1}\right)^{\prime}>0$ a.e. on [0,1], also from Proposition 3.24, since $\gamma$ (the inverse of $\gamma^{-1}$ ) is absolutely continuous on $[0,1]$.

Note, given $\gamma, \tilde{\gamma} \in \Gamma$, then $\gamma \circ \tilde{\gamma}$ is absolutely continuous on $[0,1]$ by Proposition 3.25 and clearly $(\gamma \circ \tilde{\gamma})(0)=0,(\gamma \circ \tilde{\gamma})(1)=1$. Accordingly, if $\gamma, \tilde{\gamma} \in \Gamma_{0}$, in order to conclude that $\gamma \circ \tilde{\gamma} \in \Gamma_{0}$, we prove $(\gamma \circ \tilde{\gamma})^{\prime}>0$ a.e. on $[0,1]$. For this purpose let $A=\{t \in[0,1]: \dot{\gamma}(t)>0\}, B=[0,1] \backslash A, C=\tilde{\gamma}^{-1}(B)$. Clearly, $m B=0$ and since $\tilde{\gamma}^{-1}$ is absolutely continuous on $[0,1]$ as just proved above, then $m C=0$ by Proposition 3.21. Let $D=[0,1] \backslash C$. Accordingly, we only need to prove $(\gamma \circ \tilde{\gamma})^{\prime}>0$ a.e. on $D$. Clearly, $\dot{\tilde{\gamma}}$ exists (and is positive) a.e. on $D$. Since $\tilde{\gamma}(D) \subseteq A$, and $\dot{\gamma}$ exists (and is positive) on $A$, then $(\gamma \circ \tilde{\gamma})^{\prime}$ exists a.e. on $D$. Indeed it exists exactly at the points in $D$ where $\dot{\tilde{\gamma}}$ exists. Thus, by the usual chain rule of calculus, $(\gamma \circ \tilde{\gamma})^{\prime}(t)=\dot{\gamma}(\tilde{\gamma}(t)) \dot{\tilde{\gamma}}(t)$ for $t \in D$ at which $\dot{\tilde{\gamma}}$ exists. Since as mentioned above $\dot{\gamma}(\tilde{\gamma}(t))$ exists and is positive for all $t \in D$, and $\dot{\tilde{\gamma}}$ exists and is positive a.e. on $D$, then $(\gamma \circ \tilde{\gamma})^{\prime}=(\dot{\gamma} \circ \tilde{\gamma}) \dot{\tilde{\gamma}}>0$ a.e. on $D$.

Finally, given $\gamma, \tilde{\gamma} \in \Gamma$, we show $\gamma \circ \tilde{\gamma} \in \Gamma$. It suffices to show $(\gamma \circ \tilde{\gamma})^{\prime} \geq 0$ a.e. on $[0,1]$. For this purpose let $A=\{t \in[0,1]: \dot{\gamma}(t) \geq 0\}, B=[0,1] \backslash A$, $C=\{t \in[a, b]: \tilde{\gamma}(t) \in B\}$. Clearly, $m B=0$. Letting $G=[a, b] \backslash C$, then we can show that $(\gamma \circ \tilde{\gamma})^{\prime} \geq 0$ a.e. on $\mathrm{G}$, in the same manner we showed above for $\gamma, \tilde{\gamma} \in \Gamma_{0}$ that $(\gamma \circ \tilde{\gamma})^{\prime}>0$ a.e. on $D$. Thus, in order to complete the proof we show $(\gamma \circ \tilde{\gamma})^{\prime}=0$ a.e. on $C$. Since $\tilde{\gamma}(C) \subseteq B$, then $m(\tilde{\gamma}(C))=m B=0$, and since $\gamma$ is absolutely continuous on $[0,1]$, then $m(\gamma(\tilde{\gamma}(C)))=0$ by Proposition 3.21. Note $(\gamma \circ \tilde{\gamma})^{\prime}$ exists a.e. on $C$ as $\gamma \circ \tilde{\gamma}$ is absolutely continuous on $[0,1]$. That $(\gamma \circ \tilde{\gamma})^{\prime}=0$ a.e. on $C$ now follows follows from Corollary 3.12 .

Proposition 4.4 (SRSF of a warped absolutely continuous function [23]): Given $f \in A C[0,1]$ and $\gamma \in \Gamma$, then $f \circ \gamma \in A C[0,1]$ and $(f \circ \gamma)(0)=f(0)$. With $q$ the SRSF of $f$, without any loss of generality, in the spirit of Observation 3.8 about the chain rule, interpreting $f^{\prime}$ and $q$ as $f^{\prime}$ and $q$ extended to all of $[0,1]$, it then follows that the SRSF of $f \circ \gamma$ equals $(q \circ \gamma) \sqrt{\dot{\gamma}}$ a.e. on $[0,1]$. 
Proof: Clearly, $(f \circ \gamma)(0)=f(\gamma(0))=f(0)$. That $f \circ \gamma \in A C[0,1]$ follows directly from Proposition 3.25. Accordingly, it then follows from Corollary 3.13 (chain rule) that $(f \circ \gamma)^{\prime}=\left(f^{\prime} \circ \gamma\right) \dot{\gamma}$ a.e. on $[0,1]$. Thus, the SRSF of $f \circ \gamma$ which is defined for each $t \in[0,1]$ at which $(f \circ \gamma)^{\prime}$ exists as $\operatorname{sign}\left((f \circ \gamma)^{\prime}(t)\right) \sqrt{\left|(f \circ \gamma)^{\prime}(t)\right|}$ must equal

$$
\begin{aligned}
\operatorname{sign}\left(\left(f^{\prime} \circ \gamma\right)(t) \dot{\gamma}(t)\right) \sqrt{\left|\left(f^{\prime} \circ \gamma\right)(t) \dot{\gamma}(t)\right|} & =\operatorname{sign}\left(f^{\prime}(\gamma(t))\right) \sqrt{\left|f^{\prime}(\gamma(t))\right|} \sqrt{\dot{\gamma}(t)} \\
& =q(\gamma(t)) \sqrt{\dot{\gamma}(t)}
\end{aligned}
$$

for almost all $t \in[0,1]$.

Observation 4.5: Note the SRSF $q$ of $f$ is defined (Definition 4.1) for each $t \in[0,1]$ at which $f^{\prime}$ exists. However, although the SRSF of $f \circ \gamma$ equals $(q \circ \gamma) \sqrt{\dot{\gamma}}$ a.e. on $[0,1]$, it is not necessarily true that the SRSF of $f \circ \gamma$ exists at each $t \in[0,1]$ for which $q(\gamma(t)) \sqrt{\dot{\gamma}(t)}$ exists or if it exists it is equal to it.

Observation 4.6: An isometry is a distance-preserving transformation between two metric spaces. Here we describe in a nonrigorous manner isometries (and differentials as well) in the context of differential and Riemannian geometry. Let $M, N$ be spaces and let $\varphi$ be a mapping from $M$ into $N$, with $M, N, \varphi$ satisfying certain smoothness properties (in the language of differential geometry, $M$ and $N$ are smooth or differentiable manifolds which are spaces that locally resemble Euclidean, Hilbert or Banach spaces, and $\varphi$ is differentiable (generalized to smooth manifolds); here and in what follows, differentiability in the context of smooth manifolds is assumed to be of all orders). Given $\epsilon>0, p \in M$, assume $\alpha:(-\epsilon, \epsilon) \rightarrow M$ can be defined, $\alpha(0)=p, \alpha$ a curve in $M$, differentiable (generalized to smooth manifolds) so that $\alpha^{\prime}(0)$ makes sense. Then $\alpha^{\prime}(0)$ is considered to be a tangent vector to the curve $\alpha$ at $t=0$, and to $M$ at $p$. Accordingly, the set of all tangent vectors to $M$ at $p$ is called the tangent space of $M$ at $p$ and denoted by $T_{p} M$. Similarly, given $q \in N$, the set of all tangent vectors to $N$ at $q$ is called the tangent space of $N$ at $q$ and denoted by $T_{q} N$. With $\alpha$ as above, define $\beta:(-\epsilon, \epsilon) \rightarrow N$ by $\beta=\varphi \circ \alpha$. Then $\beta(0)=\varphi(p), \beta$ is a curve in $N$, and we assume it is differentiable (generalized to smooth manifolds) so that $\beta^{\prime}(0)$ makes sense and is then in $T_{\varphi(p)} N$. The mapping $d \varphi_{p}: T_{p} M \rightarrow T_{\varphi(p)} N$ given by $d \varphi_{p}\left(\alpha^{\prime}(0)\right)=\beta^{\prime}(0)$ is a linear mapping called the differential of $\varphi$ at $p$. Finally, assume there is a correspondence on $M$, 
smooth in some manner (see below), that associates to each point $p$ in $M$ an inner product $<,>_{p}$ on the tangent space $T_{p} M$. Similarly, assume there is a correspondence on $N$, smooth in the same manner, that associates to each point $q$ in $N$ an inner product $<,>_{q}$ on the tangent space $T_{q} N$. If $\varphi$ as above is bijective and satisfies certain smoothness properties (in the language of differential geometry, $\varphi$ is a diffeomorphism: $\varphi$ is bijective, and $\varphi$ and $\varphi^{-1}$ are differentiable (generalized to smooth manifolds)), then $\varphi$ is called an isometry if $\left\langle u, v>_{p}=<d \varphi_{p}(u), d \varphi_{p}(v)>_{\varphi(p)}\right.$, for all $p \in M$ and all $u, v \in T_{p} M$, where the inner product on the left is the one on $T_{p} M$ and the inner product on the right is the one on $T_{\varphi(p)} N$. On the other hand, if $\varphi$ is differentiable and satisfies $\left\langle u, v>_{p}=\left\langle d \varphi_{p}(u), d \varphi_{p}(v)>_{\varphi(p)}\right.\right.$, for all $p \in M$ and all $u, v \in T_{p} M$, but is not a diffeomorphism, then $\varphi$ is called a semi-isometry.

In the language of Riemannian geometry, the smooth correspondence above between points in a smooth manifold and inner products on tangent spaces of the space at the points is called a Riemannian metric or structure. Smooth manifolds equipped with such a structure are called Riemannian manifolds. Using the Riemannian structure, the length of a curve in a Riemannian manifold $M$ is computed as follows. Given $\alpha:[0,1] \rightarrow M$, a curve or path in $M$, differentiable (generalized to smooth manifolds) on [0,1] so that $\alpha^{\prime}(t)$ makes sense for $t \in[0,1]$ and is then in the tangent space $T_{\alpha(t)} M$, the length $L(\alpha)$ of the the path $\alpha$ is then given by

$$
L(\alpha)=\int_{0}^{1} \sqrt{<\alpha^{\prime}(t), \alpha^{\prime}(t)>_{\alpha(t)}} d t
$$

where $<,>_{\alpha(t)}$ is the inner product on the tangent space $T_{\alpha(t)} M$, and the smoothness of the Riemannian structure is such that $\sqrt{\left\langle\alpha^{\prime}(t), \alpha^{\prime}(t)\right\rangle_{\alpha(t)}}$, $t \in[0,1]$, is integrable over $[0,1]$ so that $L(\alpha)$ is well defined. In addition, given $p, q \in M$, the geodesic distance $d(p, q)$ between them is defined as the minimum of the lengths of all paths $\alpha$ in $M, \alpha:[0,1] \rightarrow M$, differentiable (generalized to smooth manifolds) with $\alpha(0)=p$ and $\alpha(1)=q$, i.e.,

$$
d(p, q)=\min _{\alpha:[0,1] \rightarrow M, \alpha \text { differentiable on }[0,1], \alpha(0)=p, \alpha(1)=q} L(\alpha) .
$$

If a path $\alpha$ exists such that $d(p, q)$ achieves its minimum at $\alpha$, then $\alpha$ is called a geodesic in $M$ between $p$ and $q$. We note that geodesics in Euclidean spaces and $L^{2}[0,1]$ are given by straight lines. Thus, for example, given $p$, 
$q$ in $L^{2}[0,1]$, then $\alpha:[0,1] \rightarrow L^{2}[0,1]$ defined by $\alpha(t)=(1-t) p+t q$ for $t \in[0,1]$, is the geodesic between $p$ and $q$ and the distance $d(p, q)$ is

$$
\int_{0}^{1}\left(\int_{0}^{1}|p(s)-q(s)|^{2} d s\right)^{1 / 2} d t=\int_{0}^{1}\|p-q\|_{2} d t=\|p-q\|_{2} .
$$

Finally, we note that with the distance $d(p, q)$ as defined above, it then follows that an isometry also as defined above is indeed a distance-preserving transformation. We show this in a nonrigorous manner. Given Riemannian manifolds $M, N$, let $\alpha:[0,1] \rightarrow M$ be a path from $p$ to $q$ in $M, \alpha(0)=p$, $\alpha(1)=q, \varphi: M \rightarrow N$ an isometry. Let $\beta=\varphi \circ \alpha$. Then $\beta:[0,1] \rightarrow N$, $\beta(0)=\varphi(p), \beta(1)=\varphi(q)$, and $\beta$ is a path from $\varphi(p)$ to $\varphi(q)$ in $N$. Since for any $t \in[0,1]$ the differential $d \varphi_{\alpha(t)}: T_{\alpha(t)} M \rightarrow T_{\varphi(\alpha(t))} N$ is given by $d \varphi_{\alpha(t)}\left(\alpha^{\prime}(t)\right)=\beta^{\prime}(t)$, then

$$
\begin{aligned}
L(\beta) & =\int_{0}^{1} \sqrt{<\beta^{\prime}(t), \beta^{\prime}(t)>_{\beta(t)}} d t \\
& =\int_{0}^{1} \sqrt{<d \varphi_{\alpha(t)}\left(\alpha^{\prime}(t)\right), d \varphi_{\alpha(t)}\left(\alpha^{\prime}(t)\right)>_{\varphi(\alpha(t))}} d t \\
& =\int_{0}^{1} \sqrt{<\alpha^{\prime}(t), \alpha^{\prime}(t)>_{\alpha(t)}} d t=L(\alpha)
\end{aligned}
$$

since $\varphi$ is an isometry. Similarly, given a path $\beta:[0,1] \rightarrow N$ from $\varphi(p)$ to $\varphi(q)$ in $N$, there is a path $\alpha:[0,1] \rightarrow M$ from $p$ to $q$ in $M$ with $L(\alpha)=L(\beta)$. Thus, $d(p, q)=d(\varphi(p), \varphi(q))$.

See [6], [7], [11], [12], [23] for a more rigorous development of the concepts of smooth manifolds, Riemannian manifolds, differentials, isometries, etc.

Observation 4.7: In what follows, given $q \in L^{2}[0,1], \gamma \in \Gamma$, we use $(q, \gamma)$ as short notation for $(q \circ \gamma) \sqrt{\dot{\gamma}}$. Here again, without any loss of generality, in the spirit of Observation 3.8 about the chain rule, $q$ is interpreted as $q$ extended to all of $[0,1]$. As it will be shown below, $(q, \gamma) \in L^{2}[0,1]$ so that without any loss of generality, again in the spirit of Observation 3.8 about the chain rule, $(q, \gamma)$ can be interpreted as $(q, \gamma)$ extended to all of $[0,1]$, and given $\bar{\gamma} \in \Gamma$, $((q, \gamma), \bar{\gamma})$ can be interpreted as $((q, \gamma), \bar{\gamma})$ extended to all of $[0,1]$.

Proposition 4.5: Given $q \in L^{2}[0,1]$ and $\gamma \in \Gamma$, then $(q, \gamma) \in L^{2}[0,1]$. In addition, given $\bar{\gamma} \in \Gamma$, then $((q, \gamma), \bar{\gamma})=(q, \gamma \circ \bar{\gamma})$ a.e. on $[0,1]$, and if $\gamma \in \Gamma_{0}$, then $\left((q, \gamma), \gamma^{-1}\right)=q$ a.e. on $[0,1]$. 
Proof: Let $h(t)=|q(t)|^{2}$ for each $t \in[0,1]$ at which $q$ exists. Then $h$ is Lebesgue integrable over $[0,1]$ and by Corollary 3.14, $(h \circ \gamma) \dot{\gamma}=|q \circ \gamma|^{2} \dot{\gamma}$ is Lebesgue integrable over $[0,1]$. Thus, $(q, \gamma)=(q \circ \gamma) \sqrt{\dot{\gamma}} \in L^{2}[0,1]$.

Now, if $\bar{\gamma} \in \Gamma$, then

$$
\begin{aligned}
((q, \gamma), \bar{\gamma}) & =((q \circ \gamma) \sqrt{\dot{\gamma}}, \bar{\gamma})=(((q \circ \gamma) \sqrt{\dot{\gamma}}) \circ \bar{\gamma}) \sqrt{\dot{\bar{\gamma}}} \\
& =((q \circ \gamma \circ \bar{\gamma}) \sqrt{\dot{\gamma} \circ \bar{\gamma}}) \sqrt{\dot{\bar{\gamma}}}=(q \circ(\gamma \circ \bar{\gamma})) \sqrt{(\dot{\gamma} \circ \bar{\gamma}) \dot{\bar{\gamma}}} \\
& =(q \circ(\gamma \circ \bar{\gamma})) \sqrt{(\gamma \circ \bar{\gamma})^{\prime}}=(q, \gamma \circ \bar{\gamma})
\end{aligned}
$$

a.e. on $[0,1]$ using Corollary 3.13 (chain rule) as $\gamma \circ \bar{\gamma} \in A C[0,1]$, by interpreting $\dot{\gamma}$ as $\dot{\gamma}$ extended to all of $[0,1]$ (Observation 3.8 about the chain rule). Finally, if $\gamma \in \Gamma_{0}$, then

$$
\begin{aligned}
\left((q, \gamma), \gamma^{-1}\right) & =\left((q \circ \gamma) \sqrt{\dot{\gamma}}, \gamma^{-1}\right)=\left(((q \circ \gamma) \sqrt{\dot{\gamma}}) \circ \gamma^{-1}\right) \sqrt{\left(\gamma^{-1}\right)^{\prime}} \\
& =\left(\left(q \circ \gamma \circ \gamma^{-1}\right) \sqrt{\dot{\gamma} \circ \gamma^{-1}}\right) \sqrt{\left(\gamma^{-1}\right)^{\prime}}=q \sqrt{\left(\dot{\gamma} \circ \gamma^{-1}\right)\left(\gamma^{-1}\right)^{\prime}} \\
& =q \sqrt{\left(\dot{\gamma} \circ \gamma^{-1}\right) /\left(\dot{\gamma} \circ \gamma^{-1}\right)}=q
\end{aligned}
$$

a.e. on $[0,1]$ using Proposition 3.24 .

Definition 4.3: The action of $\Gamma_{0}$ on $L^{2}[0,1]$ is the operation that takes any element $\gamma \in \Gamma_{0}$ and any element $q$ of $L^{2}[0,1]$, and computes $(q, \gamma)=(q \circ \gamma) \sqrt{\dot{\gamma}}$. The action of $\Gamma$ on $L^{2}[0,1]$ is similarly defined.

Proposition 4.6 (Action of $\Gamma$ on $L^{2}[0,1]$ is by semi-isometries. Action of $\Gamma_{0}$ on $L^{2}[0,1]$ is by isometries [23]): For each $\gamma \in \Gamma$, let $\varphi^{\gamma}: L^{2}[0,1] \rightarrow$ $L^{2}[0,1]$ be defined for $q \in L^{2}[0,1]$ by $\varphi^{\gamma}(q)=(q, \gamma)=(q \circ \gamma) \sqrt{\gamma}$. Then $\varphi^{\gamma}$ is differentiable and

$$
<d \varphi^{\gamma}(u), d \varphi^{\gamma}(v)>=<(u, \gamma),(v, \gamma)>=<u, v>
$$

for any $u, v \in L^{2}[0,1]$, where $<,>$ is the $L^{2}[0,1]$ inner product and $d \varphi^{\gamma}$ is the differential of $\varphi^{\gamma}$, with $<,>$ and $d \varphi^{\gamma}$ the same at every $q \in L^{2}[0,1]$. Thus, $\varphi^{\gamma}$ is a semi-isometry and the action of $\Gamma$ on $L^{2}[0,1]$ is said to be by semiisometries. If $\gamma \in \Gamma_{0}$, then $\varphi^{\gamma}$ is a diffeomorphism. Thus, $\varphi^{\gamma}$ is an isometry and the action of $\Gamma_{0}$ on $L^{2}[0,1]$ is said to be by isometries. 
Proof: If $\gamma \in \Gamma$, from Proposition 4.5 it follows that the range of $\varphi^{\gamma}$ is indeed in $L^{2}[0,1]$. Since the tangent space of $L^{2}[0,1]$ at any point is $L^{2}[0,1]$ itself, it follows that $<,>$ is the same at every $q \in L^{2}[0,1]$. Given $u, v \in L^{2}[0,1]$, from Proposition 2.41 (Hölder's inequality), $u \cdot v \in L^{1}$. Let $h(s)=u(s) v(s)$ for each $s \in[0,1]$ at which $u$ and $v$ exist. By Corollary 3.14 (change of variable),

$$
\begin{aligned}
<u, v> & =\int_{0}^{1} u(s) v(s) d s=\int_{\gamma(0)}^{\gamma(1)} h(s) d s=\int_{0}^{1} h(\gamma(t)) \dot{\gamma}(t) d t \\
& =\int_{0}^{1} u(\gamma(t)) \sqrt{\dot{\gamma}(t)} v(\gamma(t)) \sqrt{\dot{\gamma}(t)} d t \\
& =<(u, \gamma),(v, \gamma)>=<d \varphi^{\gamma}(u), d \varphi^{\gamma}(v)>
\end{aligned}
$$

as $\varphi^{\gamma}$ is linear so that it is differentiable and $d \varphi^{\gamma}$ acts on an element of $L^{2}[0,1]$ the same way $\varphi^{\gamma}$ does. Thus, $d \varphi^{\gamma}$ is the same at every $q \in L^{2}[0,1]$, and in addition, $\varphi^{\gamma}$ is a semi-isometry and the action of $\Gamma$ on $L^{2}[0,1]$ is by semi-isometries. If $\gamma \in \Gamma_{0}$, then $\varphi^{\gamma}$ is a bijection and its inverse $\varphi^{\gamma^{-1}}$ is linear so that it is differentiable. Thus, $\varphi^{\gamma}$ is a diffeomorphism and therefore an isometry, and the action of $\Gamma_{0}$ on $L^{2}[0,1]$ is by isometries.

Corollary 4.1 (Action of $\Gamma_{0}$ on $L^{2}[0,1]$ is distance preserving [23]): Given $q_{1}, q_{2} \in L^{2}$, and $\gamma \in \Gamma_{0}$, then $\left\|q_{1}-q_{2}\right\|_{2}=\left\|\left(q_{1}, \gamma\right)-\left(q_{2}, \gamma\right)\right\|_{2}$.

Proof: $d\left(q_{1}, q_{2}\right)=\left\|q_{1}-q_{2}\right\|_{2}$ and $d\left(\left(q_{1}, \gamma\right),\left(q_{2}, \gamma\right)\right)=\left\|\left(q_{1}, \gamma\right)-\left(q_{2}, \gamma\right)\right\|_{2}$ (Observation 4.6). The action of $\Gamma_{0}$ on $L^{2}[0,1]$ is by isometries (Proposition 4.6). Thus, $d\left(q_{1}, q_{2}\right)=d\left(\left(q_{1}, \gamma\right),\left(q_{2}, \gamma\right)\right)$ (Observation 4.6), and hence, $\left\|q_{1}-q_{2}\right\|_{2}=\left\|\left(q_{1}, \gamma\right)-\left(q_{2}, \gamma\right)\right\|_{2}$.

Corollary 4.2 (Action of $\Gamma_{0}$ on $L^{2}[0,1]$ is norm preserving [23]): Given $q \in L^{2}$, and $\gamma \in \Gamma_{0}$, then $\|q\|_{2}=\|(q, \gamma)\|_{2}$.

Observation 4.8 (Action of $\Gamma$ on $L^{2}[0,1]$ is distance and norm preserving): Corollary 4.1 and Corollary 4.2 can be shown to hold for all of $\Gamma$ as follows. Given $q_{1}, q_{2} \in L^{2}$, and $\gamma \in \Gamma$, then $q_{1}-q_{2} \in L^{2}$ and by Corollary 3.14 (change of variable),

$$
\left\|\left(q_{1}, \gamma\right)-\left(q_{2}, \gamma\right)\right\|_{2}^{2}=\int_{0}^{1}\left|q_{1}(\gamma(t)) \sqrt{\dot{\gamma}(t)}-q_{2}(\gamma(t)) \sqrt{\dot{\gamma}(t)}\right|^{2} d t
$$




$$
\begin{aligned}
& =\int_{0}^{1}\left(q_{1}(\gamma(t))-q_{2}(\gamma(t))\right)^{2} \dot{\gamma}(t) d t \\
& =\int_{\gamma(0)}^{\gamma(1)}\left(q_{1}(s)-q_{2}(s)\right)^{2} d s=\int_{0}^{1}\left(q_{1}(s)-q_{2}(s)\right)^{2} d s \\
& =\left\|q_{1}-q_{2}\right\|_{2}^{2} .
\end{aligned}
$$

Definition 4.4: Let $A C^{0}[0,1]=\left\{f: f \in A C[0,1], f^{\prime}>0\right.$ a.e. on $\left.[0,1]\right\}$. The Fisher-Rao metric at any $f \in A C^{0}[0,1]$ is defined as the inner product

$$
\ll u, v \gg_{f}=\frac{1}{4} \int_{0}^{1} \dot{u}(t) \dot{v}(t) \frac{1}{f^{\prime}(t)} d t .
$$

for any $u, v \in T_{f} A C^{0}[0,1]$.

Observation 4.9: The integral in the definition of the Fisher-Rao metric at $f \in A C^{0}[0,1]$ is well defined as $u, v$ are functions on $[0,1]$ that are absolutely continuous [21], hence $\dot{u}, \dot{v}, f^{\prime}$ exist a.e. on $[0,1], f^{\prime}>0$ a.e. on $[0,1]$, thus $\dot{u} / \sqrt{f^{\prime}}, \dot{v} / \sqrt{f^{\prime}}$ exist a.e. on $[0,1]$ and are in $L^{2}[0,1]$ (see below), so that $\dot{u} \dot{v} / f^{\prime}$ is Lebesgue integrable over [0,1] by Proposition 2.41 (Hölder's inequality). In addition, this metric, as defined at elements of $A C^{0}[0,1]$, is known to have the behavior of a Riemannian metric [23]. In what follows, we assume $A C^{0}[0,1]$ is endowed with this metric.

Proposition 4.7: Given $f \in A C^{0}[0,1]$ and $\gamma \in \Gamma_{0}$, then $f \circ \gamma \in A C^{0}[0,1]$ and $(f \circ \gamma)(0)=f(0)$.

Proof: That $f \circ \gamma \in A C[0,1]$ and $(f \circ \gamma)(0)=f(0)$ was established in Proposition 4.4. Accordingly, in order to conclude that $f \circ \gamma \in A C^{0}[0,1]$ we prove $(f \circ \gamma)^{\prime}>0$ a.e. on $[0,1]$. For this purpose let $A=\left\{t \in[0,1]: f^{\prime}(t)>\right.$ $0\}, B=[0,1] \backslash A, C=\gamma^{-1}(B)$. Clearly, $m B=0$ and since $\gamma^{-1}$ is absolutely continuous on $[0,1]$ (Observation 4.4), then $m C=0$ by Proposition 3.21. Let $D=[0,1] \backslash C$. Accordingly, we only need to prove $(f \circ \gamma)^{\prime}>0$ a.e. on $D$. Clearly, $\dot{\gamma}$ exists (and is positive) a.e. on $D$. Since $\gamma(D) \subseteq A$, and $f^{\prime}$ exists (and is positive) on $A$, then $(f \circ \gamma)^{\prime}$ exists a.e. on $D$. Indeed it exists exactly at the points in $D$ where $\dot{\gamma}$ exists. Thus, by the usual chain rule of calculus, $(f \circ \gamma)^{\prime}(t)=f^{\prime}(\gamma(t)) \dot{\gamma}(t)$ for $t \in D$ at which $\dot{\gamma}$ exists. Since as mentioned above $f^{\prime}(\gamma(t))$ exists and is positive for all $t \in D$, and $\dot{\gamma}$ exists and is positive a.e. on $D$, then $(f \circ \gamma)^{\prime}=\left(f^{\prime} \circ \gamma\right) \dot{\gamma}>0$ a.e. on $D$. 
Definition 4.5: The action of $\Gamma_{0}$ on $A C^{0}[0,1]$ is the operation that takes any element $\gamma \in \Gamma_{0}$ and any element $f$ of $A C^{0}[0,1]$, and computes $f \circ \gamma$.

Observation 4.10: In what follows, two functions in $A C[0,1]$ are considered equal if they differ by a constant. Simpler yet, we assume all functions in $A C[0,1]$ have the same value at zero. Since by Proposition 4.4, if $f \in$ $A C[0,1], \gamma \in \Gamma$, then $f \circ \gamma \in A C[0,1]$ and $(f \circ \gamma)(0)=f(0)$, and since in addition the SRSF of $(f+C)$ is the same for any constant $C$, the latter is a reasonable assumption.

Proposition 4.8 (Action of $\Gamma_{0}$ on $A C^{0}[0,1]$ with Fisher-Rao metric is by isometries [23]): For each $\gamma \in \Gamma_{0}$, let $\varphi^{\gamma}: A C^{0}[0,1] \rightarrow A C^{0}[0,1]$ be defined for $f \in A C^{0}[0,1]$ by $\varphi^{\gamma}(f)=f \circ \gamma$. Then $\varphi^{\gamma}$ is a diffeomorphism and

$$
\ll d \varphi_{f}^{\gamma}(u), d \varphi_{f}^{\gamma}(v) \gg_{f \circ \gamma}=\ll u \circ \gamma, v \circ \gamma \gg_{f \circ \gamma}=\ll u, v \gg_{f}
$$

for any $u, v \in T_{f} A C^{0}[0,1]$, where $\ll, \gg_{f}$ is the inner product that defines the Fisher-Rao metric at $f, \ll, \gg_{f \circ \gamma}$ is the inner product that defines it at $f \circ \gamma$, and $d \varphi_{f}^{\gamma}$ is the differential of $\varphi^{\gamma}$ at $f$. Thus, $\varphi^{\gamma}$ is an isometry and the action of $\Gamma_{0}$ on $A C^{0}[0,1]$ is said to be by isometries.

Proof: If $\gamma \in \Gamma_{0}$, from Proposition 4.7, the range of $\varphi^{\gamma}$ is indeed in $A C^{0}[0,1]$. As noted in Observation 4.9, given $f \in A C^{0}[0,1], u, v \in T_{f} A C^{0}[0,1]$, then $u$, $v$ are functions on $[0,1]$ that are absolutely continuous [21], and $h=\dot{u} \dot{v} / f^{\prime}$ is Lebesgue integrable over [0,1]. By Corollary 3.14 (change of variable), Corollary 3.13 (chain rule) as $u \circ \gamma, v \circ \gamma, f \circ \gamma \in A C[0,1]$, by interpreting $h, \dot{u}, \dot{v}, f^{\prime}$ as $h, \dot{u}, \dot{v}, f^{\prime}$ extended to all of $[0,1]$ (Observation 3.8 about the chain rule), and noting that all denominators below are greater than zero a.e. on $[0,1]$ (Proposition 4.7 and its proof), then

$$
\begin{aligned}
\ll u, v \gg_{f} & =\frac{1}{4} \int_{0}^{1} \dot{u}(s) \dot{v}(s) \frac{1}{f^{\prime}(s)} d s=\frac{1}{4} \int_{\gamma(0)}^{\gamma(1)} h(s) d s \\
& =\frac{1}{4} \int_{0}^{1} h(\gamma(t)) \dot{\gamma}(t) d t=\frac{1}{4} \int_{0}^{1} \dot{u}(\gamma(t)) \dot{v}(\gamma(t)) \frac{1}{f^{\prime}(\gamma(t))} \dot{\gamma}(t) d t \\
& =\frac{1}{4} \int_{0}^{1} \dot{u}(\gamma(t)) \dot{\gamma}(t) \dot{v}(\gamma(t)) \dot{\gamma}(t) \frac{1}{f^{\prime}(\gamma(t)) \dot{\gamma}(t)} d t
\end{aligned}
$$




$$
\begin{aligned}
& =\frac{1}{4} \int_{0}^{1}(u(\gamma(t)))^{\prime}(v(\gamma(t)))^{\prime} \frac{1}{(f(\gamma(t)))^{\prime}} d t \\
& =\ll u \circ \gamma, v \circ \gamma \gg_{f \circ \gamma}=\ll d \varphi_{f}^{\gamma}(u), d \varphi_{f}^{\gamma}(v) \gg_{f \circ \gamma}
\end{aligned}
$$

as $\varphi^{\gamma}$ is linear so that it is differentiable and $d \varphi_{f}^{\gamma}$ acts on an element of $T_{f} A C^{0}[0,1]$ the same way $\varphi^{\gamma}$ does on an element of $A C^{0}[0,1]$. Since $\gamma \in \Gamma_{0}$, then $\varphi^{\gamma}$ is a bijection and its inverse $\varphi^{\gamma^{-1}}$ is linear so that it is differentiable. Thus, $\varphi^{\gamma}$ is a diffeomorphism and therefore an isometry, and the action of $\Gamma_{0}$ on $A C^{0}[0,1]$ is by isometries.

Proposition 4.9 (Fisher-Rao metric on $A C^{0}[0,1]$ under SRSF representation becomes $L^{2}[0,1]$ metric [23]): Given $f \in A C^{0}[0,1]$ and $q$ the SRSF of $f$, define a mapping $F: A C^{0}[0,1] \rightarrow L^{2}[0,1]$ by $F(f)=q=\sqrt{f^{\prime}}$. Then $F$ is differentiable, and for any $v \in T_{f} A C^{0}[0,1]$, it must be that $F_{f}^{*}(v)=\dot{v} /\left(2 \sqrt{f^{\prime}}\right) \in T_{q} L^{2}[0,1]=L^{2}[0,1]$, where $F_{f}^{*}$ is the differential of $F$ at $f$. Given $v_{1}, v_{2} \in T_{f} A C^{0}[0,1]$, then $<F_{f}^{*}\left(v_{1}\right), F_{f}^{*}\left(v_{2}\right)>=\ll v_{1}, v_{2} \gg_{f}$, where $<,>$ is the $L^{2}[0,1]$ inner product and $\ll, \gg_{f}$ is the inner product that defines the Fisher-Rao metric at $f$.

Proof: Let $L_{0}^{1}[0,1]=\left\{\hat{f}: \hat{f} \in L^{1}[0,1], \hat{f}>0\right.$ a.e. on $\left.[0,1]\right\}$.

Given $\hat{f} \in L_{0}^{1}[0,1]$, define a mapping $S: L_{0}^{1}[0,1] \rightarrow L^{2}[0,1]$ by $S(\hat{f})=\sqrt{\hat{f}}$. In addition, given $f \in A C^{0}[0,1]$, define a mapping (the derivative mapping) $D: A C^{0}[0,1] \rightarrow L_{0}^{1}[0,1]$ by $D(f)=f^{\prime}$.

With $F$ as defined above, then $F=S \circ D$.

Given $v \in T_{f} A C^{0}[0,1]$, then $D_{f}^{*}(v)=\dot{v} \in T_{f^{\prime}} L_{0}^{1}[0,1]$, where $D_{f}^{*}$ is the differential of $D$ at $f$, as $D$ is linear so that it is differentiable and $D_{f}^{*}$ acts on an element of $T_{f} A C^{0}[0,1]$ the same way $D$ acts on an element of $A C^{0}[0,1]$.

Let $s: \mathbf{R} \rightarrow \mathbf{R}$ be the mapping defined by $s(x)=\sqrt{x}, x \in \mathbf{R}, x>0$. Then $s$ is differentiable for $x>0$, and $s^{*}(y)=s^{\prime}(x) y=y /(2 \sqrt{x})$ for any $y \in \mathbf{R}$, where $s^{*}$ is the differential of $s$. From this, following closely the definition of the differential of a differentiable function [11,23], it then follows that $S$ is differentiable and given $w \in T_{\hat{f}} L_{0}^{1}[0,1]$, then $S_{\hat{f}}^{*}(w)=w /(2 \sqrt{\hat{f}}) \in T_{\sqrt{\hat{f}}} L^{2}[0,1]$, where $S_{\hat{f}}^{*}$ is the differential of $S$ at $\hat{f}$.

Thus, $F=S \circ D$ is differentiable and its differential $F_{f}^{*}: T_{f} A C^{0}[0,1] \rightarrow$ $T_{q} L^{2}[0,1]$ at $f \in A C^{0}[0,1]$ is $S_{D(f)}^{*} \circ D_{f}^{*}=S_{f^{\prime}}^{*} \circ D_{f}^{*}[11]$.

Accordingly, given $v \in T_{f} A C^{0}[0,1]$, then $F_{f}^{*}(v)=S_{f^{\prime}}^{*}\left(D_{f}^{*}(v)\right)=S_{f^{\prime}}^{*}(\dot{v})=$ $\dot{v} /\left(2 \sqrt{f^{\prime}}\right) \in T_{\sqrt{f^{\prime}}} L^{2}[0,1]=T_{q} L^{2}[0,1]=L^{2}[0,1]$. 
Finally, given $v_{1}, v_{2} \in T_{f} A C^{0}[0,1]$, then

$$
\begin{aligned}
<F_{f}^{*}\left(v_{1}\right), F_{f}^{*}\left(v_{2}\right)> & =<\dot{v}_{1} /\left(2 \sqrt{f^{\prime}}\right), \dot{v}_{2} /\left(2 \sqrt{f^{\prime}}\right)>=\frac{1}{4} \int_{0}^{1} \dot{v}_{1}(t) \dot{v}_{2}(t) \frac{1}{f^{\prime}(t)} d t \\
& =\ll v_{1}, v_{2} \gg_{f} .
\end{aligned}
$$

Observation 4.11 (Distance between functions in $A C[0,1]$ ): Given $f_{1}$, $f_{2} \in A C[0,1]$, let $q_{1}, q_{2}$ be the SRSF's of $f_{1}, f_{2}$, respectively. We note that computing the distance between $f_{1}$ and $f_{2}$ with the Fisher-Rao metric as defined above may not be possible as a path in $A C[0,1]$ from $f_{1}$ to $f_{2}$ might contain functions whose derivatives are not positive a.e. on $[0,1]$. Even if this was not the case, the minimization involved would be nontrivial. Accordingly, motivated by Proposition 4.9 above, the convention is to say that the Fisher-Rao distance between $f_{1}$ and $f_{2}$ is $d_{F R}\left(f_{1}, f_{2}\right)=\left\|q_{1}-q_{2}\right\|_{2}$, i.e., the $L^{2}$ distance between $q_{1}$ and $q_{2}$. In addition, since the geodesic from $q_{1}$ to $q_{2}$ is a straight line, given $s \in[0,1]$, then $q=(1-s) q_{1}+s q_{2}$ is a function in this geodesic, and by Proposition 4.3, a function $f \in A C[0,1]$ can be computed for each $t \in[0,1]$ by $f(t)=C+\int_{0}^{t} q(x)|q(x)| d x$, where $C=f_{1}(0)=f_{2}(0)$, with the SRSF of $f$ equal to $q$ a.e. on $[0,1]$. Doing this for enough functions on the straight line joining $q_{1}$ and $q_{2}$, a collection of functions can be obtained in $A C[0,1]$ that are then said to approximate a geodesic (based on the Fisher-Rao metric) from $f_{1}$ to $f_{2}$.

Definition 4.6: Given $q \in L^{2}[0,1]$, define the orbit $[q]_{\Gamma_{0}}$ of $q$ under $\Gamma_{0}$ by $[q]_{\Gamma_{0}}=\left\{\bar{q}: \bar{q}=(q, \gamma)=(q \circ \gamma) \sqrt{\dot{\gamma}}\right.$ a.e. on $[0,1]$, some $\left.\gamma \in \Gamma_{0}\right\}$. Denote by $c l\left([q]_{\Gamma_{0}}\right)$ the closure in $L^{2}[0,1]$ of $[q]_{\Gamma_{0}}$.

Observation 4.12: In what follows, given $q_{1}, q_{2} \in L^{2}[0,1], q_{1} \in\left[q_{2}\right]_{\Gamma_{0}}$ so that $q_{1}=\left(q_{2}, \gamma\right)$ a.e. on $[0,1]$ for some $\gamma \in \Gamma_{0}$, without any loss of generality we may simply say $q_{1}=\left(q_{2}, \gamma\right)$. Accordingly, given $q_{1}, q_{2} \in L^{2}[0,1]$, $q_{1} \in\left[q_{2}\right]_{\Gamma_{0}}$ so that $q_{1}=\left(q_{2}, \gamma\right)$ for some $\gamma \in \Gamma_{0}$, then it follows (Proposition 4.5) that $\left[q_{1}\right]_{\Gamma_{0}} \subseteq\left[q_{2}\right]_{\Gamma_{0}}$, and $q_{2}=\left(q_{1}, \gamma^{-1}\right)$ so that $\left[q_{2}\right]_{\Gamma_{0}} \subseteq\left[q_{1}\right]_{\Gamma_{0}}$ and thus $\left[q_{1}\right]_{\Gamma_{0}}=\left[q_{2}\right]_{\Gamma_{0}}$. Using similar arguments, given $q_{1}, q_{2} \in L^{2}[0,1]$, an equivalence relation $\sim$ can be defined and justified on $L^{2}[0,1]$ for which $q_{1} \sim q_{2}$ if $q_{1}$ and $q_{2}$ are in the same orbit under $\Gamma_{0}$. Accordingly, with this equivalence relation a quotient space is obtained which is the set of all orbits of elements of $L^{2}[0,1]$ under $\Gamma_{0}$ and which we denote by $L^{2}[0,1] / \Gamma_{0}$. An attempt then can be made as follows to define a distance function $d$ between 
elements of $L^{2}[0,1] / \Gamma_{0}$ that would make $L^{2}[0,1] / \Gamma_{0}$ a metric space. Given $q_{1}, q_{2} \in L^{2}[0,1]$, let

$$
\begin{aligned}
d\left(\left[q_{1}\right]_{\Gamma_{0}},\left[q_{2}\right]_{\Gamma_{0}}\right) & =\inf _{\gamma_{1}, \gamma_{2} \in \Gamma_{0}}\left\|\left(q_{1}, \gamma_{1}\right)-\left(q_{2}, \gamma_{2}\right)\right\|_{2} \\
& =\inf _{\gamma \in \Gamma_{0}}\left\|q_{1}-\left(q_{2}, \gamma\right)\right\|_{2}=\inf _{\gamma \in \Gamma_{0}}\left\|\left(q_{1}, \gamma\right)-q_{2}\right\|_{2},
\end{aligned}
$$

where the bottom equations follow from Corollary 4.1 (action of $\Gamma_{0}$ on $L^{2}[0,1]$ is distance preserving) again using Proposition 4.5 where appropriate. Of the properties that $d$ must satisfy to be a distance function all have been established [23] except one: $d\left(\left[q_{1}\right]_{\Gamma_{0}},\left[q_{2}\right]_{\Gamma_{0}}\right)=0$ if and only if $\left[q_{1}\right]_{\Gamma_{0}}=\left[q_{2}\right]_{\Gamma_{0}}$. Unfortunately, as demonstrated in [10], the orbits as defined are not closed in $L^{2}[0,1]$, which allows for examples with $\left[q_{1}\right]_{\Gamma_{0}} \neq\left[q_{2}\right]_{\Gamma_{0}}$ but $d\left(\left[q_{1}\right]_{\Gamma_{0}},\left[q_{2}\right]_{\Gamma_{0}}\right)=0$.

Proposition 4.10 ([10]): Given $q_{1}, q_{2} \in L^{2}[0,1]$, then $d\left(\left[q_{1}\right]_{\Gamma_{0}},\left[q_{2}\right]_{\Gamma_{0}}\right)=0$ if and only if $\operatorname{cl}\left(\left[q_{1}\right]_{\Gamma_{0}}\right)=\operatorname{cl}\left(\left[q_{2}\right]_{\Gamma_{0}}\right)$. In particular, if $q_{1} \in \operatorname{cl}\left(\left[q_{2}\right]_{\Gamma_{0}}\right)$ so that $d\left(\left[q_{1}\right]_{\Gamma_{0}},\left[q_{2}\right]_{\Gamma_{0}}\right)=0$, then $\operatorname{cl}\left(\left[q_{1}\right]_{\Gamma_{0}}\right)=\operatorname{cl}\left(\left[q_{2}\right]_{\Gamma_{0}}\right)$.

Proof: If $d\left(\left[q_{1}\right]_{\Gamma_{0}},\left[q_{2}\right]_{\Gamma_{0}}\right)=0$, fix $\bar{q}_{1} \in\left[q_{1}\right]_{\Gamma_{0}}$ and note $\left[\bar{q}_{1}\right]_{\Gamma_{0}}=\left[q_{1}\right]_{\Gamma_{0}}$ (Observation 4.12) so that $d\left(\left[\bar{q}_{1}\right]_{\Gamma_{0}},\left[q_{2}\right]_{\Gamma_{0}}\right)=d\left(\left[q_{1}\right]_{\Gamma_{0}},\left[q_{2}\right]_{\Gamma_{0}}\right)=0$. Then given integer $n>0$, there is $\gamma_{n} \in \Gamma_{0}$ such that $\left\|\bar{q}_{1}-\left(q_{2}, \gamma_{n}\right)\right\|_{2}<1 / n$. Thus, $\bar{q}_{1} \in \operatorname{cl}\left(\left[q_{2}\right]_{\Gamma_{0}}\right)$. Since $\bar{q}_{1}$ is arbitrary in $\left[q_{1}\right]_{\Gamma_{0}}$ then $\left[q_{1}\right]_{\Gamma_{0}} \subseteq \operatorname{cl}\left(\left[q_{2}\right]_{\Gamma_{0}}\right)$, thus $\operatorname{cl}\left(\left[q_{1}\right]_{\Gamma_{0}}\right) \subseteq$ $\operatorname{cl}\left(\left[q_{2}\right]_{\Gamma_{0}}\right)$. Similarly, $c l\left(\left[q_{2}\right]_{\Gamma_{0}}\right) \subseteq \operatorname{cl}\left(\left[q_{1}\right]_{\Gamma_{0}}\right)$, thus $c l\left(\left[q_{1}\right]_{\Gamma_{0}}\right)=\operatorname{cl}\left(\left[q_{2}\right]_{\Gamma_{0}}\right)$.

Assume $\operatorname{cl}\left(\left[q_{1}\right]_{\Gamma_{0}}\right)=\operatorname{cl}\left(\left[q_{2}\right]_{\Gamma_{0}}\right)$. Then, in particular, $q_{1} \in \operatorname{cl}\left(\left[q_{2}\right]_{\Gamma_{0}}\right)$ so that given integer $n>0$, there is $\gamma_{n} \in \Gamma_{0}$ with $\left\|q_{1}-\left(q_{2}, \gamma_{n}\right)\right\|_{2}<1 / n$. Thus, $d\left(\left[q_{1}\right]_{\Gamma_{0}},\left[q_{2}\right]_{\Gamma_{0}}\right)=\inf _{\gamma \in \Gamma_{0}}\left\|q_{1}-\left(q_{2}, \gamma\right)\right\|_{2}=0$.

Observation 4.13: Using arguments similar to those in the proof of Proposition 4.10 above, given $q_{1}, q_{2} \in L^{2}[0,1]$, an equivalence relation $\sim$ can be defined and justified on $L^{2}[0,1]$ for which $q_{1} \sim q_{2}$ if $q_{1}$ and $q_{2}$ are in the closure of the same orbit under $\Gamma_{0}$. Accordingly, with this equivalence relation a quotient space is obtained which is the set of all closures of orbits of elements of $L^{2}[0,1]$ under $\Gamma_{0}$ and which we denote by $L^{2}[0,1] / \sim$. In what follows, we extend the function $d$ above to the quotient space $L^{2}[0,1] / \sim$.

Corollary 4.3 (Distance between equivalence classes in $L^{2}[0,1] / \sim$ [10], [23]): Given $q_{1}, q_{2} \in L^{2}[0,1]$, let

$$
d\left(c l\left(\left[q_{1}\right]_{\Gamma_{0}}\right), \operatorname{cl}\left(\left[q_{2}\right]_{\Gamma_{0}}\right)\right)=\inf _{\bar{q}_{1} \in \operatorname{cl}\left(\left[q_{1}\right]_{\Gamma_{0}}\right), \bar{q}_{2} \in c l\left(\left[q_{2}\right]_{\Gamma_{0}}\right)}\left\|\bar{q}_{1}-\bar{q}_{2}\right\|_{2} .
$$


Then $d\left(\operatorname{cl}\left(\left[q_{1}\right]_{\Gamma_{0}}\right), \operatorname{cl}\left(\left[q_{2}\right]_{\Gamma_{0}}\right)\right)=\inf _{\gamma_{1}, \gamma_{2} \in \Gamma_{0}}\left\|\left(q_{1}, \gamma_{1}\right)-\left(q_{2}, \gamma_{2}\right)\right\|_{2}=\inf _{\gamma \in \Gamma_{0}} \| q_{1}-$ $\left(q_{2}, \gamma\right)\left\|_{2}=\inf _{\gamma \in \Gamma_{0}}\right\|\left(q_{1}, \gamma\right)-q_{2} \|_{2}=d\left(\left[q_{1}\right]_{\Gamma_{0}},\left[q_{2}\right]_{\Gamma_{0}}\right)$, and $d$ is a distance function between elements of $L^{2}[0,1] / \sim$, so that $L^{2}[0,1] / \sim$ is a metric space with this distance function.

Proof: Note

$$
\inf _{\bar{q}_{1} \in c l\left(\left[q_{1}\right]_{\Gamma_{0}}\right), \bar{q}_{2} \in c l\left(\left[q_{2}\right]_{\Gamma_{0}}\right)}\left\|\bar{q}_{1}-\bar{q}_{2}\right\|_{2}=\inf _{\gamma_{1}, \gamma_{2} \in \Gamma_{0}}\left\|\left(q_{1}, \gamma_{1}\right)-\left(q_{2}, \gamma_{2}\right)\right\|_{2} .
$$

Thus, $d\left(c l\left(\left[q_{1}\right]_{\Gamma_{0}}\right), \operatorname{cl}\left(\left[q_{2}\right]_{\Gamma_{0}}\right)\right)=\inf _{\gamma_{1}, \gamma_{2} \in \Gamma_{0}}\left\|\left(q_{1}, \gamma_{1}\right)-\left(q_{2}, \gamma_{2}\right)\right\|_{2}=\inf _{\gamma \in \Gamma_{0}} \| q_{1}-$ $\left(q_{2}, \gamma\right)\left\|_{2}=\inf _{\gamma \in \Gamma_{0}}\right\|\left(q_{1}, \gamma\right)-q_{2} \|_{2}=d\left(\left[q_{1}\right]_{\Gamma_{0}},\left[q_{2}\right]_{\Gamma_{0}}\right)$, as previously noted in Observation 4.12 .

That $d$ is a distance function follows from Proposition 4.10 and results about properties of this distance function in [23].

Observation 4.14: Given $f_{1}, f_{2} \in A C[0,1]$, and $q_{1}, q_{2}$, the SRSF's of $f_{1}$, $f_{2}$, respectively, we note that $q_{1}$ and $q_{2}$ remain unchanged after translations of $f_{1}$ and $f_{2}$ (by translations we mean $f_{1}$ and $f_{2}$ become $f_{1}+c_{1}$ and $f_{2}+c_{2}$, respectively, for constants $c_{1}, c_{2}$ ) so that the distance between the equivalence classes of $q_{1}$ and $q_{2}$, defined by $d$ above, is the same before and after the translations. That this is true follows from the definition of the SRSF. For scalar multiplications of $f_{1}$ and $f_{2}$, the distance between the equivalence classes of $q_{1}$ and $q_{2}$ before and after the scalar multiplications can be approximated or computed exactly, if possible, by the same elements of $\Gamma_{0}$ as the following proposition shows. Accordingly, it is customary to normalize $q_{1}$ and $q_{2}$ so that $\left\|q_{1}\right\|_{2}=\left\|q_{2}\right\|_{2}=1$ and then compute the distance between their equivalence classes with $d$ as above, as from the comments just made doing so is compatible with the requirement that the shapes of $f_{1}$ and $f_{2}$ be invariant under translation and scalar multiplication.

Proposition 4.11 ([23]): Given $q_{1}, q_{2} \in L^{2}[0,1]$, and $\gamma^{*}, \gamma \in \Gamma_{0}$ for which $\left\|q_{1}-\left(q_{2}, \gamma^{*}\right)\right\|_{2} \leq\left\|q_{1}-\left(q_{2}, \gamma\right)\right\|_{2}$, then $\left\|b q_{1}-\left(c q_{2}, \gamma^{*}\right)\right\|_{2} \leq\left\|b q_{1}-\left(c q_{2}, \gamma\right)\right\|_{2}$, for any $b, c, b c>0$.

Proof: With $<,>$ as the $L^{2}$ inner product, note

$$
\left\|q_{1}-\left(q_{2}, \gamma^{*}\right)\right\|_{2}^{2}=\left\|q_{1}\right\|_{2}^{2}-2<q_{1},\left(q_{2}, \gamma^{*}\right)>+\left\|\left(q_{2}, \gamma^{*}\right)\right\|_{2}^{2},
$$



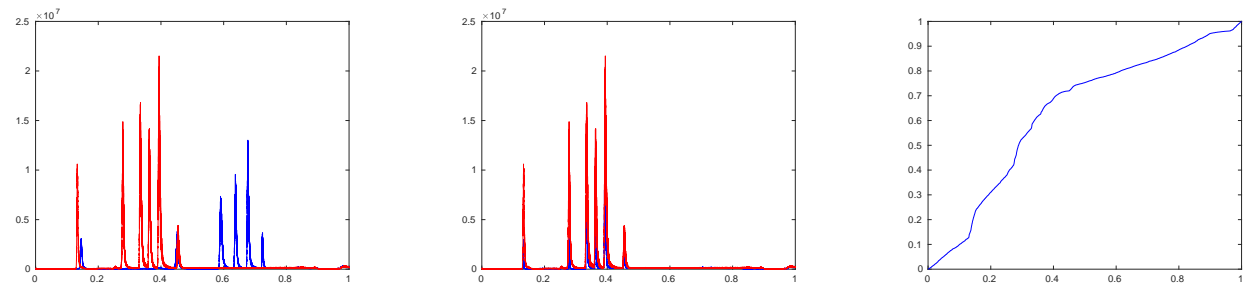

Figure 1: Function alignment by warping that is obtained from the computation of $d\left(c l\left(\left[q_{1}\right]_{\Gamma_{0}}\right), c l\left(\left[q_{2}\right]_{\Gamma_{0}}\right)\right)$.

and

$$
\left\|q_{1}-(q 2, \gamma)\right\|_{2}^{2}=\left\|q_{1}\right\|_{2}^{2}-2<q_{1},\left(q_{2}, \gamma\right)>+\left\|\left(q_{2}, \gamma\right)\right\|_{2}^{2} .
$$

Thus, $\left\|q_{1}-\left(q_{2}, \gamma^{*}\right)\right\|_{2} \leq\left\|q_{1}-\left(q_{2}, \gamma\right)\right\|_{2}$ and $\left\|q_{2}\right\|_{2}=\left\|\left(q_{2}, \gamma^{*}\right)\right\|_{2}=\left\|\left(q_{2}, \gamma\right)\right\|_{2}$, $b c>0$, imply $-2 b c<q_{1},\left(q_{2}, \gamma^{*}\right)>\leq-2 b c<q_{1},\left(q_{2}, \gamma\right)>$.

Accordingly, since $\left\|c q_{2}\right\|_{2}=\left\|\left(c q_{2}, \gamma^{*}\right)\right\|_{2}=\left\|\left(c q_{2}, \gamma\right)\right\|_{2}$, then

$$
\begin{array}{r}
\left\|b q_{1}\right\|_{2}^{2}-2<b q_{1},\left(c q_{2}, \gamma^{*}\right)>+\left\|\left(c q_{2}, \gamma^{*}\right)\right\|_{2}^{2} \\
\leq \quad\left\|b q_{1}\right\|_{2}^{2}-2<b q_{1},\left(c q_{2}, \gamma\right)>+\left\|\left(c q_{2}, \gamma\right)\right\|_{2}^{2},
\end{array}
$$

so that $\left\|b q_{1}-\left(c q_{2}, \gamma^{*}\right)\right\|_{2} \leq\left\|b q_{1}-\left(c q_{2}, \gamma\right)\right\|_{2}$.

Observation 4.15: Figure 1 illustrates an instance of approximately computing $d\left(c l\left(\left[q_{1}\right]_{\Gamma_{0}}\right), c l\left(\left[q_{2}\right]_{\Gamma_{0}}\right)\right)$ as expressed in Corollary 4.3 above. Here $q_{1}$, $q_{2}$ are the SRSF's of functions $f_{1}, f_{2}$, respectively, plotted in the lefmost diagram, $f_{1}$ in red, $f_{2}$ in blue, $q_{1}, q_{2}$ normalized so that $\left\|q_{1}\right\|_{2}=\left\|q_{2}\right\|_{2}=$ 1. The distance (about 0.1436) was approximately computed (in about 154 seconds) with adapt-DP [2], a fast linear Dynamic Programming algorithm. The resulting warping function $\gamma \in \Gamma_{0}$ that approximately minimizes $\left\|q_{1}-\left(q_{2}, \gamma\right)\right\|_{2}$ is plotted in the rightmost diagram, and $f_{1}$ and $f_{2} \circ \gamma$ are plotted in the middle diagram in which they appear essentially aligned. The functions $f_{1}$ and $f_{2}$ were given in the form of sets of 19,693 and 19,763 points, respectively, with nonuniform domains in $[0,1]$. A copy of adapt$D P$ with usage instructions and data files for the same example in Figure 1 can be obtained using links: https://doi.org/10.18434/T4/1502501 http://math.nist.gov/ JBernal /Fast_Dynamic_Programming.zip 
Definition 4.7: Given $q \in L^{2}[0,1]$, define the orbit $[q]_{\Gamma}$ of $q$ under $\Gamma$ by $[q]_{\Gamma}=\{\bar{q}: \bar{q}=(q, \gamma)=(q \circ \gamma) \sqrt{\dot{\gamma}}$ a.e. on $[0,1]$, some $\gamma \in \Gamma\}$.

Observation 4.16: In what follows, we present results found mostly in [10] for the purpose of showing that given $q \in L^{2}[0,1]$, then there exist $w \in$ $L^{2}[0,1], \gamma \in \Gamma$, such that $q=(w, \gamma),|w|$ constant a.e. on $[0,1], \operatorname{cl}\left([w]_{\Gamma_{0}}\right)=$ $[w]_{\Gamma}$ so that $q \in \operatorname{cl}\left([w]_{\Gamma_{0}}\right)$, and thus $\operatorname{cl}\left([q]_{\Gamma_{0}}\right)=\operatorname{cl}\left([w]_{\Gamma_{0}}\right)=[w]_{\Gamma}$. We note that this result doesn't change how $d\left(\operatorname{cl}\left(\left[q_{1}\right]_{\Gamma_{0}}\right), \operatorname{cl}\left(\left[q_{2}\right]_{\Gamma_{0}}\right)\right)$ in Corollary 4.3 is computed for $q_{1}, q_{2} \in L^{2}[0,1]$. It should still be done by computing $\inf _{\gamma \in \Gamma_{0}}\left\|q_{1}-\left(q_{2}, \gamma\right)\right\|_{2}$ or $\inf _{\gamma \in \Gamma_{0}}\left\|\left(q_{1}, \gamma\right)-q_{2}\right\|_{2}$ as implied by Corollary 4.3.

Proposition 4.12: $A_{0}=\left\{q \in L^{2}[0,1]:\|q\|_{2}=1, q>0\right.$ a.e. on $\left.[0,1]\right\}$ has closure in $L^{2}[0,1]$ equal to $A=\left\{q \in L^{2}[0,1]:\|q\|_{2}=1, q \geq 0\right.$ a.e. on $\left.[0,1]\right\}$.

Proof: Clearly $A_{0} \subset A$. Let $\epsilon>0$ be given. Given $q \in A \backslash A_{0}$, then a measurable subset $B$ of $[0,1]$ exists, $m(B)>0$, on which $q=0$. Let $C=[0,1] \backslash B$. Then $q>0$ a.e. on $C$ and $\int_{C} q(t)^{2} d t=\int_{0}^{1} q(t)^{2} d t=1$.

Choose $b>0, b<1$ with $1-b<\epsilon / 2$, and set $a=\left(\left(1-b^{2}\right) / m(B)\right)^{1 / 2}$.

Define a function $\hat{q}$ on $[0,1]$ by $\hat{q}=b q$ on $C$ and $\hat{q}=a$ on $B$. Then $\hat{q}>0$ a.e. on $[0,1]$ and $\int_{0}^{1} \hat{q}(t)^{2} d t=\int_{C}(b q(t))^{2} d t+\int_{B} a^{2} d t=b^{2} \cdot 1+a^{2} m(B)=$ $b^{2}+\left(1-b^{2}\right)=1$ so that $\hat{q} \in A_{0}$.

Note $\int_{0}^{1}(q(t)-\hat{q}(t))^{2} d t=\int_{C}(q(t)-b q(t))^{2} d t+\int_{B} a^{2} d t=(1-b)^{2}+a^{2} m(B)=$ $(1-b)^{2}+\left(1-b^{2}\right)=1-2 b+b^{2}+1-b^{2}=2-2 b=2(1-b)<2 \epsilon / 2=\epsilon$ so that $q$ is in the closure of $A_{0}$ in $L^{2}[0,1]$ and this is true for every $q$ in $A \backslash A_{0}$. Finally, if $q \notin A$, we show $q$ is not in the closure of $A_{0}$ in $L^{2}[0,1]$. If $\|q\|_{2} \neq 1$, then clearly $q$ is not in the closure. Thus, assume $\|q\|_{2}=1$. Since $q \notin A$, then a measurable subset $B$ of $[0,1]$ exists, $m(B)>0$, on which $q<0$. Thus, $\int_{B} q(t)^{2}>0$ (i of Proposition 2.34) so that $\int_{B}(q(t)-\hat{q}(t))^{2} d t>\int_{B} q(t)^{2} d t$ for any $\hat{q} \in A_{0}$. Thus, $q$ can not be in the closure of $A_{0}$ in $L^{2}[0,1]$ and $A$ must then be the closure of $A_{0}$ in $L^{2}[0,1]$.

Corollary 4.4 (SRSF's of functions in $\Gamma_{0}$ and $\Gamma$; orbit of the constant function equal to 1 [10]): $Q_{0}=\left\{\bar{q}: \bar{q}=\sqrt{\dot{\gamma}}\right.$ a.e. on $[0,1]$, some $\left.\gamma \in \Gamma_{0}\right\}$ has closure in $L^{2}[0,1]$ equal to $Q=\{\bar{q}: \bar{q}=\sqrt{\dot{\gamma}}$ a.e. on $[0,1]$, some $\gamma \in \Gamma\}$. In addition, with $A_{0}=\left\{q \in L^{2}[0,1]:\|q\|_{2}=1, q>0\right.$ a.e. on $\left.[0,1]\right\}$, $A=\left\{q \in L^{2}[0,1]:\|q\|_{2}=1, q \geq 0\right.$ a.e. on $\left.[0,1]\right\}$, and $q_{0}$ the constant function equal to 1 on $[0,1]$, then $\left[q_{0}\right]_{\Gamma_{0}}=Q_{0}=A_{0}$ and $\operatorname{cl}\left(\left[q_{0}\right]_{\Gamma_{0}}\right)=Q=A$. 
Proof: If $\gamma \in \Gamma_{0}$ and $\bar{q}=\sqrt{\dot{\gamma}}$ a.e. on $[0,1]$, then $\int_{0}^{1} \bar{q}(t)^{2} d t=\int_{0}^{1} \dot{\gamma}(t) d t=$ $\gamma(1)-\gamma(0)=1$. Also $\bar{q}>0$ a.e. on $[0,1]$ since $\gamma \in \Gamma_{0}$ so that $\bar{q} \in A_{0}$. Thus, $Q_{0} \subseteq A_{0}$. On the other hand, if $q \in A_{0}$, then $q>0$ a.e. on $[0,1]$, $q \in L^{2}[0,1],\|q\|_{2}=1$. By Proposition 4.3, $\gamma$ defined for each $t \in[0,1]$ by $\gamma(t)=\int_{0}^{t} q(s)|q(s)| d s=\int_{0}^{t} q(s)^{2} d s$ is absolutely continuous on $[0,1]$ with $q$ equal to the SRSF of $\gamma$ a.e. on $[0,1]$. Clearly $\gamma(0)=0, \gamma(1)=\|q\|_{2}=1$, $\dot{\gamma}=q^{2}$ a.e. on $[0,1]$, thus $\gamma \in \Gamma_{0}$ and $\sqrt{\dot{\gamma}}=q$ a.e. on $[0,1]$ so that $q \in Q_{0}$. Thus $Q_{0}=A_{0}$. Similarly, $Q=A$ so that the closure of $Q_{0}$ in $L^{2}[0,1]$ is $Q$ by Proposition 4.12. Finally, $\left[q_{0}\right]_{\Gamma_{0}}=\left\{\bar{q}: \bar{q}=\left(q_{0}, \gamma\right)=\left(q_{0} \circ \gamma\right) \sqrt{\dot{\gamma}}=\right.$ $\sqrt{\dot{\gamma}}$ a.e. on $[0,1]$, some $\left.\gamma \in \Gamma_{0}\right\}=Q_{0}=A_{0}$, and since, as just proved, the closure of $Q_{0}$ in $L^{2}[0,1]$ is $Q$, then $\operatorname{cl}\left(\left[q_{0}\right]_{\Gamma_{0}}\right)=Q=A$.

Corollary 4.5 ([10]): With $q_{0}$ the constant function equal to 1 on $[0,1]$, given $q_{1}, q_{2} \in L^{2}[0,1],\left\|q_{1}\right\|_{2}=\left\|q_{2}\right\|_{2}=1$, if either (i) $q_{1} \geq 0$ a.e. on $[0,1]$ and $q_{2} \geq 0$ a.e. on [0,1], or (ii) $q_{1} \leq 0$ a.e. on $[0,1]$ and $q_{2} \leq 0$ a.e. on $[0,1]$, then in the case of (i) it must be that $c l\left(\left[q_{1}\right]_{\Gamma_{0}}\right)=\operatorname{cl}\left(\left[q_{2}\right]_{\Gamma_{0}}\right)=c l\left(\left[q_{0}\right]_{\Gamma_{0}}\right)$, and in the case of (ii) it must be that $c l\left(\left[q_{1}\right]_{\Gamma_{0}}\right)=\operatorname{cl}\left(\left[q_{2}\right]_{\Gamma_{0}}\right)=\operatorname{cl}\left(\left[-q_{0}\right]_{\Gamma_{0}}\right)$. In both cases a sequence $\left\{\gamma_{n}\right\}$ exists in $\Gamma_{0}$ with $\left(q_{1}, \gamma_{n}\right) \rightarrow q_{2}$ in $L^{2}[0,1]$.

Proof: With $A=\left\{q \in L^{2}[0,1]:\|q\|_{2}=1, q \geq 0\right.$ a.e. on $\left.[0,1]\right\}$, if (i) is true, then $q_{1}, q_{2} \in A=\operatorname{cl}\left(\left[q_{0}\right]_{\Gamma_{0}}\right)$ (Corollary 4.4) so that by Proposition 4.10, $\operatorname{cl}\left(\left[q_{1}\right]_{\Gamma_{0}}\right)=\operatorname{cl}\left(\left[q_{2}\right]_{\Gamma_{0}}\right)=\operatorname{cl}\left(\left[q_{0}\right]_{\Gamma_{0}}\right)$. On the other hand, if (ii) is true, then using similar arguments as above with $-q_{0}=-1$ taking the place of $q_{0}$, it then follows that $\operatorname{cl}\left(\left[q_{1}\right]_{\Gamma_{0}}\right)=\operatorname{cl}\left(\left[q_{2}\right]_{\Gamma_{0}}\right)=\operatorname{cl}\left(\left[-q_{0}\right]_{\Gamma_{0}}\right)$. In both cases $c l\left(\left[q_{1}\right]_{\Gamma_{0}}\right)=c l\left(\left[q_{2}\right]_{\Gamma_{0}}\right)$ implies the existence of $\left\{\gamma_{n}\right\}$.

Corollary 4.6 ([10]): Given $q_{1}, q_{2} \in L^{2}[0,1]$, and a sequence of numbers $t_{0}=0<t_{1}<\ldots<t_{n}=1$, such that for each $i, i=1, \ldots, n$, $\int_{t_{i-1}}^{t_{i}} q_{1}(t)^{2} d t=\int_{t_{i-1}}^{t_{i}} q_{2}(t)^{2} d t$, and either $q_{1} \geq 0$ and $q_{2} \geq 0$ a.e. on $\left[t_{i-1}, t_{i}\right]$, or $q_{1} \leq 0$ and $q_{2} \leq 0$ a.e. on $\left[t_{i-1}, t_{i}\right]$, then $\operatorname{cl}\left(\left[q_{1}\right]_{\Gamma_{0}}\right)=\operatorname{cl}\left(\left[q_{2}\right]_{\Gamma_{0}}\right)$.

Proof: Given $i, 1 \leq i \leq n$, then a sequence $\left\{\lambda_{n}^{i}\right\}$ exists of absolutely continuous functions, $\lambda_{n}^{i}:\left[t_{i-1}, t_{i}\right] \rightarrow\left[t_{i-1}, t_{i}\right], \dot{\lambda}_{n}^{i}>0$ a.e. on $\left[t_{i-1}, t_{i}\right]$, $\lambda_{n}^{i}\left(t_{i-1}\right)=t_{i-1}, \lambda_{n}^{i}\left(t_{i}\right)=t_{i}$ for each $n$ such that $\left(q_{1}, \lambda_{n}^{i}\right) \rightarrow q_{2}$ in $L^{2}\left[t_{i-1}, t_{i}\right]$. Here $\left(q_{1}, \lambda_{n}^{i}\right)$ is understood to be $\left(q_{1} \circ \lambda_{n}^{i}\right) \sqrt{\dot{\lambda}_{n}^{i}}$ and $L^{2}\left[t_{i-1}, t_{i}\right]$ the set of squareintegrable functions over $\left[t_{i-1}, t_{i}\right]$. Proof of the existence of $\left\{\lambda_{n}^{i}\right\}$ along the 
lines of that of Corollary 4.5 with $\left[t_{i-1}, t_{i}\right]$ taking the place of $[0,1]$ and the value of $\int_{t_{i-1}}^{t_{i}} q_{1}(t)^{2} d t=\int_{t_{i-1}}^{t_{i}} q_{2}(t)^{2} d t$ not necessarily equal to 1 .

Finally, define a sequence of functions $\left\{\gamma_{n}\right\}, \gamma_{n}:[0,1] \rightarrow[0,1]$, by setting $\gamma_{n}(t)=\lambda_{n}^{i}(t)$ if $t \in\left[t_{i-1}, t_{i}\right]$ for each $n$. It follows $\gamma_{n}$ is absolutely continuous, $\gamma_{n}(0)=0, \gamma_{n}(1)=1, \dot{\gamma}_{n}>0$ a.e. on $[0,1]$ for each $n$. Thus $\left\{\gamma_{n}\right\} \subset \Gamma_{0}$ and since $\left(q_{1}, \lambda_{n}^{i}\right) \rightarrow q_{2}$ in $L^{2}\left[t_{i-1}, t_{i}\right]$ for each $i$, then $\left(q_{1}, \gamma_{n}\right) \rightarrow q_{2}$ in $L^{2}[0,1]$. Thus, $q_{2} \in \operatorname{cl}\left(\left[q_{1}\right]_{\Gamma_{0}}\right)$ and by Proposition 4.10, then $\operatorname{cl}\left(\left[q_{1}\right]_{\Gamma_{0}}\right)=\operatorname{cl}\left(\left[q_{2}\right]_{\Gamma_{0}}\right)$.

Corollary 4.7 ([10]): Given $q_{1}, q_{2} \in L^{2}[0,1]$, and two sequences of numbers $t_{0}=0<t_{1}<\ldots<t_{n}=1, t_{0}^{\prime}=0<t_{1}^{\prime}<\ldots<t_{n}^{\prime}=1$, such that for each $i$, $i=1, \ldots, n, \int_{t_{i-1}}^{t_{i}} q_{1}(t)^{2} d t=\int_{t_{i-1}^{\prime}}^{t_{i}^{\prime}} q_{2}(t)^{2} d t$, and either $q_{1} \geq 0$ a.e. on $\left[t_{i-1}, t_{i}\right]$ and $q_{2} \geq 0$ a.e. on $\left[t_{i-1}^{\prime}, t_{i}^{\prime}\right]$, or $q_{1} \leq 0$ a.e. on $\left[t_{i-1}, t_{i}\right]$ and $q_{2} \leq 0$ a.e. on $\left[t_{i-1}^{\prime}, t_{i}^{\prime}\right]$, then $\operatorname{cl}\left(\left[q_{1}\right]_{\Gamma_{0}}\right)=\operatorname{cl}\left(\left[q_{2}\right]_{\Gamma_{0}}\right)$.

Proof: Let $\gamma$ be the piecewise linear element of $\Gamma_{0}$ for which $\gamma\left(t_{i}^{\prime}\right)=t_{i}$, $i=0, \ldots, n$, and let $w=\left(q_{1}, \gamma\right)$. It then follows by Corollary 3.14 (change of variable) that for each $i, i=1, \ldots, n, \int_{t_{i-1}^{\prime}}^{t_{i}^{\prime}} w(t)^{2} d t=\int_{t_{i-1}^{\prime}}^{t_{i}^{\prime}}\left(q_{1}(\gamma(t))\right)^{2} \dot{\gamma}(t) d t=$ $\int_{t_{i-1}}^{t_{i}} q_{1}(s)^{2} d s=\int_{t_{i-1}^{\prime}}^{t_{i}^{\prime}} q_{2}(t)^{2} d t$, and since $w \geq 0$ a.e. on $\left[t_{i-1}^{\prime}, t_{i}^{\prime}\right]$ if $q_{1} \geq 0$ a.e. on $\left[t_{i-1}, t_{i}\right]$, and $w \leq 0$ a.e. on $\left[t_{i-1}^{\prime}, t_{i}^{\prime}\right]$ if $q_{1} \leq 0$ a.e. on $\left[t_{i-1}, t_{i}\right]$, then $w$ and $q_{2}$ satisfy the hypothesis of Corollary 4.6 for the sequence $\left\{t_{n}^{\prime}\right\}$ so that $c l\left([w]_{\Gamma_{0}}\right)=\operatorname{cl}\left(\left[q_{2}\right]_{\Gamma_{0}}\right)$. Since $w=\left(q_{1}, \gamma\right), \gamma \in \Gamma_{0}$, then $w \in\left[q_{1}\right]_{\Gamma_{0}}$ so that by Observation 4.12, $[w]_{\Gamma_{0}}=\left[q_{1}\right]_{\Gamma_{0}}$ and therefore $\operatorname{cl}\left(\left[q_{1}\right]_{\Gamma_{0}}\right)=\operatorname{cl}\left(\left[q_{2}\right]_{\Gamma_{0}}\right)$.

Proposition 4.13 ([10]): Given $q \in L^{2}[0,1]$, then $[q]_{\Gamma} \subseteq \operatorname{cl}\left([q]_{\Gamma_{0}}\right)$.

Proof: The proposition is first proved for step functions on [0,1]. Accordingly, we assume $q$ is a step function and $\gamma \in \Gamma$.

Let $t_{0}=0<t_{1}<\ldots<t_{n}=1$ be the set of numbers that define the partition associated with $q$ as a step function. For each $i, i=0, \ldots, n$, let $t_{i}^{\prime} \in[0,1]$ be such that $\gamma\left(t_{i}^{\prime}\right)=t_{i}$ with $t_{0}^{\prime}=0$ and $t_{n}^{\prime}=1$. Note $t_{0}^{\prime}=0<t_{1}^{\prime}<\ldots<t_{n}^{\prime}=1$, as $\gamma$ is a nondecreasing function from $[0,1]$ onto $[0,1]$.

Let $w=(q, \gamma)$. It then follows by Corollary 3.14 (change of variable) that for each $i, i=1, \ldots, n, \int_{t_{i-1}^{\prime}}^{t_{i}^{\prime}} w(t)^{2} d t=\int_{t_{i-1}^{\prime}}^{t_{i}^{\prime}}(q(\gamma(t)))^{2} \dot{\gamma}(t) d t=\int_{t_{i-1}}^{t_{i}} q(s)^{2} d s$, and either $q \geq 0$ a.e. on $\left[t_{i-1}, t_{i}\right]$ and $w \geq 0$ a.e. on $\left[t_{i-1}^{\prime}, t_{i}^{\prime}\right]$, or $q \leq 0$ a.e. on $\left[t_{i-1}, t_{i}\right]$ and $w \leq 0$ a.e. on $\left[t_{i-1}^{\prime}, t_{i}^{\prime}\right]$. Thus, by Corollary 4.7, 
$\operatorname{cl}\left([q]_{\Gamma_{0}}\right)=\operatorname{cl}\left([w]_{\Gamma_{0}}\right)$ so that, in particular, $w=(q, \gamma) \in \operatorname{cl}\left([q]_{\Gamma_{0}}\right)$ and therefore, since $\gamma$ is arbitrary in $\Gamma$, then $[q]_{\Gamma} \subseteq \operatorname{cl}\left([q]_{\Gamma_{0}}\right)$.

Finally, we assume $q$ is any function in $L^{2}[0,1]$ and $\gamma \in \Gamma$. Given $\epsilon>0$, by Proposition 2.44 (density of step functions in $L^{p}$ ), there is a step function $v$ on $[0,1]$ such that $\|q-v\|_{2}<\epsilon / 3$. As just proved above, $(v, \gamma) \in \operatorname{cl}\left([v]_{\Gamma_{0}}\right)$ so that for some $\bar{\gamma} \in \Gamma_{0}$ it must be that $\|(v, \gamma)-(v, \bar{\gamma})\|_{2}<\epsilon / 3$. Thus, by Corollary 4.1 and Observation 4.8 (action of $\Gamma_{0}$ and $\Gamma$ is distance preserving)

$$
\begin{aligned}
\|(q, \gamma)-(q, \bar{\gamma})\|_{2} & =\|(q, \gamma)-(v, \gamma)\|_{2}+\|(v, \gamma)-(v, \bar{\gamma})\|_{2} \\
& +\|(v, \bar{\gamma})-(q, \bar{\gamma})\|_{2} \\
& =\|q-v\|_{2}+\|(v, \gamma)-(v, \bar{\gamma})\|_{2}+\|v-q\|_{2} \\
& <\epsilon / 3+\epsilon / 3+\epsilon / 3=\epsilon
\end{aligned}
$$

so that $(q, \gamma) \in \operatorname{cl}\left([q]_{\Gamma_{0}}\right)$ and therefore, since $\gamma$ is arbitrary in $\Gamma$, then $[q]_{\Gamma} \subseteq$ $\operatorname{cl}\left([q]_{\Gamma_{0}}\right)$.

Proposition 4.14 (Constant-speed parametrization of an absolutely continuous function [24]): Given $f \in A C[0,1]$, then there exist $h \in$ $A C[0,1], \gamma \in \Gamma$, such that $\left|h^{\prime}\right|=L=\int_{0}^{1}\left|f^{\prime}(t)\right| d t$ (the length of $f$ ) a.e. on $[0,1]$ and $f=h \circ \gamma$ on $[0,1]$.

Proof: Given $f \in A C[0,1]$, let $L=\int_{0}^{1}\left|f^{\prime}(t)\right| d t$. If $L=0$ then $f$ is constant on $[0,1]$ (i of Proposition 2.34, Proposition 3.8). Otherwise, define $\gamma:[0,1] \rightarrow$ $[0,1]$ by $\gamma(t)=(1 / L) \int_{0}^{t}\left|f^{\prime}(s)\right| d s$ for each $t \in[0,1]$. Accordingly, $\gamma(0)=0$, $\gamma(1)=(1), \gamma \in A C[0,1]$ by Proposition 3.11, and $\dot{\gamma}(t)=(1 / L)\left|f^{\prime}(t)\right|$ a.e. on $[0,1]$ by Proposition 3.7 so that $\dot{\gamma} \geq 0$ a.e. on $[0,1]$. Thus $\gamma \in \Gamma$.

Given $s \in[0,1]$, then for some $t \in[0,1]$ it must be that $\gamma(t)=s$. Define $h:[0,1] \rightarrow \mathbf{R}$ by $h(s)=f(t)$. The function $h$ is well defined for if $s=\gamma\left(t_{1}\right)=$ $\gamma\left(t_{2}\right), t_{1}<t_{2} \in[0,1]$, then $0=\int_{0}^{t_{2}}\left|f^{\prime}(x)\right| d x-\int_{0}^{t_{1}}\left|f^{\prime}(x)\right| d x=\int_{t_{1}}^{t_{2}}\left|f^{\prime}(x)\right| d x$. Thus, by i of Proposition $2.34, f^{\prime}=0$ a.e. on $\left[t_{1}, t_{2}\right]$ so that by Proposition 3.8 $f$ is constant on $\left[t_{1}, t_{2}\right]$ and, in particular, $f\left(t_{1}\right)=f\left(t_{2}\right)$.

Clearly $h(\gamma(t))=f(t)$ for each $t \in[0,1]$. Note for $s_{1}, s_{2} \in[0,1], s_{1}<s_{2}$, then $s_{1}=\gamma\left(t_{1}\right), s_{2}=\gamma\left(t_{2}\right), t_{1}, t_{2} \in[0,1], t_{1}<t_{2}$, and

$$
\left|h\left(s_{2}\right)-h\left(s_{1}\right)\right|=\left|f\left(t_{2}\right)-f\left(t_{1}\right)\right|=\left|\int_{t_{1}}^{t_{2}} f^{\prime}(x) d x\right| \leq \int_{t_{1}}^{t_{2}}\left|f^{\prime}(x)\right| d x=L \cdot\left(s_{2}-s_{1}\right) .
$$

From this inequality it follows clearly that $h \in A C[0,1]$ (Definition 3.5). Accordingly, $h$ is differentiable a.e. on $[0,1]$ and $\left|h^{\prime}\right| \leq L$ a.e. on $[0,1]$ also 
from the inequality. Note that by Corollary 3.14 (change of variable) and Corollary 3.13 (chain rule), then

$$
\int_{0}^{1}\left|h^{\prime}(s)\right| d s=\int_{0}^{1}\left|h^{\prime}(\gamma(t))\right| \dot{\gamma}(t) d t=\int_{0}^{1}\left|f^{\prime}(t)\right| d t=L .
$$

By i of Proposition 2.34, then $\left|h^{\prime}\right|=L$ a.e. on $[0,1]$.

Corollary 4.8: Given $q \in L^{2}[0,1]$, then there exist $w \in L^{2}[0,1], \gamma \in \Gamma$, such that $|w|=\sqrt{L}$ a.e. on $[0,1]$ and $q=(w, \gamma)$ a.e. on $[0,1]$, where $L=\int_{0}^{1}\left|f^{\prime}(t)\right| d t$ (the length of $f$ ), $f \in A C[0,1]$, the SRSF of $f$ equal to $q$ a.e. on $[0,1]$. In particular, if $\|q\|_{2}=1$ so that $L=1$, then $|w|=1$ a.e. on $[0,1]$.

Definition 4.8: A function $q \in L^{2}[0,1]$, is said to be in standard form if for measurable subsets $A, B$ of $[0,1]$, with $A \cap B=\emptyset, A \cup B=[0,1]$, then

$$
q(t)= \begin{cases}1 & \text { for } t \in A \\ -1 & \text { for } t \in B\end{cases}
$$

Clearly, if $q$ is in standard form, then $\|q\|_{2}=1$.

Let $S F[0,1]=\left\{q: q \in L^{2}[0,1], q\right.$ in standard form $\}$.

Proposition 4.15 ([10]): Given $q, w \in S F[0,1]$, if $q \neq w$ in $L^{2}$, i.e., if $m(\{t$ : $t \in[0,1], q(t) \neq w(t)\})>0$, then $w \notin c l\left([q]_{\Gamma_{0}}\right)$. Thus $c l\left([w]_{\Gamma_{0}}\right) \cap c l\left([q]_{\Gamma_{0}}\right)=\emptyset$. Proof in [10] using Corollary 3.16 (Change of variable for Lebesgue integral over a measurable set) and Observation 2.25 (Schwarz's inequality over a measurable set).

Corollary 4.9: (Uniqueness of constant-speed parametrization): Given $\tilde{q} \in L^{2}[0,1],\|\tilde{q}\|_{2}=1$, if for $\gamma, \tilde{\gamma} \in \Gamma$, and $q, w \in S F[0,1], \tilde{q}=(q, \gamma)$ and $\tilde{q}=(w, \tilde{\gamma})$, then $q=w$ a.e. on $[0,1]$.

Proof: By Proposition 4.13, $[q]_{\Gamma} \subseteq \operatorname{cl}\left([q]_{\Gamma_{0}}\right)$ and $[w]_{\Gamma} \subseteq \operatorname{cl}\left([w]_{\Gamma_{0}}\right)$. Thus, $\tilde{q} \in \operatorname{cl}\left([q]_{\Gamma_{0}}\right) \cap \operatorname{cl}\left([w]_{\Gamma_{0}}\right)$ so that by Proposition $4.15, q=w$ a.e. on $[0,1]$.

Proposition 4.16 ([10]): Given $w \in S F[0,1]$, then $c l\left([w]_{\Gamma_{0}}\right)=[w]_{\Gamma}$.

Proof: From Proposition 4.13, we know $[w]_{\Gamma} \subseteq \operatorname{cl}\left([w]_{\Gamma_{0}}\right)$. Thus, it suffices 
to show $c l\left([w]_{\Gamma_{0}}\right) \subseteq[w]_{\Gamma}$. For this purpose, let $\tilde{q}$ be in $\operatorname{cl}\left([w]_{\Gamma_{0}}\right)$. Clearly $\|\tilde{q}\|_{2}=1$, and by Corollary 4.8, for some $q \in S F[0,1]$, and some $\gamma \in \Gamma$, it must be that $\tilde{q}=(q, \gamma)$ a.e. on $[0,1]$. By Proposition 4.13, $[q]_{\Gamma} \subseteq \operatorname{cl}\left([q]_{\Gamma_{0}}\right)$. Thus, $\tilde{q} \in \operatorname{cl}\left([q]_{\Gamma_{0}}\right) \cap \operatorname{cl}\left([w]_{\Gamma_{0}}\right)$ so that by Proposition $4.15, q=w$ a.e. on $[0,1]$, and therefore $\tilde{q}$ is in $[w]_{\Gamma}$. Thus $\operatorname{cl}\left([w]_{\Gamma_{0}}\right) \subseteq[w]_{\Gamma}$.

Corollary 4.10 ([10]): Given $q \in L^{2}[0,1]$, if $q \neq 0$ a.e. on $[0,1]$, then $\operatorname{cl}\left([q]_{\Gamma_{0}}\right)=[q]_{\Gamma}$.

Proof: If $\|q\|_{2}=1$, then by Corollary 4.8, for some $w \in S F[0,1]$, and some $\gamma \in \Gamma$, it must be that $q=(w, \gamma)$ a.e. on $[0,1]$. Since $q \neq 0$ a.e on $[0,1]$, then $(w, \gamma)=(w \circ \gamma) \sqrt{\dot{\gamma}} \neq 0$ a.e. on $[0,1]$, and therefore $\dot{\gamma} \neq 0$ a.e. on $[0,1]$. Thus, $\gamma \in \Gamma_{0}$ so that $[q]_{\Gamma_{0}}=[w]_{\Gamma_{0}}$ and $\left(q, \gamma^{-1}\right)=\left((w, \gamma), \gamma^{-1}\right)=w$ a.e. on $[0,1]$ (Proposition 4.5). Accordingly, $\operatorname{cl}\left([q]_{\Gamma_{0}}\right)=\operatorname{cl}\left([w]_{\Gamma_{0}}\right)=[w]_{\Gamma}=$ $\left[\left(q, \gamma^{-1}\right)\right]_{\Gamma}$ (Proposition 4.16). Given $\tilde{q} \in\left[\left(q, \gamma^{-1}\right)\right]_{\Gamma}$, then for some $\tilde{\gamma} \in \Gamma$, $\tilde{q}=\left(\left(q, \gamma^{-1}\right), \tilde{\gamma}\right)=\left(q, \gamma^{-1} \circ \tilde{\gamma}\right)$ a.e. on $[0,1]$ (Proposition 4.5), and since $\gamma^{-1} \circ \tilde{\gamma} \in \Gamma$ (Observation 4.4), then $\tilde{q} \in[q]_{\Gamma}$. On the other hand, given $\tilde{q} \in[q]_{\Gamma}$, then for some $\tilde{\gamma} \in \Gamma, \tilde{q}=(q, \tilde{\gamma})=\left(q, \gamma^{-1} \circ \gamma \circ \tilde{\gamma}\right)=\left(\left(q, \gamma^{-1}\right), \gamma \circ \tilde{\gamma}\right)$ a.e. on $[0,1]$ (Proposition 4.5), and since $\gamma \circ \tilde{\gamma} \in \Gamma$ (Observation 4.4), then $\tilde{q} \in\left[\left(q, \gamma^{-1}\right)\right]_{\Gamma}$. Thus $\left[\left(q, \gamma^{-1}\right)\right]_{\Gamma}=[q]_{\Gamma}$ and therefore $\operatorname{cl}\left([q]_{\Gamma_{0}}\right)=[q]_{\Gamma}$. If $\|q\|_{2} \neq 1$, then clearly $\|q\|_{2} \neq 0, q /\|q\|_{2} \neq 0$ a.e. on $[0,1]$, and as just proved $c l\left(\left[q /\|q\|_{2}\right]_{\Gamma_{0}}\right)=\left[q /\|q\|_{2}\right]_{\Gamma}$. Given $\tilde{q} \in \operatorname{cl}\left([q]_{\Gamma_{0}}\right)$, then for a sequence $\left\{\gamma_{n}\right\} \subset \Gamma_{0},\left(q, \gamma_{n}\right) \rightarrow \tilde{q}$ in $L^{2}$. Thus $\left(q /\|q\|_{2}, \gamma_{n}\right) \rightarrow \tilde{q} /\|q\|_{2}$ in $L^{2}$ implying $\tilde{q} /\|q\|_{2}=\left(q /\|q\|_{2}, \gamma\right)$ for some $\gamma \in \Gamma$, and therefore $\tilde{q}=(q, \gamma)$ so that $\tilde{q} \in[q]_{\Gamma}$. On the other hand, given $\tilde{q} \in[q]_{\Gamma}$, then for some $\gamma \in \Gamma$, $\tilde{q}=(q, \gamma)$. Thus $\tilde{q} /\|q\|_{2}=\left(q /\|q\|_{2}, \gamma\right)$ implying for a sequence $\left\{\gamma_{n}\right\} \subset \Gamma_{0}$, $\left(q /\|q\|_{2}, \gamma_{n}\right) \rightarrow \tilde{q} /\|q\|_{2}$ in $L^{2}$, and therefore $\left(q, \gamma_{n}\right) \rightarrow \tilde{q}$ in $L^{2}$ so that $\tilde{q} \in \operatorname{cl}\left([q]_{\Gamma_{0}}\right)$. Thus $c l\left([q]_{\Gamma_{0}}\right)=[q]_{\Gamma}$.

Observation 4.17: As noted in [10], given $f_{1}, f_{2} \in A C[0,1]$, and their SRSF's $q_{1}, q_{2} \in L^{2}[0,1]$, respectively, if $\tilde{q}_{1} \in \operatorname{cl}\left(\left[q_{1}\right]_{\Gamma_{0}}\right), \tilde{q}_{2} \in \operatorname{cl}\left(\left[q_{2}\right]_{\Gamma_{0}}\right)$ exist such that $d\left(c l\left(\left[q_{1}\right]_{\Gamma_{0}}\right), \operatorname{cl}\left(\left[q_{2}\right]_{\Gamma_{0}}\right)\right)=\left\|\tilde{q}_{1}-\tilde{q}_{2}\right\|_{2}$, assuming without any loss of generality that $q_{1} \neq 0$ a.e. on $[0,1], q_{2} \neq 0$ a.e. on $[0,1]$ (Corollary 4.8), then by Corollary 4.10 above there exist $\gamma_{1}, \gamma_{2} \in \Gamma$, such that $\tilde{q}_{1}=\left(q_{1}, \gamma_{1}\right)$ and $\tilde{q}_{2}=\left(q_{2}, \gamma_{2}\right)$. The pair $\gamma_{1}, \gamma_{2}$ is called an optimal matching for $f_{1}, f_{2}$. In particular, it is proved in [10] that if at least one of $c l\left(\left[q_{1}\right]_{\Gamma_{0}}\right), \operatorname{cl}\left(\left[q_{2}\right]_{\Gamma_{0}}\right)$ contains the SRSF of a piecewise linear function, then $\tilde{q}_{1}, \tilde{q}_{2}$ exist as above 
and therefore there is an optimal matching for $f_{1}, f_{2}$. This is actually proved in [10] for absolutely continuous functions $f_{1}, f_{2}$ with range $\mathbf{R}^{n}$.

\section{Summary}

In order to understand the theory of functional data and shape analysis as presented in Srivastava and Klassen's textbook "Functional and Shape Data Analysis" [23], it is important to understand the basics of Lebesgue integration and absolute continuity, and the connections between them. In this paper of the survey type, we have tried to provide a way to do exactly that. We have reviewed fundamental concepts and results about Lebesgue integration and absolute continuity, some results connecting the two notions, most of the material borrowed from Royden's "Real Analysis" [16] and Rudin's "Principles of Mathematical Analysis" [18]. Additional important material was obtained from Saks' [20], and Serrin and Varberg's [22] seminal papers. In addition, we have presented fundamental concepts and results about functional data and shape analysis in 1-dimensional space, in the process shedding light on its dependence on Lebesgue integration and absolute continuity, and the connections between them, most of the material borrowed from Srivastava and Klassen's aforementioned textbook. Additional material presented at the end of the paper was obtained from Lahiri, Robinson and Klassen's outstanding manuscript [10].

\section{Acknowledgements}

I am most grateful to Professor James F. Lawrence of George Mason University and the National Institute of Standards and Technology for the many insightful conversations on the subjects of Lebesgue integration and absolute continuity, and to Professor Eric Klassen of Florida State University for his generosity in always providing answers to my questions about his remarkable work on shape analysis.

\section{References}

[1] Apostol, T. M.: Mathematical Analysis, 2nd edition. Reading, Massachusetts: Addison-Wesley Publishing Company. (1974) 
[2] Bernal, J., Dogan, G., Hagwood, C. R.: Fast Dynamic Programming for Elastic Registration of Curves. Proceedings of DIFF-CVML workshop, CVPR 2016, Las Vegas, Nevada. (2016)

[3] Bruckner, A. M., Bruckner, J. B., Thomson, B. S.: Real Analysis, 1st edition. Upper Saddle River, New Jersey: Prentice-Hall. (1997)

[4] Burk, F.: Lebesgue Measure and Integration. An Introduction, 1st edition. New York: John Wiley \& Sons. (1998)

[5] Cabada, A., Pouso, R. L.: On First Order Discontinuous Scalar Differential Equations. Nonlinear Studies 2 (1999) 161-170.

[6] do Carmo, M. P.: Differential Geometry of Curves and Surfaces. Upper Saddle River, New Jersey: Prentice-Hall. (1976)

[7] do Carmo, M. P.: Riemannian Geometry. Boston: Birkhäuser. (1992)

[8] Halmos, P. R.: Measure Theory. New York: Springer-Verlag. (1974)

[9] Klassen, E.: Private communication. (2018)

[10] Lahiri, S., Robinson, D., Klassen, E.: Precise Matching of PL curves in $\mathbf{R}^{n}$ in the Square Root Velocity Framework. Geometry, Imaging and Computing 2(3) (2015) 133-186.

[11] Lee, J. M.: Introduction to Smooth Manifolds. Graduate Texts in Mathematics, Vol. 218. New York: Springer-Verlag. (2003)

[12] Lee, J. M.: Riemannian Manifolds. An Introduction to Curvature. Graduate Texts in Mathematics, Vol. 176. New York: Springer-Verlag. (1997)

[13] Pugh, C. C.: Real Mathematical Analysis, 2nd edition. New York: Springer. (2015)

[14] Rana, I. K.: An Introduction to Measure and Integration, 2nd edition. Graduate Studies in Mathematics, Vol. 45. Providence, Rhode Island: American Mathematical Society. (2002)

[15] Richardson, L. F.: Advanced Calculus. An introduction to Linear Analysis, 1st edition. Hoboken, New Jersey: John Wiley \& Sons. (2008) 
[16] Royden, H. L.: Real Analysis, 2nd edition. New York: Macmillan. (1968)

[17] Royden, H. L., Fitzpatrick P. M.: Real Analysis, 4th edition. Upper Saddle River, New Jersey: Pearson Education. (2010)

[18] Rudin, W.: Principles of Mathematical Analysis, 2nd edition. New York: McGraw-Hill. (1964)

[19] Rudin, W.: Real and Complex Analysis, 3rd edition. New York: McGraw-Hill. (1987)

[20] Saks, S.: Theory of the Integral. Monografie Matematyczne Tom VII, 2nd revised edition. New York: Hafner Publishing Co. (1937)

[21] Schmeding, A.: Manifolds of Absolutely Continuous Curves and the Square Root Velocity Framework. arXiv preprint arXiv:1612.02604 (2016)

[22] Serrin, J., Varberg, D. E.: A General Chain Rule for Derivatives and the Change of Variables Formula for the Lebesgue Integral. The American Mathematical Monthly 76(5) (1969) 514-520.

[23] Srivastava, A., Klassen, E. P.: Functional and Shape Data Analysis. New York: Springer. (2016)

[24] Stein, E. M., Shakarchi, R.: Real Analysis: Measure Theory, Integration, and Hilbert Spaces. Princeton, New Jersey: Princeton University Press. (2005)

[25] Wade, W. R.: An Introduction to Analysis, 3rd edition. Upper Saddle River, New Jersey: Pearson Prentice Hall. (2004)

[26] Yeh, J. Real Analysis. Theory of Measure and Integration, 3rd edition. Hackensack, New Jersey: World Scientific Publishing Co. (2014)

\section{Index of Terms}

Each entry crossreferenced to one of the following: Dx.y (Definition x.y), Px.y (Proposition x.y), Cx.y (Corollary x.y), Ox.y (Observation x.y).

Given x.y, $y$ is then the number of the Definition, Proposition, Corollary or Observation in Section $\mathrm{x}$ in which the entry can be found. For example, the entry "Borel" is associated in the index with D2.3 which means it can be 
found in Definition 3 of Section 2.

Absolute continuity of the indefinite integral, P3.11

Absolute continuity of the Lebesgue integral, P2.38

absolutely continuous, D3.5

Absolutely continuous $f$ implies $f$ is of bounded variation, P3.10

Absolutely continuous $f$ is constant if $f^{\prime}$ is zero a.e., P3.8

Absolutely continuous $f$ maps measurable sets to measurable sets, P3.21

Absolutely continuous $f$ maps zero-measure sets to zero-measure sets, P3.21

$A C[0,1], \mathrm{O} 4.1$

$A C^{0}[0,1], \mathrm{D} 4.4$

action of $\Gamma$ on $L^{2}[0,1]$, D4.3

Action of $\Gamma$ on $L^{2}[0,1]$ is by semi-isometries, P4.6

Action of $\Gamma$ on $L^{2}[0,1]$ is distance and norm preserving, $\mathrm{O} 4.8$

action of $\Gamma_{0}$ on $A C^{0}[0,1], \mathrm{D} 4.5$

Action of $\Gamma_{0}$ on $A C^{0}[0,1]$ with Fisher-Rao metric is by isometries, $\mathrm{P} 4.8$

action of $\Gamma_{0}$ on $L^{2}[0,1], \mathrm{D} 4.3$

Action of $\Gamma_{0}$ on $L^{2}[0,1]$ is by isometries, $\mathrm{P} 4.6$

Action of $\Gamma_{0}$ on $L^{2}[0,1]$ is distance preserving, C4.1

Action of $\Gamma_{0}$ on $L^{2}[0,1]$ is norm preserving, C4.2

adapt-DP, O4.15

admissible class $\Gamma$ of warping functions, D4.2

a.e., D2.15

algebra, D2.1

almost everywhere, D2.15

Approximation of a measurable function by simple functions, P2.23

Banach-Zarecki Theorem, P3.22

Borel set, D2.3

Bounded Convergence Theorem, C2.6

bounded variation, D3.3

canonical representation, O2.11

Cantor function, O3.3

Cantor set, O2.4

Carathéodory's criterion, D2.9

Cauchy sequence, D2.23

Chain rule, P3.26

Chain rule (Alternate form), C3.13 
Change of variable for Lebesgue integral, P3.27

Change of variable for Lebesgue integral (Alternate form I), C3.14

Change of variable for Lebesgue integral (Alternate form II), C3.15

Change of variable for Lebesgue integral over a measurable set, C3.16

Change of variable for Riemann integral, P3.14

characteristic function, D2.13

closed set, D2.3

$\operatorname{cl}\left([q]_{\Gamma_{0}}\right), \mathrm{D} 4.6$

compact set, D2.4

complete normed linear space, D2.23

Composition of absolutely continuous functions, P3.25

Constant-speed parametrization of an absolutely continuous function, P4.14

continuous, O2.10

converge in norm, D2.23

Countable additivity of $m, \mathrm{P} 2.12$

Countable additivity of the Lebesgue integral, P2.36

Countable subadditivity of $m, \mathrm{P} 2.12$

Countable subadditivity of $m^{*}, \mathrm{P} 2.7$

Density of simple and step functions in $L^{p}$ space, P2.44

$d\left(\operatorname{cl}\left(\left[q_{1}\right]_{\Gamma_{0}}\right), \operatorname{cl}\left(\left[q_{2}\right]_{\Gamma_{0}}\right)\right), \mathrm{C} 4.3$

$d\left(\left[q_{1}\right]_{\Gamma_{0}},\left[q_{2}\right]_{\Gamma_{0}}\right), \mathrm{O} 4.12$

derivative, D3.1

diffeomorphism, O4.6

Differentiability of the indefinite integral, P3.7

Differentiability of the Riemann integral, C3.1

differentiable, D3.1

differentiable (generalized to smooth manifolds), O4.6

differentiable manifolds, O4.6

differential, O4.6

Distance between equivalence classes in $L^{2}[0,1] / \sim, \mathrm{C} 4.3$

Distance between functions in $A C[0,1], \mathrm{O} 4.11$

Egoroff's Theorem, P2.22

Equivalent conditions for a measurable function, P2.17

Equivalent conditions for a measurable set, P2.15

Equivalent conditions for an absolutely continuous function, P3.12

extended real numbers, D2.7

extended to all of $[c, d]$, O3.8

$f$ extended to all of $[c, d]$, O3.8 
Fatou's Lemma, P2.39

Fisher-Rao distance, O4.11

Fisher-Rao metric, D4.4

Fisher-Rao metric on $A C^{0}[0,1]$ under SRSF representation becomes $L^{2}[0,1]$ metric, P4.9

Fundamental Theorem of calculus I, P3.1

Fundamental Theorem of calculus II, P3.2

Fundamental Theorem of calculus for continuous functions, C3.1

Fundamental Theorem of Lebesgue integral calculus, C3.6

Fundamental Theorem of Lebesgue integral calculus (Alternate form), P3.13

$\Gamma, \mathrm{D} 4.2$

$\Gamma_{0}, \mathrm{D} 4.2$

geodesic, $\mathrm{O} 4.6$

geodesic distance, $\mathrm{O} 4.6$

group $\Gamma_{0}$ of invertible warping functions, D4.2

Heine-Borel, P2.3

Hölder's inequality, P2.41

indefinite integral, D3.4

Indefinite integral of $f$ zero everywhere, then $f$ is zero a.e., P3.6

Integrable equivalent to measurable, P2.32

Inverse function theorem, P3.23

invertible warping functions, D4.2

isometry, O4.6

Jordan decomposition, P3.5

$L^{2}[0,1] / \sim, \mathrm{O} 4.13$

$L^{2}[0,1] / \Gamma_{0}, \mathrm{O} 4.12$

$L^{2}[0,1]$ 's equivalence with the set of all SRSF's, P4.3

$L^{p}[0,1]$ or $L^{p}$ space, D2.22

$L^{p}$ norm, D2.22

$L^{p}$ norm of a function, D2.22

$L^{\infty}[0,1]$ or $L^{\infty}$ space, D2.22

$L^{\infty}$ norm, D2.22

$L^{\infty}$ norm of a function, D2.22

Lebesgue integrable, D2.21

Lebesgue integral of a measurable function, D2.21

Lebesgue integral of a measurable nonnegative function, D2.20

Lebesgue integral of a simple function, D2.19

(Lebesgue) measurable function, D2.11 
(Lebesgue) measurable set, D2.9

Lebesgue measure $m, \mathrm{D} 2.10$

Lebesgue's criterion for Riemann integrability, P2.35

Lebesgue's Dominated Convergence Theorem, P2.40

Lebesgue's Monotone Convergence Theorem, P2.37

left-hand limit, D3.2

limit point, D2.5

Lindelöf, P2.2

lower Riemann integral, D2.17

Lusin's Theorem, P2.24

$m, \mathrm{D} 2.10$

$m^{*}, \mathrm{D} 2.8$

Measurability of the derivative of a measurable function, P3.19

measurable function, D2.11

measurable set, D2.9

mesh, D2.18

Minkowski's inequality, P2.42

Monotonic functions: continuity, P3.3

Monotonic functions: differentiability, P3.4

Monotonic surjective $f$ implies $f$ is continuous, C3.2

negative part $f^{-}$of $f$, D2.16

Nested sequences of measurable sets Lemma, P2.13

normed linear space with norm $\|\cdot\|$, D2.23

open set, D2.3

optimal matching, O4.17

orbit of the constant function equal to 1, C4.4

orbit $[q]_{\Gamma}$ of $q$ under $\Gamma, \mathrm{D} 4.7$

orbit $[q]_{\Gamma_{0}}$ of $q$ under $\Gamma_{0}, \mathrm{D} 4.6$

outer measure $m^{*}$, D2.8

partition, D2.12

perfect set, D2.6

positive part $f^{+}$of $f, \mathrm{D} 2.16$

$[q]_{\Gamma}, \mathrm{D} 4.7$

$[q]_{\Gamma_{0}}, \mathrm{D} 4.6$

Reconstruction of an absolutely continuous function from its SRSF, P4.2

Riemannian manifolds, O4.6

Riemannian metric, O4.6

Riemannian structure, O4.6 
Riemann integrable, D2.17

Riemann integrable implies Lebesgue integrable, P2.33

Riemann integral, D2.17

Riemann sum, D2.18

Riemann sums of $f$ converge if and only $f$ is Riemann integrable, P2.27

Riemann sums of $f$ that converge implies $f$ is bounded, P2.26

Riesz-Fischer, P2.43

right-hand limit, D3.2

Saks' inequality, P3.17

Saks' Theorem, C3.10

Schwarz's inequality, O2.25

semigroup $\Gamma$ of warping functions, D4.2

semi-isometry, O4.6

Serrin-Varberg's Theorem, P3.18

Serrin-Varberg's Theorem (Alternate form), C3.12

$S F[0,1], \mathrm{D} 4.8$

$\sigma$-algebra, D2.2

simple function, D2.14

smooth manifolds, O4.6

Square integrability of SRSF, P4.1

square-root slope function (SRSF), D4.1

square-root velocity function (SRVF), O4.2

SRSF of a warped absolutely continuous function, P4.4

SRSF representation of functions, D4.1

SRSF's of functions in $\Gamma_{0}$ and $\Gamma, \mathrm{C} 4.4$

SRVF, O4.2

standard form, D4.8

step function, D2.12

subdivision, D2.12

Substitution rule for Lebesgue integral, P3.16

Substitution rule for Riemann integral, P3.15

$T_{p} M, 04.6$

tangent space $T_{p} M, \mathrm{O} 4.6$

uniformly continuous, O3.3

Uniqueness of constant-speed parametrization, C4.9

upper Riemann integral, D2.17

Zarecki's criterion for an absolutely continuous inverse, P3.24 


\section{Appendix: Change Log}

Revision 2 Release 2 - June 27, 2019

July 18, 2018 updates:

- Added one sentence for case $L=0$ in proof of Proposition 4.14.

- Added Observation 4.17.

June 27, 2019 updates:

- Added DOI that points to data in Observation 4.15. 